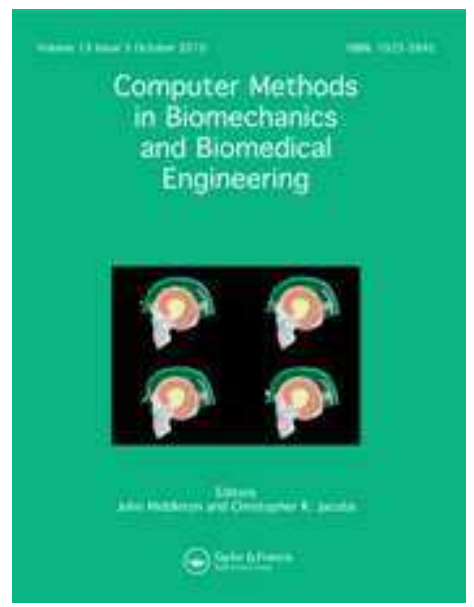

\title{
A Fast Strong Coupling Algorithm for the Partitioned Fluid- Structure Interaction Simulation of BMHVs
}

\begin{tabular}{|r|l|}
\hline Journal: & Computer Methods in Biomechanics and Biomedical Engineering \\
\hline Manuscript ID: & Draft \\
\hline Manuscript Type: & Original Article \\
\hline Author: & n/a \\
\hline Complete List of Authors: & $\begin{array}{l}\text { Annerel, Sebastiaan; Ghent University, Department of Flow, Heat } \\
\text { and Combustion Mechanics } \\
\text { Degroote, Joris; Ghent University, Department of Flow, Heat and } \\
\text { Combustion Mechanics } \\
\text { Claessens, Tom; University College Ghent, Department of } \\
\text { Mechanics } \\
\text { Dahl, Sigrid; Norwegian University of Science and Technology, } \\
\text { Department of Structural Engineering; Simula Research Laboratory } \\
\text { Skallerud, Bjørn; Norwegian University of Science and Technology, } \\
\text { Department of Structural Engineering } \\
\text { Hellevik, Leif Rune; Norwegian University of Science and } \\
\text { Technology, Department of Structural Engineering } \\
\text { Van Ransbeeck, Peter; University College Ghent, Department of } \\
\text { Mechanics } \\
\text { Segers, Patrick; Ghent University, IBiTech-bioMMeda } \\
\text { Verdonck, Pascal; Ghent University, IBiTech-bioMMeda } \\
\text { vierendeels, Jan; Ghent University, Department of Flow, Heat and } \\
\text { Combustion Mechanics }\end{array}$ \\
\hline Keywords: & $\begin{array}{l}\text { Fluid-Structure Interaction, BMHV, algorithm, quasi-Newton, } \\
\text { partitioned }\end{array}$ \\
\hline \hline
\end{tabular}




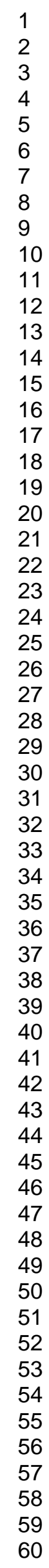

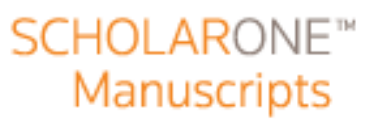

23

24

26

27

29

30 


\section{A Fast Strong Coupling Algorithm for the Partitioned Fluid- Structure Interaction Simulation of BMHVs}

Sebastiaan Annerel $^{\mathrm{a} *}$, Joris Degroote ${ }^{\mathrm{a}}$, Tom Claessens ${ }^{\mathrm{b}}$, Sigrid K. Dahl ${ }^{\mathrm{c}, \mathrm{d}}$, Bjørn Skallerud $^{\mathrm{c}}$, Leif Rune Hellevik ${ }^{\mathrm{c}}$, Peter Van Ransbeeck ${ }^{\mathrm{b}}$, Patrick Segers ${ }^{\mathrm{e}}$, Pascal Verdonck ${ }^{\mathrm{e}}$ and Jan Vierendeels ${ }^{\mathrm{a}}$

${ }^{a}$ Department of Flow, Heat and Combustion Mechanics, Ghent University, Ghent, Belgium;

${ }^{b}$ Department of Mechanics, University College Ghent, Ghent, Belgium;

${ }^{c}$ Department of Structural Engineering, Norwegian University of Science and Technology, Trondheim, Norway;

${ }^{d}$ Simula Research Laboratory, Oslo, Norway;

IBiTech-bioMMeda, Ghent University, Ghent, Belgium.

*Corresponding author. Email: Sebastiaan.Annerel@UGent.be 


\title{
A Fast Strong Coupling Algorithm for the Partitioned Fluid- Structure Interaction Simulation of BMHVs
}

\begin{abstract}
The numerical simulation of Bileaflet Mechanical Heart Valves (BMHVs) has gained strong interest in the last years, as a design and optimization tool. In this paper, a strong coupling algorithm for the partitioned fluid-structure interaction (FSI) simulation of a BMHV is presented. The convergence of the coupling iterations between the flow solver and the leaflet motion solver is accelerated by using the Jacobian with the derivatives of the pressure and viscous moments acting on the leaflets with respect to the leaflet accelerations. This Jacobian is numerically calculated from the coupling iterations. An error analysis is performed to derive a criterion for the selection of useable coupling iterations. The algorithm is successfully tested for two 3D cases of a BMHV and a comparison is made with existing coupling schemes. It is observed that the developed coupling scheme outperforms these existing schemes in needed coupling iterations per time step and CPU time.
\end{abstract}

Keywords: Fluid-Structure Interaction; BMHV; algorithm; quasi-Newton; partitioned

\section{Introduction}

Bileaflet Mechanical Heart Valves (BMHVs) are widely used and preferred for valve replacement because of their durability. However, current BMHVs induce calcification and thromboembolism among others, which is believed to be due to nonphysiological flow and turbulence generated by the valve leaflets. Therefore, numerical flow simulations can provide relevant information for valve design optimization (Sotiropoulous and Borazjani 2009). Such numerical simulation of a BMHV is a complex Fluid-Structure Interaction (FSI) problem, because the movement of the leaflets strongly interacts with the surrounding fluid motion and, therefore, the equilibrium at the fluid-structure interface needs to be taken into account. Several FSI methods are developed and used for the simulation of BMHVs. These methods can be classified in two ways.

A first classification concerns the kinematical description of the domain. For a structural problem, the motion is usually described by the Lagrangian method, where the computational grid moves with the material velocity. This is in contrast to a fluid domain, in which the motion is generally described by the Eulerian method and, therefore, the computational grid does not deform. In case of fluid-structure interaction, both methods can be combined in several ways in order to describe the motion of the domain.

One possibility is the "fixed grid" approach. In this method, an immersed structure is allowed to "fictitiously" move through the Eulerian fluid grid in a Lagrangian way. The influence of the structure boundary on the fluid outside the structure is calculated by the introduction of body force sources into the Navier-Stokes equations, while keeping the velocity of the fluid overlapped by the structure equal to the structural velocity. Since the fluid grid is fixed, there is no need for remeshing and grid adaption. However, because the fluid-structure interface is not necessarily aligned with the spatial discretization and the data are, as such, interpolated, the flow field (and thus shear stresses) at this interface is not accurately calculated. Several fixed grid methods were developed, like the Immersed Boundary (IB) method, as first 
proposed by Peskin (1972) for the simulation of heart valves. Borazjani et al. (2008) used the sharp interface CURVIB-method for the simulation of a BMHV. Other IB techniques were developed and used by Tai et al. (2007), De Tullio et al. (2009) and Xia et al. (2009). Also, the Fictitious Domain (FD) method can be employed to simulate flexible heart valves (Dos Santos et al. 2008; Astorino et al. 2009). This fixed grid method uses Lagrange multipliers to impose the kinematic constraints. Van Loon et al. (2004) improved the accuracy of the FD method by proposing a local fluid grid adaption at the structure boundary.

Another possibility is the use of the arbitrary Lagrangian-Eulerian (ALE) method for the fluid domain. In this "moving grid" method, the fluid grid motion is driven by the motion of its boundaries which are typically common boundaries of the moving fluid domain and the moving structure. The fluid grid velocity is called "arbitrary" since it does not correspond to the fluid velocity. However, when the grid deformation becomes too large, as is the case with BMHVs, this could deteriorate the grid quality. Therefore, local remeshing is needed between time steps. The main advantage of the ALE approach is its accuracy, since the grid is aligned with the fluid-structure interface. However, the use of remeshing (and thus interpolation) introduces artificial diffusivity and can become expensive for complex three-dimensional geometries. Several studies used the ALE approach to simulate the dynamics of the ATS Open Pivot $^{\mathrm{TM}}$ Standard Heart Valve (Dumont et al. 2005, 2007), the St. Jude Medical ${ }^{\mathrm{TM}}$ BMHV (Penrose et al. 2002; Redaelli et al. 2004; Dumont et al. 2007; Guivier et al. 2007, 2009; Nobili et al. 2007, 2008; Choi et al. 2009; Hong et al. 2009) and other valve types (Makhijani et al. 1997; Vierendeels et al. 2005, 2007; Bang et al. 2006; Morsi et al. 2007).

Secondly, one can classify each FSI simulation by the use of a partitioned solver or a monolithic solver. In the monolithic approach, the entire FSI problem is simultaneously simulated by one solver. This is in contrast to the partitioned approach, which solves the flow and the structural problem separately and, therefore, mostly uses different specialised solvers. The partitioned approach is used to simulate heart valves in Makhijani et al. (1997), Penrose et al. (2002), Redaelli et al. (2004), Dumont et al. (2005, 2007), Vierendeels et al. (2005, 2007), Bang et al. (2006), Guivier et al. (2007, 2009), Nobili et al. (2007, 2008), Morsi et al. (2007), Tai et al. (2007), Borazjani et al. (2008), Dos Santos et al. (2008), Astorino et al. (2009), Choi et al. (2009), De Tullio et al. (2009), Hong et al. (2009) and, finally, Xia et al. (2009). In order to obtain the interaction between the fluid and the structure, data exchange at the fluid-structure interface and a coupling scheme between the separated solvers are needed. Unfortunately, not every coupling scheme reaches convergence. The instability of coupling schemes without relaxation is analytically explained in (Vierendeels et al. 2005; Borazjani et al. 2008) for the case of BMHVs, and it is also demonstrated by the flow through arteries (Causin et al. 2005; Degroote et al. 2008, 2010). It is concluded that relaxation can be used to obtain stable and efficient approximations for the subsequent movements of the valve leaflets. Several coupling schemes with relaxation have thus been developed and they can be divided into loose and strong coupling schemes.

In the loose coupling methods, only one coupling iteration is needed in each time step, since the solution of the flow field at time step $n$ is used to calculate the angular accelerations of the leaflets for the next time step $n+1$. The loose coupling formulation is often used to simulate heart valves (Redaelli et al. 2004; Nobili et al. 2007; Morsi et al. 2007; Tai et al. 2007; Borazjani et al. 2008; Xia et al. 2009). Note that in these loose coupling formulations a relaxation factor is used for reasons of stability, as 
discussed above. The loose coupling method has the main benefit of the low computational cost due to the lack of a coupling iteration loop within each time step. However, this lack implies that the equilibrium of Newton's Second Law at the leaflet walls is not necessarily achieved.

Such dynamic equilibrium at the fluid-structure interface can be obtained by introducing a coupling iteration loop within each time step, as is the case in the strong coupling methods. However, the introduction of a coupling loop requires a stable and efficient approximation of the subsequent leaflet angular accelerations in each coupling iteration, which can be done for example by using fixed point iterations with relaxation. For the simulation of a BMHV in a partitioned way, several methods can be used to calculate an appropriate relaxation factor. In the following, the use of a fixed relaxation factor and a dynamic relaxation factor is discussed.

For the fixed relaxation factor, the factor value is kept constant during the entire time cycle, as described in Le Tallec and Mouro (2001) for an industrial shock absorber valve. Fixed relaxation was used to simulate the dynamics of a BMHV with a partitioned solver by Penrose et al. (2002), Nobili et al. (2007, 2008), Borazjani et al. (2008), De Tullio et al. (2009) and Hong et al. (2009). The main disadvantage of such a fixed relaxation is the lack of a physical meaning of the relaxation factor. Therefore, the selection of an appropriate factor value is ad hoc and will mostly be done through trial-and-error, as noted by De Tullio et al. (2009).

For the dynamic relaxation factor, the factor value is updated in each coupling iteration of each time step. Typically, the Aitken $\Delta^{2}$ relaxation is used, as described in Irons et al. (1969), Mok et al. (2001) and Küttler et al. (2008). Partitioned simulations of heart valves using the Aitken $\Delta^{2}$ relaxation method are found in Borazjani et al. (2008), Dos Santos et al. (2008) and Astorino et al. (2009).

In this paper, a strong coupling algorithm for the simulation of a BMHV by a partitioned solver with a dynamic relaxation matrix is presented and evaluated. The algorithm predicts the leaflet moments (and thus the leaflet angular accelerations) of the next coupling iteration through a linearization of Newton's Second Law with a finite difference approximation of the Jacobian. The components of this Jacobian are the derivatives of the moments (exerted by the flow on the leaflets) with respect to the leaflet angular accelerations. The Jacobian is numerically calculated from the flow solver by variations of the leaflet positions. This quasi-Newton method was first introduced in Vierendeels et al. (2005) for one stiff leaflet and subsequently used to simulate a BMHV (Dumont et al. 2005, 2007) and later adopted by Guivier et al. (2007, 2009). However, the algorithm described in Vierendeels et al. (2005) had only one degree of freedom which implied a symmetrical motion of both leaflets when simulating a BMHV. Therefore, the algorithm was extended to two degrees of freedom in Annerel et al. (2010) and Dahl et al. (2010), thus allowing the two leaflets to move asynchronously. In this paper, the convergence process is accelerated by the use of a variable time step size, extrapolation techniques and reuse of data from previous time steps. Also, the calculation process of the Jacobian is made more efficient, thus resulting in faster convergence.

The paper is organized as follows. First, the algorithm is derived and its implementation in a commercially available black box flow solver which uses the ALE approach, is analysed. Secondly, the algorithm is tested by two 3D cases of a BMHV. One case consists of a rigid straight tube. The second case is made asymmetrical by placing Valsalva sinuses downstream of the valve. Subsequently, the characteristics of the previously mentioned existing coupling schemes are discussed and their stability and efficiency is compared through numerical experiments for the 
3D BMHV cases. Finally, it is concluded that the developed FSI algorithm for the partitioned simulation of a BMHV converges quickly and outperforms the existing coupling schemes in needed coupling iterations per time step and CPU time.

\section{Methods}

\subsection{Governing equations}

A BMHV can be modelled as a rigid casing in which two separate rigid leaflets rotate around their hinge axes. Because the position of each rigid leaflet is solely determined by its opening angle, the bileaflet valve has two degrees of freedom.

The movement of a rigid leaflet is governed by Newton's Second Law, which states that the moment about its hinge must be in equilibrium with the product of its moment of inertia and its angular acceleration. For two leaflets, this gives the following two equations with $M_{i}, I_{i}$ and $\ddot{\theta}_{i}$ representing, respectively, the moment, the moment of inertia and the angular acceleration of leaflet $i$ about its rotation axis:

$$
\left\{\begin{array}{l}
M_{1}=I_{1} \cdot \ddot{\theta}_{1} \\
M_{2}=I_{2} \cdot \ddot{\theta}_{2}
\end{array}\right.
$$

When the hinges are modelled as frictionless, the moment acting on the leaflet is the pressure and viscous moment exerted by the flow.

\subsection{Existing methods}

In the loose coupling methods, as used by Redaelli et al. (2004), Morsi et al. (2007), Nobili et al. (2007), Tai et al. (2007), Borazjani et al. (2008) and Xia et al. (2009), the solution from the flow field at time step $n$ is used to calculate the angular accelerations for the next time step $n+1$ :

$$
\ddot{\theta}_{i}^{n+1}=\left(1-\omega_{i}^{n+1}\right) \cdot \ddot{\theta}_{i}^{n}+\omega_{i}^{n+1} \cdot \frac{M_{i}^{n}}{I_{i}}
$$

A relaxation factor $\left(\omega_{i}{ }^{n+1}<1\right)$ is necessary, since the scheme without relaxation $\left(\omega_{i}{ }^{n+1}=1\right)$ is unstable (Causin et al. 2005; Vierendeels et al. 2005; Borazjani et al. 2008).

Strong coupling schemes achieve dynamic equilibrium at the fluid-structure interface by introducing a coupling iteration loop within each time step, as visualized in Figure 1. Generally, each of the coupling iterations follows the same pattern. At the beginning of each coupling iteration $k$ of time step $n+1$, the motion of the leaflets is computed from the angular accelerations $\ddot{\theta}_{i}^{n+1, k}$. The mesh is moved and the NavierStokes equations are solved. From the flow field, the moments $M_{i}^{n+1, k}$ are calculated. Finally, the convergence of the dynamic equilibrium at the fluid-structure interface (i.e. the "FSI convergence", expressed by Equation (1)) is checked. When this FSI convergence is obtained, a new time step is initiated. However, when the FSI convergence is not achieved, a new coupling iteration $k+1$ is initiated and thus new angular accelerations $\ddot{\theta}_{i}^{n+1, k+1}$ need to be calculated. Therefore, the introduction of a coupling iteration loop requires, in each coupling iteration $k$ of time step $n+1$, a stable and efficient approximation of the angular accelerations for the next coupling iteration $k+1$. 
A strong coupling scheme without relaxation can easily be proposed. From the moments of coupling iteration $k$ in time step $n+1$, the angular accelerations of the next coupling iteration $k+1$, i.e. $\ddot{\theta}_{i}^{n+1, k+1}$, can be calculated:

$$
\ddot{\theta}_{i}^{n+1, k+1}=\frac{M_{i}^{n+1, k}}{I_{i}}
$$

Unfortunately, such fixed point iterations, also called Gauss-Seidel iterations without relaxation, are unstable for BMHVs (Vierendeels et al. 2005). Therefore, the scheme needs to be stabilized by the use of an appropriate prediction of the moments of the next coupling iteration $k+1$ :

$$
\ddot{\theta}_{i}^{n+1, k+1}=\frac{\hat{M}_{i}^{n+1, k+1}}{I_{i}}
$$

with $\hat{M}_{i}^{n+1, k+1}$ denoting the predicted moment. Several methods are used in literature to calculate a stable value for $\hat{M}_{i}^{n+1, k+1}$. Usually, this is achieved by a relaxation scheme, leading to fixed point iterations with relaxation $\left(\omega_{i}^{n+1, k}<1\right)$, also called the Gauss-Seidel coupling method with relaxation:

$$
\hat{M}_{i}^{n+1, k+1}=\left(1-\omega_{i}^{n+1, k}\right) \cdot I_{i} \ddot{\theta}_{i}^{n+1, k}+\omega_{i}^{n+1, k} \cdot M_{i}^{n+1, k}
$$

Inserted in Equation (4):

$$
\ddot{\theta}_{i}^{n+1, k+1}=\left(1-\omega_{i}^{n+1, k}\right) \cdot \ddot{\theta}_{i}^{n+1, k}+\omega_{i}^{n+1, k} \cdot \frac{M_{i}^{n+1, k}}{I_{i}}
$$

As mentioned in Section 1, several methods can be used to calculate an appropriate relaxation factor $\omega_{i}{ }^{n+1, k}$ that stabilizes the solution process. For example, the relaxation factor can be chosen as fixed during the entire simulation (Le Tallec and Mouro 2001; Penrose et al. 2002; Nobili et al. 2007, 2008; Borazjani et al. 2008; De Tullio et al. 2009; Hong et al. 2009):

$$
\omega_{i}^{n+1, k}=\omega=C s t
$$

Furthermore, a dynamic Aitken $\Delta^{2}$ relaxation factor can be used (Irons et al. 1969; Mok et al. 2001; Küttler et al. 2008). The Aitken relaxation updates the value of the factor in each coupling iteration $k>0$ of each time step $n+1$ :

with

$$
\omega_{i}^{n+1, k}=\omega^{n+1, k}=-\frac{\left(\ddot{\boldsymbol{\theta}}^{n+1, k}-\ddot{\boldsymbol{\theta}}^{n+1, k-1}\right)^{T}\left(\boldsymbol{r}^{n+1, k}-\boldsymbol{r}^{n+1, k-1}\right)}{\left(\boldsymbol{r}^{n+1, k}-\boldsymbol{r}^{n+1, k-1}\right)^{T}\left(\boldsymbol{r}^{n+1, k}-\boldsymbol{r}^{n+1, k-1}\right)}
$$

$$
\ddot{\boldsymbol{\theta}}^{n+1, k}=\left[\begin{array}{l}
\ddot{\theta}_{1}^{n+1, k} \\
\ddot{\theta}_{2}^{n+1, k}
\end{array}\right] \text { and } \boldsymbol{r}^{n+1, k}=\left[\begin{array}{ll}
\frac{M_{1}^{n+1, k}}{I_{1}}-\ddot{\theta}_{1}^{n+1, k} \\
\frac{M_{2}^{n+1, k}}{I_{2}}-\ddot{\theta}_{2}^{n+1, k}
\end{array}\right]
$$

\subsection{Quasi-Newton method}

A stable prediction of $\hat{M}_{i}^{n+1, k+1}$ in Equation (4) can also be achieved through a linearization of Newton's Second Law, as analysed by Vierendeels et al. (2005) for a monoleaflet heart valve. Thus, while taking into account the mutual interaction between the leaflets, Equation (1) is linearized for each coupling iteration $k+1$ of time step $n+1$ : 


$$
\left\{\begin{array}{l}
M_{1}^{n+1, k}+\left(\frac{\partial M_{1}}{\partial \ddot{\theta}_{1}}\right)^{n+1, k}\left(\ddot{\theta}_{1}^{n+1, k+1}-\ddot{\theta}_{1}^{n+1, k}\right)+\left(\frac{\partial M_{1}}{\partial \ddot{\theta}_{2}}\right)^{n+1, k}\left(\ddot{\theta}_{2}^{n+1, k+1}-\ddot{\theta}_{2}^{n+1, k}\right)=I_{1} \cdot \ddot{\theta}_{1}^{n+1, k+1} \\
M_{2}^{n+1, k}+\left(\frac{\partial M_{2}}{\partial \ddot{\theta}_{1}}\right)^{n+1, k}\left(\ddot{\theta}_{1}^{n+1, k+1}-\ddot{\theta}_{1}^{n+1, k}\right)+\left(\frac{\partial M_{2}}{\partial \ddot{\theta}_{2}}\right)^{n+1, k}\left(\ddot{\theta}_{2}^{n+1, k+1}-\ddot{\theta}_{2}^{n+1, k}\right)=I_{2} \cdot \ddot{\theta}_{2}^{n+1, k+1}
\end{array}\right.
$$

The derivatives $\frac{\partial M_{i}}{\partial \ddot{\theta}_{j}}$ will be discussed further on.

Equation (10) can be rearranged as follows:

$$
\left\{\begin{array}{l}
I_{1} \cdot \ddot{\theta}_{1}^{n+1, k+1}-\left(\frac{\partial M_{1}}{\partial \ddot{\theta}_{1}}\right)^{n+1, k} \ddot{\theta}_{1}^{n+1, k+1}-\left(\frac{\partial M_{1}}{\partial \ddot{\theta}_{2}}\right)^{n+1, k} \ddot{\theta}_{2}^{n+1, k+1}=M_{1}^{n+1, k}-\left(\frac{\partial M_{1}}{\partial \ddot{\theta}_{1}}\right)^{n+1, k} \ddot{\theta}_{1}^{n+1, k}-\left(\frac{\partial M_{1}}{\partial \ddot{\theta}_{2}}\right)^{n+1, k} \ddot{\theta}_{2}^{n+1, k} \\
I_{2} \cdot \ddot{\theta}_{2}^{n+1, k+1}-\left(\frac{\partial M_{2}}{\partial \ddot{\theta}_{1}}\right)^{n+1, k} \ddot{\theta}_{1}^{n+1, k+1}-\left(\frac{\partial M_{2}}{\partial \ddot{\theta}_{2}}\right)^{n+1, k} \ddot{\theta}_{2}^{n+1, k+1}=M_{2}^{n+1, k}-\left(\frac{\partial M_{2}}{\partial \ddot{\theta}_{1}}\right)^{n+1, k} \ddot{\theta}_{1}^{n+1, k}-\left(\frac{\partial M_{2}}{\partial \ddot{\theta}_{2}}\right)^{n+1, k} \ddot{\theta}_{2}^{n+1, k}
\end{array}\right.
$$

This can be written in matrix notation:

$$
\left[\begin{array}{cc}
I_{1}-\left(\frac{\partial M_{1}}{\partial \ddot{\theta}_{1}}\right)^{n+1, k} & -\left(\frac{\partial M_{1}}{\partial \ddot{\theta}_{2}}\right)^{n+1, k} \\
-\left(\frac{\partial M_{2}}{\partial \ddot{\theta}_{1}}\right)^{n+1, k} & I_{2}-\left(\frac{\partial M_{2}}{\partial \ddot{\theta}_{2}}\right)^{n+1, k}
\end{array}\right] \cdot\left[\begin{array}{l}
\ddot{\theta}_{1}^{n+1, k+1} \\
\ddot{\theta}_{2}^{n+1, k+1}
\end{array}\right]=\left[\begin{array}{l}
M_{1}^{n+1, k}-\left(\frac{\partial M_{1}}{\partial \ddot{\theta}_{1}}\right)^{n+1, k} \ddot{\theta}_{1}^{n+1, k}-\left(\frac{\partial M_{1}}{\partial \ddot{\theta}_{2}}\right)^{n+1, k} \ddot{\theta}_{2}^{n+1, k} \\
M_{2}^{n+1, k}-\left(\frac{\partial M_{2}}{\partial \ddot{\theta}_{1}}\right)^{n+1, k} \ddot{\theta}_{1}^{n+1, k}-\left(\frac{\partial M_{2}}{\partial \ddot{\theta}_{2}}\right)^{n+1, k} \ddot{\theta}_{2}^{n+1, k}
\end{array}\right]
$$

With a change in notation, leading to:

wherein

$$
\left[\boldsymbol{I}_{12}-\boldsymbol{J}^{n+1, k}\right] \cdot \ddot{\boldsymbol{\theta}}^{n+1, k+1}=\boldsymbol{M}^{n+1, k}-\boldsymbol{J}^{n+1, k} \cdot \ddot{\boldsymbol{\theta}}^{n+1, k}
$$

$$
\boldsymbol{I}_{12}=\left[\begin{array}{cc}
I_{1} & 0 \\
0 & I_{2}
\end{array}\right], \boldsymbol{J}^{n+1, k}=\left[\begin{array}{ll}
\left(\frac{\partial M_{1}}{\partial \ddot{\theta}_{1}}\right)^{n+1, k} & \left(\frac{\partial M_{1}}{\partial \ddot{\theta}_{2}}\right)^{n+1, k} \\
\left(\frac{\partial M_{2}}{\partial \ddot{\theta}_{1}}\right)^{n+1, k} & \left(\frac{\partial M_{2}}{\partial \ddot{\theta}_{2}}\right)^{n+1, k}
\end{array}\right], \text { and } \boldsymbol{M}^{n+1, k}=\left[\begin{array}{c}
M_{1}^{n+1, k} \\
M_{2}^{n+1, k}
\end{array}\right]
$$

Equation (13) can be simplified in notation as

with

$$
\boldsymbol{A}^{n+1, k} \cdot \ddot{\boldsymbol{\theta}}^{n+1, k+1}=\boldsymbol{B}^{n+1, k}
$$

$$
\begin{gathered}
\boldsymbol{A}^{n+1, k}=\boldsymbol{I}_{12}-\boldsymbol{J}^{n+1, k} \\
\boldsymbol{B}^{n+1, k}=\boldsymbol{M}^{n+1, k}-\boldsymbol{J}^{n+1, k} \cdot \ddot{\boldsymbol{\theta}}^{n+1, k}
\end{gathered}
$$

Thus, the column-matrix $\ddot{\boldsymbol{\theta}}^{n+1, k+1}$ contains the angular accelerations of coupling iteration $k+1$ at time step $n+1$. The matrix $\boldsymbol{A}^{n+1, k}$ is composed of $\boldsymbol{I}_{12}$ (i.e. the moments of inertia) and $\boldsymbol{J}^{n+1, k}$ (i.e. the Jacobian of the moments with respect to the angular accelerations, further referred to as "the Jacobian"). Finally, the column-matrix $\boldsymbol{B}^{n+1, k}$ consists of all the remaining factors of coupling iteration $k$ at time step $n+1$. For a well-posed problem, the matrix $\boldsymbol{A}^{n+1, k}$ has to be nonsingular. In that case, Equation (15) can be rewritten as

$$
\ddot{\boldsymbol{\theta}}^{n+1, k+1}=\left[\boldsymbol{A}^{n+1, k}\right]^{-1} \cdot \boldsymbol{B}^{n+1, k}
$$


It is noted that the above described approach, can also be written as a dynamic relaxation scheme, since previous equation can be rearranged as

$$
\ddot{\boldsymbol{\theta}}^{n+1, k+1}=\left[\boldsymbol{I}_{12}-\boldsymbol{J}^{n+1, k}\right]^{-1} \cdot\left[-\boldsymbol{J}^{n+1, k}\right] \cdot \ddot{\boldsymbol{\theta}}^{n+1, k}+\left[\boldsymbol{I}_{12}-\boldsymbol{J}^{n+1, k}\right]^{-1} \cdot \boldsymbol{M}^{n+1, k}
$$

or

with

$$
\ddot{\boldsymbol{\theta}}^{n+1, k+1}=\left[\boldsymbol{I}-\boldsymbol{\Omega}^{n+1, k}\right] \cdot \ddot{\boldsymbol{\theta}}^{n+1, k}+\boldsymbol{\Omega}^{n+1, k} \cdot \boldsymbol{I}_{12}^{-1} \cdot \boldsymbol{M}^{n+1, k}
$$

$$
\boldsymbol{\Omega}^{n+1, k}=\left[\boldsymbol{I}_{12}-\boldsymbol{J}^{n+1, k}\right]^{-1} \cdot \boldsymbol{I}_{12} \text { and } \boldsymbol{I}=\left[\begin{array}{ll}
1 & 0 \\
0 & 1
\end{array}\right]
$$

The relaxation is visible with $\boldsymbol{\Omega}^{n+1, k}$ as the relaxation matrix. Equation (18) is thus similar to Equation (6), but with matrix relaxation instead of a scalar relaxation factor.

In order to solve Equation (17) and thus to calculate the angular accelerations of the new coupling iteration $k+1$, the Jacobian has to be known. However, when a black box flow solver is used, these derivatives are not accessible. Fortunately, they can be approximated by finite differences $(i, j=1,2)$ :

$$
\frac{\partial M_{i}}{\partial \ddot{\theta}_{j}} \approx \frac{\Delta M_{i}}{\Delta \ddot{\theta}_{j}}
$$

These finite differences are calculated by solving the following system of equations:

$$
\left\{\begin{array}{l}
\left(\frac{\Delta M_{1}}{\Delta \ddot{\theta}_{1}}\right) \cdot \Delta \ddot{\theta}_{1}^{a}+\left(\frac{\Delta M_{1}}{\Delta \ddot{\theta}_{2}}\right) \cdot \Delta \ddot{\theta}_{2}^{a}=\Delta M_{1}^{a} \\
\left(\frac{\Delta M_{2}}{\Delta \ddot{\theta}_{1}}\right) \cdot \Delta \ddot{\theta}_{1}^{a}+\left(\frac{\Delta M_{2}}{\Delta \ddot{\theta}_{2}}\right) \cdot \Delta \ddot{\theta}_{2}^{a}=\Delta M_{2}^{a} \\
\left(\frac{\Delta M_{1}}{\Delta \ddot{\theta}_{1}}\right) \cdot \Delta \ddot{\theta}_{1}^{b}+\left(\frac{\Delta M_{1}}{\Delta \ddot{\theta}_{2}}\right) \cdot \Delta \ddot{\theta}_{2}^{b}=\Delta M_{1}^{b} \\
\left(\frac{\Delta M_{2}}{\Delta \ddot{\theta}_{1}}\right) \cdot \Delta \ddot{\theta}_{1}^{b}+\left(\frac{\Delta M_{2}}{\Delta \ddot{\theta}_{2}}\right) \cdot \Delta \ddot{\theta}_{2}^{b}=\Delta M_{2}^{b}
\end{array}\right.
$$

which results in

$$
\left\{\begin{array}{l}
\left(\frac{\Delta M_{1}}{\Delta \ddot{\theta}_{1}}\right)=\frac{\Delta M_{1}^{a} \cdot \Delta \ddot{\theta}_{2}^{b}-\Delta M_{1}^{b} \cdot \Delta \ddot{\theta}_{2}^{a}}{\Delta \ddot{\theta}_{1}^{a} \cdot \Delta \ddot{\theta}_{2}^{b}-\Delta \ddot{\theta}_{1}^{b} \cdot \Delta \ddot{\theta}_{2}^{a}} \\
\left(\frac{\Delta M_{1}}{\Delta \ddot{\theta}_{2}}\right)=\frac{\Delta M_{1}^{b} \cdot \Delta \ddot{\theta}_{1}^{a}-\Delta M_{1}^{a} \cdot \Delta \ddot{\theta}_{1}^{b}}{\Delta \ddot{\theta}_{1}^{a} \cdot \Delta \ddot{\theta}_{2}^{b}-\Delta \ddot{\theta}_{1}^{b} \cdot \Delta \ddot{\theta}_{2}^{a}} \\
\left(\frac{\Delta M_{2}}{\Delta \ddot{\theta}_{1}}\right)=\frac{\Delta M_{2}^{a} \cdot \Delta \ddot{\theta}_{2}^{b}-\Delta M_{2}^{b} \cdot \Delta \ddot{\theta}_{2}^{a}}{\Delta \ddot{\theta}_{1}^{a} \cdot \Delta \ddot{\theta}_{2}^{b}-\Delta \ddot{\theta}_{1}^{b} \cdot \Delta \ddot{\theta}_{2}^{a}} \\
\left(\frac{\Delta M_{2}}{\Delta \ddot{\theta}_{2}}\right)=\frac{\Delta M_{2}^{b} \cdot \Delta \ddot{\theta}_{1}^{a}-\Delta M_{2}^{a} \cdot \Delta \ddot{\theta}_{1}^{b}}{\Delta \ddot{\theta}_{1}^{a} \cdot \Delta \ddot{\theta}_{2}^{b}-\Delta \ddot{\theta}_{1}^{b} \cdot \Delta \ddot{\theta}_{2}^{a}}
\end{array}\right.
$$

The superscripts $a$ and $b$ refer to data obtained from three coupling iterations between which the leaflet angular accelerations of two coupling iterations have differences (according to the vectors $\Delta \ddot{\boldsymbol{\theta}}^{a}$ and $\boldsymbol{\Delta} \ddot{\boldsymbol{\theta}}^{b}$ ) with respect to a reference coupling iteration: 


$$
\begin{gathered}
\Delta \ddot{\boldsymbol{\theta}}^{a}=\ddot{\boldsymbol{\theta}}^{a}-\ddot{\boldsymbol{\theta}}^{r e f}=\left[\begin{array}{c}
\ddot{\theta}_{1}^{a}-\ddot{\theta}_{1}^{r e f} \\
\ddot{\theta}_{2}^{a}-\ddot{\theta}_{2}^{r e f}
\end{array}\right]=\left[\begin{array}{c}
\Delta \ddot{\theta}_{1}^{a} \\
\Delta \ddot{\theta}_{2}^{a}
\end{array}\right] \\
\Delta \ddot{\boldsymbol{\theta}}^{b}=\ddot{\boldsymbol{\theta}}^{b}-\ddot{\boldsymbol{\theta}}^{r e f}=\left[\begin{array}{c}
\ddot{\theta}_{1}^{b}-\ddot{\theta}_{1}^{r e f} \\
\ddot{\theta}_{2}^{b}-\ddot{\theta}_{2}^{r e f}
\end{array}\right]=\left[\begin{array}{c}
\Delta \ddot{\theta}_{1}^{b} \\
\Delta \ddot{\theta}_{2}^{b}
\end{array}\right]
\end{gathered}
$$

These differences in angular accelerations $\left(\Delta \ddot{\boldsymbol{\theta}}^{a}\right.$ and $\left.\Delta \ddot{\boldsymbol{\theta}}^{b}\right)$ induce differences in the calculated flow fields and thus also in the calculated moments, respectively denoted by $\Delta \boldsymbol{M}^{a}$ and $\boldsymbol{\Delta} \boldsymbol{M}^{b}$ :

$$
\begin{aligned}
& \Delta \boldsymbol{M}^{a}=\boldsymbol{M}^{a}-\boldsymbol{M}^{r e f}=\left[\begin{array}{l}
M_{1}^{a}-M_{1}^{r e f} \\
M_{2}^{a}-M_{2}^{r e f}
\end{array}\right]=\left[\begin{array}{c}
\Delta M_{1}^{a} \\
\Delta M_{2}^{a}
\end{array}\right] \\
& \Delta \boldsymbol{M}^{b}=\boldsymbol{M}^{b}-\boldsymbol{M}^{r e f}=\left[\begin{array}{l}
M_{1}^{b}-M_{1}^{r e f} \\
M_{2}^{b}-M_{2}^{r e f}
\end{array}\right]=\left[\begin{array}{l}
\Delta M_{1}^{b} \\
\Delta M_{2}^{b}
\end{array}\right]
\end{aligned}
$$

The data $\boldsymbol{\Delta} \ddot{\boldsymbol{\theta}}^{a}, \boldsymbol{\Delta} \ddot{\boldsymbol{\theta}}^{b}, \boldsymbol{\Delta} \boldsymbol{M}^{a}$ and $\boldsymbol{\Delta} \boldsymbol{M}^{\boldsymbol{b}}$ can be obtained from three coupling iterations by several methods.

\subsubsection{Calculate the Jacobian by existing method}

A first method to obtain the necessary data $\left(\boldsymbol{\Delta} \ddot{\boldsymbol{\theta}}^{a}, \boldsymbol{\Delta} \ddot{\boldsymbol{\theta}}^{\boldsymbol{b}}, \boldsymbol{\Delta} \boldsymbol{M}^{\boldsymbol{a}}\right.$ and $\left.\boldsymbol{\Delta} \boldsymbol{M}^{\boldsymbol{b}}\right)$ is described in Annerel et al. (2010) and Dahl et al. (2010) and uses three subsequent coupling iterations to compute the Jacobian. The coupling iteration $k$, used to determine the reference values $\left(\ddot{\boldsymbol{\theta}}^{\text {ref }}\right.$ and $\boldsymbol{M}^{\text {ref }}$ ), is denoted as $k^{r e f}$. In the two subsequent coupling iterations $\left(k=k^{r e f}+1\right.$ and $\left.k=k^{r e f}+2\right)$, a small perturbation $\delta$ is given to the angular acceleration of each of the leaflets, while keeping the other one fixed, as is described in Annerel et al. (2010) and Dahl et al. (2010). The value of this perturbation parameter $\delta$ may range between a minimal value (in order to obtain a significant difference between the "reference" moment and the "perturbation" moment) and a maximal value (above which the mesh motion could fail). In Vierendeels et al. (2005), it was shown that the quality of the estimation of the Jacobian is not very sensitive to the exact choice of this value.

In each coupling iteration, the flow field is solved from which, subsequently, the moments acting on the leaflets are calculated and stored. This can be summarized as follows:

$$
\begin{aligned}
& k^{r e f} \Rightarrow\left\{\begin{array} { l } 
{ \ddot { \theta } _ { 1 } ^ { n + 1 , k ^ { r e f } } } \\
{ \ddot { \theta } _ { 2 } ^ { n + 1 , k ^ { r e f } } }
\end{array} \quad \Rightarrow \left\{\begin{array}{l}
M_{1}^{n+1, k^{r e f}} \\
M_{2}^{n+1, k^{r e f}}
\end{array}\right.\right. \\
& k^{r e f}+1 \Rightarrow\left\{\begin{array} { l } 
{ \ddot { \theta } _ { 1 } ^ { n + 1 , k ^ { r e f } + 1 } = \ddot { \theta } _ { 1 } ^ { n + 1 , k ^ { r e f } } + \delta } \\
{ \ddot { \theta } _ { 2 } ^ { n + 1 , k ^ { r e f } + 1 } = \ddot { \theta } _ { 2 } ^ { n + 1 , k ^ { r e f } } }
\end{array} \Rightarrow \left\{\begin{array}{l}
M_{1}^{n+1, k^{r e f}+1} \\
M_{2}^{n+1, k^{r e f}+1}
\end{array}\right.\right. \\
& k^{r e f}+2 \Rightarrow\left\{\begin{array} { l } 
{ \ddot { \theta } _ { 1 } ^ { n + 1 , k ^ { r e f } + 2 } = \ddot { \theta } _ { 1 } ^ { n + 1 , k ^ { r e f } } } \\
{ \ddot { \theta } _ { 2 } ^ { n + 1 , k ^ { r e f } + 2 } = \ddot { \theta } _ { 2 } ^ { n + 1 , k ^ { \text { ref } } } + \delta }
\end{array} \Rightarrow \left\{\begin{array}{l}
M_{1}^{n+1, k^{\text {ref }}+2} \\
M_{2}^{n+1, k^{r e f}+2}
\end{array}\right.\right.
\end{aligned}
$$

This results in the following data: 


$$
\begin{aligned}
& \left\{\begin{array} { l } 
{ \Delta \ddot { \theta } _ { 1 } ^ { a } = \ddot { \theta } _ { 1 } ^ { n + 1 , k ^ { \text { ref } } + 1 } - \ddot { \theta } _ { 1 } ^ { n + 1 , k ^ { \text { ref } } } = \delta } \\
{ \Delta \ddot { \theta } _ { 2 } ^ { a } = \ddot { \theta } _ { 2 } ^ { n + 1 , k ^ { \text { ref } } + 1 } - \ddot { \theta } _ { 2 } ^ { n + 1 , k ^ { \text { ref } } = 0 } }
\end{array} \Rightarrow \left\{\begin{array}{l}
\Delta M_{1}^{a}=M_{1}^{n+1, k^{\text {ref }}+1}-M_{1}^{n+1, k^{\text {ref }}} \\
\Delta M_{2}^{a}=M_{2}^{n+1, k^{\text {ref }}+1}-M_{2}^{n+1, k^{\text {ref }}}
\end{array}\right.\right. \\
& \left\{\begin{array} { l } 
{ \Delta \ddot { \theta } _ { 1 } ^ { b } = \ddot { \theta } _ { 1 } ^ { n + 1 , k ^ { \text { ref } } + 2 } - \ddot { \theta } _ { 1 } ^ { n + 1 , k ^ { \text { ref } } } = 0 } \\
{ \Delta \ddot { \theta } _ { 2 } ^ { b } = \ddot { \theta } _ { 2 } ^ { n + 1 , k ^ { \text { ref } } + 2 } - \ddot { \theta } _ { 2 } ^ { n + 1 , k ^ { \text { ref } } } = \delta }
\end{array} \Rightarrow \left\{\begin{array}{l}
\Delta M_{1}^{b}=M_{1}^{n+1, k^{\text {ref }}+2}-M_{1}^{n+1, k^{\text {ref }}} \\
\Delta M_{2}^{b}=M_{2}^{n+1, k^{\text {ref }}+2}-M_{2}^{n+1, k^{\text {ref }}}
\end{array}\right.\right.
\end{aligned}
$$

of which the Jacobian components can be calculated by Equation (23).

\subsubsection{A new method for the Jacobian update}

In the previous method, three subsequent coupling iterations are needed of which the leaflet acceleration perturbation vectors $\Delta \ddot{\boldsymbol{\theta}}^{a}$ and $\Delta \ddot{\boldsymbol{\theta}}^{b}$ are perpendicular to each other and significantly large.

However, it is not necessary that the acceleration perturbation vectors $\Delta \ddot{\boldsymbol{\theta}}^{a}$ and $\Delta \ddot{\boldsymbol{\theta}}^{b}$ are completely perpendicular to each other. To calculate a good estimation of the Jacobian, it is already sufficient that their perpendicular components are significantly large. Moreover, the three coupling iterations do not have to be subsequent. Therefore, the calculation of the Jacobian can be made more efficient by the use of a criterion that selects two appropriate acceleration perturbation vectors (e.g. from previous coupling iterations), without the need for extra coupling iterations with perpendicularly perturbed accelerations. Thus, such a selection criterion needs to check the direction and the magnitude of the two acceleration perturbation vectors and is derived through an error propagation study in Appendix 1:

$$
\frac{\max _{i=1,2}\left(\sqrt{\left(\Delta \ddot{\theta}_{i}^{a}\right)^{2}+\left(\Delta \ddot{\theta}_{i}^{b}\right)^{2}}\right)}{\left|\Delta \ddot{\theta}_{2}^{b} \cdot \Delta \ddot{\theta}_{1}^{a}-\Delta \ddot{\theta}_{2}^{a} \cdot \Delta \ddot{\theta}_{1}^{b}\right|} \cdot \frac{\varepsilon_{1}}{\min _{i=1,2}\left(I_{i}\right)} \leq \varepsilon_{2}
$$

with the Jacobian update threshold $\varepsilon_{2}$ and the flow solver convergence threshold $\varepsilon_{1}$, which is the accuracy at which the moments are calculated in the flow solver. The appropriate values for $\varepsilon_{1}$ and $\varepsilon_{2}$ are determined by a sensitivity analysis, as is done in Section 3.4.1.

The above described criterion is used in an algorithm that makes it possible to update the Jacobian in every coupling iteration with data obtained from the coupling iterations and from previous time steps. The algorithm for the update of the Jacobian is constructed as follows:

At the beginning of the simulation, all the variables are equal to zero (in particular $\Delta \ddot{\boldsymbol{\theta}}^{a}, \boldsymbol{\Delta} \ddot{\boldsymbol{\theta}}^{b}, \boldsymbol{\Delta} \boldsymbol{M}^{a}, \boldsymbol{\Delta} \boldsymbol{M}^{b}$ and all the components of the Jacobian). Note that with such a zero Jacobian a Gauss-Seidel iteration without relaxation is retrieved (as can be seen in Equation (18) with $\boldsymbol{J}^{n+1, k}=\mathbf{0}$ ). Also, when going to each new time step, the data vectors $\left(\boldsymbol{\Delta} \ddot{\boldsymbol{\theta}}^{a}, \boldsymbol{\Delta} \ddot{\boldsymbol{\theta}}^{b}, \boldsymbol{\Delta} \boldsymbol{M}^{a}\right.$ and $\boldsymbol{\Delta} \boldsymbol{M}^{\boldsymbol{b}}$ ) are given the zero-value (cfr. Section 2.3.6).

The data obtained in the first coupling iteration $(k=0)$ of each time step are used as the reference (i.e. $\ddot{\boldsymbol{\theta}}^{\text {ref }}$ and $\boldsymbol{M}^{\text {ref }}$ ) for the Jacobian update. Also, in this first coupling iteration a first estimation of the Jacobian is obtained from an extrapolation of previous time steps (cfr. Section 2.3.5.2).

In each of the following coupling iterations $(k>0)$, this Jacobian can be updated with useable data obtained in the coupling iterations. First, it is checked whether or not the data of the current coupling iteration $k$ are useable as an acceleration perturbation vector. Subsequently, the Jacobian can be updated, which is done in three ways, 
depending on the acceleration perturbation vectors $\Delta \ddot{\boldsymbol{\theta}}^{a}$ and $\Delta \ddot{\boldsymbol{\theta}}^{b}$ available from the (current and previous) coupling iterations (cfr. Appendix 2):

- If no appropriate acceleration perturbation vectors are available, then the Jacobian cannot be updated and thus the extrapolated Jacobian is still used;

- Furthermore, if one acceleration perturbation vector is available, then the Jacobian can be updated. The data for the other acceleration perturbation vector are obtained from the acceleration perturbation vectors of previous time steps, or this data can be calculated by the extrapolated Jacobian;

- Finally, if both acceleration perturbation vectors are available, the Jacobian is calculated with both data and becomes independent of data from previous time steps.

A detailed description of the Jacobian update can be found in Appendix 2.

In the following, the other components of the new quasi-Newton algorithm will be discussed. The flow chart of this algorithm is visualized in Figure 2. It is noted that this flow chart is an extension of the basic flow chart in Figure 1.

\subsubsection{Check FSI convergence}

The subsequent coupling iterations are performed until the dynamic equilibrium at the fluid-structure interface is achieved. This equilibrium is checked by the FSI convergence criterion. For each leaflet $i$, this FSI convergence criterion is given by:

$$
\left|M_{i}^{n+1, k}-I_{i} \cdot \ddot{\theta}_{i}^{n+1, k}\right|<\varepsilon_{0}
$$

The FSI convergence threshold $\varepsilon_{0}$ is set relative to a reference moment $M_{\text {ref }}$, for example,

$$
\varepsilon_{0}=\frac{M_{r e f}}{1 \mathrm{E} 4}
$$

This reference moment $M_{\text {ref }}$ sets the minimal accuracy of the dynamic equilibrium needed to capture the general leaflet dynamics.

When the FSI convergence criterion (Equation (29)) is fulfilled, a new time step is initiated. However, when the FSI convergence criterion is not fulfilled, then a new coupling iteration $(k+1)$ is started (cfr. Section 2.3.4).

\subsubsection{Calculate angular accelerations of the new coupling iteration}

Before calculating the appropriate angular accelerations $\ddot{\theta}_{i}^{n+1, k+1}$ for the new coupling iteration $k+1$, the quality of the Jacobian is checked by evaluating the residual drop of the FSI convergence criterion of the last two coupling iterations. For example, an arbitrary criterion of two orders of magnitude in residual drop between these two subsequent coupling iterations is used:

$$
\left|M_{i}^{n+1, k}-I_{i} \cdot \ddot{\theta}_{i}^{n+1, k}\right|<\frac{\left|M_{i}^{n+1, k-1}-I_{i} \cdot \ddot{\theta}_{i}^{n+1, k-1}\right|}{100}
$$

When this expression is fulfilled, then the quality of the Jacobian of coupling iteration $k-1$ is good. Therefore, it can be concluded that the Jacobian of the current coupling iteration $k$ will probably also result in fast convergence, since this Jacobian can only become an improvement of the Jacobian of coupling iteration $k-1$. This is because when the Jacobian is updated in coupling iteration $k$, this update is done with more (or equally) relevant data (i.e. data of time step $n+1$ and not of time step $n$ ) than was available in coupling iteration $k-1$. Subsequently, the angular accelerations of the 
next coupling iteration $k+1$ are calculated by Equation (17) with the Jacobian of coupling iteration $k$ and this next coupling iteration is initiated.

However, when Equation (31) is not fulfilled, then the quality of the Jacobian of coupling iteration $k-1$ is insufficient. Therefore, it is uncertain whether or not the Jacobian of coupling iteration $k$ will result in fast convergence. The angular accelerations of the next coupling iteration $\ddot{\theta}_{i}^{n+1, k+1}$ are thus chosen in such a way that it will certainly become possible to calculate a good Jacobian in the next coupling iteration by the acceleration and moment data generated in this next coupling iteration.

The angular acceleration of this extra coupling iteration is thus chosen in one of three possible ways, depending on the acceleration perturbation vectors $\Delta \ddot{\boldsymbol{\theta}}^{a}$ and $\boldsymbol{\Delta} \ddot{\boldsymbol{\theta}}^{b}$ already obtained in the coupling iterations (cfr. Appendix 3):

- If no acceleration perturbation vectors are available from the coupling iterations (i.e $\boldsymbol{\Delta} \ddot{\boldsymbol{\theta}}^{a}=\mathbf{0}$ and $\boldsymbol{\Delta} \ddot{\boldsymbol{\theta}}^{b}=\mathbf{0}$ ), then the angular acceleration of one leaflet is perturbed with a significant parameter $\delta$ in the next coupling iteration (as is described in section 2.3.1);

- Furthermore, if one acceleration perturbation vector is already available (i.e. $\Delta \ddot{\boldsymbol{\theta}}^{a} \neq \mathbf{0}$ and $\boldsymbol{\Delta} \ddot{\boldsymbol{\theta}}^{b}=\mathbf{0}$ ), then the leaflet accelerations of the next coupling iteration are perturbed in a direction that is perpendicular to $\Delta \ddot{\boldsymbol{\theta}}^{a}$;

- Finally, if both acceleration perturbation vectors are available (i.e $\boldsymbol{\Delta} \ddot{\boldsymbol{\theta}}^{a} \neq \mathbf{0}$ and $\left.\Delta \ddot{\boldsymbol{\theta}}^{b} \neq \mathbf{0}\right)$, then no extra coupling iteration is needed. Therefore, the Jacobian is kept since the slow residual drop rate is inherent to the problem and will mostly not be efficiently resolved by generating extra data. The angular acceleration of the next coupling iterations are thus calculated by Equation (17).

A detailed description of these three cases can be found in Appendix 3.

\subsubsection{Increasing the efficiency}

The efficiency of the algorithm is increased in several ways. In the following, the use of a variable time step size and the extrapolation of data from previous time steps are discussed.

2.3.5.1 Variable time step size. Since the heart valve is most of the time in the closed or open position, its leaflets are only moving in a very small fraction of the time cycle. However, when the leaflets are moving, very small time steps are needed for reasons of accuracy. Therefore, there is a clear advantage in the use of a variable time step size which allows a relatively large time step size when the valve is at rest, and a smaller time step size when the valve is moving, since it decreases the total number of time steps in a time cycle and thus lowers the computational cost. For these reasons, a variable time step size is commonly used when simulating heart valves (Bang et al. 2006; Choi et al. 2009; De Tullio et al. 2009; Hong et al. 2009).

When the leaflets are moving, the maximum allowable time step size is restricted by the maximum allowable mesh motion, and thus by grid characteristics. This is due to limitations of the mesh motion algorithm, since the remeshing occurs when going to a new time step, and in the coupling iterations within a time step only grid node relocation can be used. So, there is a maximum allowable mesh motion in order to retain an accurate and quality mesh (and, in worst case, to avoid highly skewed or even inverted cells). 
A criterion for the time step size can be proposed based on this maximum leaflet movement $\left(\Delta \theta_{\max }\right)$ :

$$
\dot{\theta}_{i}^{n} \cdot \Delta t^{n+1} \leq \Delta \theta_{\max } \quad \Rightarrow \quad \Delta t^{n+1} \leq \min _{i=1,2}\left(\frac{\Delta \theta_{\max }}{\dot{\theta}_{i}^{n}}\right)
$$

When the leaflet velocity is large, then the time step size will be small and vice versa. Furthermore, the time step size is limited by a maximal value $\Delta t^{\max }$, otherwise the time step size would become infinite when none of the leaflets is moving (i.e. zero velocity).

2.3.5.2 Quadratic extrapolation from previous time steps. When advancing to a new time step, the angular accelerations for the first coupling iteration $(k=0)$ are estimated based on a quadratic extrapolation from previous time steps. This initial prediction of the values results in fewer coupling iterations per time step.

Also, the Jacobian's components are quadratically extrapolated from previous time steps. This is meaningful since the Jacobian is not changing much per time step.

Therefore, the extrapolation of data from previous time steps decreases the number of coupling iterations per time step and lowers the computational cost.

\subsubsection{Initialisation at the beginning of a new time step}

When going to a new time step $n+1$, the variables are initialized. This is done by setting $t^{n+1}=t^{n}+\Delta t^{n+1}, \Delta \ddot{\boldsymbol{\theta}}^{a}=\mathbf{0}, \Delta \ddot{\boldsymbol{\theta}}^{b}=\mathbf{0}, \Delta \boldsymbol{M}^{a}=\mathbf{0}, \Delta \boldsymbol{M}^{\boldsymbol{b}}=\mathbf{0}$ and $k=0$.

\subsubsection{Time integration scheme and leaflet behaviour at the limited position}

2.3.7.1 Backward Euler time integration. The angular velocity and angular position of each leaflet on the time level $n+1$ are calculated from the angular accelerations using a time-integration scheme. Because the flow solver in which the algorithm is implemented uses a backward Euler scheme, this scheme is also preferred for the structural movement:

$$
\left\{\begin{array} { l } 
{ \ddot { \theta } _ { i } ^ { n + 1 , k + 1 } = \frac { \dot { \theta } _ { i } ^ { n + 1 , k + 1 } - \dot { \theta } _ { i } ^ { n } } { \Delta t } } \\
{ \dot { \theta } _ { i } ^ { n + 1 , k + 1 } = \frac { \theta _ { i } ^ { n + 1 , k + 1 } - \theta _ { i } ^ { n } } { \Delta t } }
\end{array} \Rightarrow \left\{\begin{array}{l}
\dot{\theta}_{i}^{n+1, k+1}=\dot{\theta}_{i}^{n}+\Delta t \cdot \ddot{\theta}_{i}^{n+1, k+1} \\
\theta_{i}^{n+1, k+1}=\theta_{i}^{n}+\Delta t \cdot \dot{\theta}_{i}^{n}+\Delta t^{2} \cdot \ddot{\theta}_{i}^{n+1, k+1}
\end{array}\right.\right.
$$

If the time discretization schemes of the flow and the structural solver do not match, then spurious oscillations can occur, as is shown in Vierendeels et al. (2005).

2.3.7.2 Leaflet behaviour in open and closed position. The angular position of each leaflet is restricted by a minimum and a maximum angle, indicating, respectively, the fully closed and fully open position. In reality, this is usually achieved by a physical blocking mechanism incorporated in the design of the leaflet hinges.

These limitations can be numerically modelled by setting the angular position equal to the limited position and the angular velocity and angular acceleration equal to zero, as briefly suggested by Guivier et al. (2007). However, they did not explain how to make the transition from a moving to a resting leaflet.

In our algorithm, this transition is modelled by setting the position equal to the limitation and recalculating the angular velocity and acceleration. This is implemented as follows. When the calculated position of a leaflet exceeds its limitations (i.e. $\theta_{i}^{n+1, k+1}<\theta_{\min }$ or $\theta_{i}^{n+1, k+1}>\theta_{\max }$ ), then the position is set equal to this limitation (i.e. $\theta_{i}^{n+1, k+1}=\theta_{\min }$ or $\theta_{i}^{n+1, k+1}=\theta_{\max }$ ). In order to move exactly to the limited position in the 


\section{Results}

\subsection{Geometry setup}

The new quasi-Newton algorithm is used to simulate two 3D geometries containing the same BMHV. This BMHV is a model of the $25 \mathrm{~mm}$ ATS Open Pivot ${ }^{\mathrm{TM}}$ Standard Heart Valve in aortic position with the orifice inner diameter measuring $20.8 \mathrm{~mm}$. The valve is simplified at the hinge regions by cutting away the blocking mechanism and hinges at the casing. The valve is tested in two geometries. The first geometry consists of a rigid straight tube and is visualized in Figure 3. The second geometry also consists of a rigid straight tube upstream of the valve, but rigid Valsalva sinuses are added downstream of the valve (Figure 4). Such Valsalva sinuses are anatomically present in the ascending aortic root and have an influence on the valve closing. The Valsalva sinuses are based on the geometry described in Reul et al. (1990) and are positioned asymmetrically with respect to the leaflets rotation axes, in such a way that one of the leaflets faces directly one sinus. It is expected that this asymmetrical geometry induces an asymmetric flow field downstream of the valve, resulting in an asynchronous motion of the valve leaflets. Therefore, it is an effective test for the algorithm with two degrees of freedom. 
For both geometries, the upstream tube has a diameter of $22 \mathrm{~mm}$ and measures $75 \mathrm{~mm}$ in length. The downstream geometry has a length of $95 \mathrm{~mm}$. The diameter of the downstream tube is $27.36 \mathrm{~mm}$ for the Valsalva sinuses and $22 \mathrm{~mm}$ for the straight tube.

It is noted that both geometries are based on medical practice since, when implanting the BMHV, the surgeon can choose to preserve the Valsalva sinuses or to cut them away and replace the entire ascending aortic root (the so-called Bentall procedure (Bentall et al. 1968)).

\subsection{Case setup}

The quasi-Newton algorithm is implemented into the black box flow solver FLUENT $^{\mathrm{TM}}$ (v12.0.1) by the use of a script ("journal file") and user-written routines ("user-defined functions").

An unstructured grid is generated in both geometries and the ALE-approach is followed. The grid consists of approximately 800000 tetrahedral cells for both geometries. Two cell layers are generated in the gap (measuring $0.1 \mathrm{~mm}$ ) between the leaflets and the casing near the hinge region. The update of the grid is done in FLUENT $^{\mathrm{TM}}$ using its local remeshing method and spring-based smoothing. Due to the ALE approach, this remeshing can only be performed when going to a new time step. During the subsequent coupling iterations, the grid motion is performed with springbased smoothing in order to allow good mesh quality. The maximum time step size $\Delta t^{\max }$ is set to $0.001 \mathrm{~s}$.

An inlet aortic flow pulse with a period of 1s (displayed in Figure 5) is imposed upstream and was previously used in Dumont et al. $(2005,2007)$. The flow pulse profile is uniform. A physiological pressure profile is imposed at the downstream outlet boundary. Note, however, that in a rigid geometry the pressure level does not affect the flow field (only the pressure gradient appears in the equations).

Blood is modelled as a laminar incompressible Newtonian fluid with density and viscosity respectively equal to $1050 \mathrm{~kg} / \mathrm{m}^{3}$ and $4 \mathrm{E}-3 \mathrm{~Pa} \cdot \mathrm{s}$. A no-slip boundary condition is applied at the walls. The valve is initially set in the closed position. The moment of inertia of one rigid valve leaflet about its rotation axis is equal to $9.94 \mathrm{E}-9$ $\mathrm{kg} \cdot \mathrm{m}^{2}$.

Each simulation is run in parallel on eight cores ( 2 x Quad-Core Intel Xeon $2.66 \mathrm{GHz})$ with 8GB RAM.

\subsection{Simulation results}

The solution process shows that during valve movement the algorithm reaches convergence in each time step within approximately four coupling iterations (see Table 1, "opening" and "closing", with $\varepsilon_{0}=1 \mathrm{E}-13 \mathrm{Nm}$ ).

When analyzing the convergence process, it is noted that the data obtained in the first coupling iteration $(k=0)$ of each time step are the reference (i.e. $\ddot{\boldsymbol{\theta}}^{\text {ref }}$ and $\left.\boldsymbol{M}^{\text {ref }}\right)$ for the calculation of the acceleration perturbation vectors, as discussed in Section 2.3.2. It is found that the angular accelerations of the second coupling iteration $(k=1)$ are always used for the calculation of vector $\Delta \ddot{\boldsymbol{\theta}}^{a}$ for the Jacobian update. In $63 \%$ of the time steps during valve movement, the accelerations in the third coupling iteration $(k=2)$ are appropriate for the calculation of vector $\Delta \ddot{\boldsymbol{\theta}}^{b}$. Furthermore, in $12 \%$ of the time steps during valve movement, the Jacobian does not achieve a residual drop of two orders of magnitudes between two subsequent coupling iterations, and an extra coupling iteration is initiated, in order to achieve good quality of the Jacobian. The initiation of 
an extra coupling iteration with a perturbation of the accelerations equal to the small parameter $\delta$ only occurs when one leaflet is at rest in a limited position, while the other leaflet is still moving. In all other cases that the residual drop of Equation (31) is not fulfilled, the angular accelerations of the next coupling iteration are chosen perpendicular to vector $\Delta \ddot{\boldsymbol{\theta}}^{a}$. This data then results in a Jacobian with a better residual drop rate.

The results of the simulations are depicted in Figure 5 and Figure 6. The angular positions of the leaflets are presented in Figure 5, relative to the fully open position. Therefore, $0 \%$ and $100 \%$ refer, respectively, to the fully closed and fully open position. However, the results show that this maximum opening position is not reached in the second geometry with Valsalva sinuses. Such incomplete opening for the ATS Open Pivot ${ }^{\mathrm{TM}}$ Standard Heart Valve in a divergent geometry was also observed in Feng et al. (1999, 2000, 2001), Aoyagi et al. (2006) and, more recently, Sezai et al. (2009). Furthermore, for the Valsalva sinuses geometry, it is found that the two leaflets show differences in movement. Both results will be discussed in Section 4.1.

Figure 6 shows the velocity magnitudes at different time levels for the two geometries. Downstream of the valve, the flow pattern consists of three jets, which is typical for a BMHV.

\subsection{Sensitivity analysis}

In this section, the sensitivity with respect to the used criterion thresholds, the time step size and the grid size are discussed.

\subsubsection{Influence of the criterion thresholds $\varepsilon_{0}, \varepsilon_{1}$ and $\varepsilon_{2}$}

The algorithm is characterized by three thresholds, namely the FSI convergence threshold $\varepsilon_{0}$, the flow solver convergence threshold $\varepsilon_{1}$ and the Jacobian update threshold $\varepsilon_{2}$.

The FSI convergence threshold $\varepsilon_{0}$ is correlated to the reference value $M_{\text {ref, }}$, as described in section 2.3.3. For example, the equilibrium of Newton's Second Law is solved accurate to:

$$
\varepsilon_{0}=\frac{M_{r e f}}{1 \mathrm{E} 4}=\frac{1 \mathrm{E}-9}{1 \mathrm{E} 4}=1 \mathrm{E}-13 \mathrm{Nm}
$$

The flow solver convergence threshold $\varepsilon_{1}$ is the accuracy at which the moments are calculated in the flow solver. It is set relative to the FSI convergence threshold. For example, it is two orders of magnitudes smaller than the FSI convergence threshold:

$$
\varepsilon_{1}=\frac{\varepsilon_{0}}{100}=1 \mathrm{E}-15 \mathrm{Nm}
$$

The Jacobian update threshold $\varepsilon_{2}$ checks the direction and magnitude of the two acceleration perturbation vectors for the Jacobian update (see Section 2.3.2).

A sensitivity analysis will now be performed to obtain the optimal values for each of the thresholds $\varepsilon_{1}$ and $\varepsilon_{2}$.

The value of the flow solver convergence threshold $\varepsilon_{1}$ is called "optimal" if this value results in the smallest number of coupling iterations per time step within a reasonable amount of time.

The Jacobian update threshold $\varepsilon_{2}$ is called "optimal" if its value results in the smallest number of coupling iterations per time step (i.e. it is the most efficient) under the 
restriction that the calculated Jacobian needs to be of sufficient quality for all the time steps (i.e. all time steps need to converge).

In the following, the opening of the valve in the Valsalva sinuses geometry $(t=0 \mathrm{~s} . .0 .125 \mathrm{~s})$ is simulated in 177 time steps with variable time step size. The sensitivity analysis is done in two steps.

(1) In the first step, the FSI convergence threshold $\varepsilon_{0}$ is kept fixed at $1 \mathrm{E}-13 \mathrm{Nm}$ and the flow solver convergence threshold $\varepsilon_{1}$ and the Jacobian update threshold $\varepsilon_{2}$ are optimized. Therefore, simulations are performed in which $\varepsilon_{1}$ and $\varepsilon_{2}$ are varied in order to find their optimal values.

In the first set of simulations, $\varepsilon_{1}$ is kept fixed at $\varepsilon_{0} / 100$, and $\varepsilon_{2}$ is varied. An optimal value for $\varepsilon_{2}$ is found equal to 0.001 , since it is the largest value at which every time step converges, as can be seen in Table 2 .

In the second set of simulations, the value of $\varepsilon_{1}$ is varied, while keeping $\varepsilon_{2}$ fixed at 0.001 . The results are shown in Table 3. The value of $\varepsilon_{1}=\varepsilon_{0} / 100$ is obtained as "optimal", since it needs the smallest number of coupling iterations (within a reasonable amount of time).

So, it can be concluded that the optimal values are:

$$
\begin{aligned}
\varepsilon_{1} & =\frac{\varepsilon_{0}}{100} \\
\varepsilon_{2} & =0.001
\end{aligned}
$$

(2) Secondly, the FSI convergence threshold $\varepsilon_{0}$ is varied and it is investigated whether or not the obtained values for the thresholds $\varepsilon_{1}$ and $\varepsilon_{2}$ remain optimal. The FSI convergence threshold is set equal to $1 \mathrm{E}-10 \mathrm{Nm}$ :

$$
\varepsilon_{0}=\frac{M_{r e f}}{10}=\frac{1 \mathrm{E}-9}{10}=1 \mathrm{E}-10 \mathrm{Nm}
$$

which results in Table 4.

It is seen that the optimal values remain optimal and are not sensitive to the value of $\varepsilon_{0}$. Therefore, the values of $\varepsilon_{0} / \varepsilon_{1}$ and $\varepsilon_{2}$ are kept fixed at these optimal values and $\varepsilon_{0}$ is allowed to be varied by the user, according to the desired accuracy.

\subsubsection{Influence of the temporal discretization}

The influence of the time step size is analysed by varying the time step size criterion. Simulations with three different time step sizes are conducted for the straight tube geometry. The first simulation is done with the reference variable time step size, given by Equation (32).

In the second simulation, the variable time step size is halved. Therefore, the criterion becomes:

$$
\dot{\theta}_{i}^{n} \cdot \Delta t^{n+1} \leq \frac{\Delta \theta_{\max }}{2} \quad \Rightarrow \quad \Delta t^{n+1} \leq \min _{i=1,2}\left(\frac{\Delta \theta_{\max }}{2 \cdot \dot{\theta}_{i}^{n}}\right)
$$

Finally, a simulation is performed with a constant time step size:

$$
\Delta t^{n+1}=C s t=0.0005 \mathrm{~s}
$$

However, it is noted that when using this rather large constant time step size, a complete time cycle cannot be calculated. This is because during the closing of the valve, the leaflet velocity becomes too large so that the maximum leaflet displacement 
$\Delta \theta_{\max }$ is exceeded and the mesh motion fails. Therefore, this simulation is aborted at $t=0.38 \mathrm{~s}$.

The values for the angular accelerations and angular positions are compared at different time levels during the opening and closing of the valve in the straight tube geometry. The data are taken at $t=0.01 \mathrm{~s}, 0.02 \mathrm{~s}, 0.03 \mathrm{~s}, 0.04 \mathrm{~s}, 0.05 \mathrm{~s}, 0.06 \mathrm{~s}$ and $0.07 \mathrm{~s}$ for the opening phase and at $t=0.33 \mathrm{~s}, 0.34 \mathrm{~s}, 0.35 \mathrm{~s}, 0.36 \mathrm{~s}, 0.37 \mathrm{~s}$ and $0.38 \mathrm{~s}$ for the closing phase.

Subsequently, the averaged deviations (and their variances) between the three kinds of time step sizes are calculated in terms of percentage, relative to the variable time step size of Equation (32). This leads to the following results.

When comparing the variable time step size of Equation (32) with the variable time step size of Equation (43), an averaged deviation of $-0.6936 \%$ is calculated for the angular accelerations, with a variance of $1.6109 \%$. For the angular positions, the averaged deviation is $0.6280 \%$ with a variance of $0.0093 \%$

The variable time step size of Equation (32) is evaluated with the constant time step size of Equation (44). An averaged deviation of $-3.0020 \%$ is calculated for the angular accelerations, with a variance of $1.4318 \%$. The angular positions result in an averaged deviation of $0.2332 \%$ with a variance of $0.0045 \%$.

Therefore, it is concluded that time step size convergence is achieved for the straight tube geometry.

\subsubsection{Influence of the spatial discretization}

Subsequently, a spatial (grid) convergence study is performed. This is done for the straight tube geometry by constructing two grids with different sizes. The grids are constructed by defining the mesh size at the leaflet walls. Subsequently, a size function is applied at these leaflet walls, which means that in the direction perpendicular to the leaflet walls, the grid size in the geometry increases at a specified rate.

The first grid is the grid that is used in previous simulations, so the gap between leaflets and casing is meshed with two cell rows. Therefore, the grid measures 0.05 $\mathrm{mm}$ at the leaflet walls. It consists in total of approximately 800000 tetrahedral cells and is denoted as the "fine grid".

In the second grid, one cell layer is constructed in the gap between leaflets and casing. The grid has thus size $0.1 \mathrm{~mm}$ at the leaflets and is composed of approximately 400 000 tetrahedral cells. In the following, this grid is referred to as the "coarse grid".

The values for the angular accelerations and angular positions are compared at different time levels during the opening and closing of the valve. The data are taken at $t=0.01 \mathrm{~s}, 0.02 \mathrm{~s}, 0.03 \mathrm{~s}, 0.04 \mathrm{~s}, 0.05 \mathrm{~s}, 0.06 \mathrm{~s}$ and $0.07 \mathrm{~s}$ for the opening phase and at $t=0.33 \mathrm{~s}, 0.34 \mathrm{~s}, 0.35 \mathrm{~s}, 0.36 \mathrm{~s}, 0.37 \mathrm{~s}$ and $0.38 \mathrm{~s}$ for the closing phase. The averaged deviation (and variance) between both grids is calculated in terms of percentage, relative to the coarse grid. For the angular accelerations, an averaged deviation of $-0.6957 \%$ is calculated, with a variance of $1.3437 \%$. For the angular positions, the averaged deviation is $0.6215 \%$, with a variance of $0.0017 \%$.

It is concluded that grid convergence is achieved for the straight tube geometry.

\subsection{Comparison with existing coupling schemes}

In this section, the quasi-Newton algorithm and the existing coupling schemes (described in Section 2.2) are compared through numerical experiments. These results 
will be discussed in section 4.2, evaluating the stability, efficiency and characteristics of the coupling schemes.

\subsubsection{Strong coupling}

First, the stability of the solution process with strong coupling is studied. When no relaxation is used (i.e. $\omega_{i}{ }^{n+1, k}=1$ ), the simulation quickly diverges within a few coupling iterations of the time step. This demonstrates that relaxation is necessary.

In the following, the relaxation methods are evaluated. First, fixed relaxation is compared with Aitken relaxation. Subsequently, the comparison between Aitken relaxation and the quasi-Newton method is discussed.

3.5.1.1 Fixed relaxation versus dynamic Aitken relaxation. For the fixed relaxation factor, simulations are conducted for three leaflet positions of the valve in the straight tube geometry: $1 \%$ open leaflets, $5 \%$ open and, finally, the $99 \%$ open valve. For each of these leaflet positions, the value of the relaxation factor is varied and one time step is simulated. The associated needed numbers of coupling iterations are depicted in Figure 7. Thus, the allowable range of the fixed relaxation factor value for each leaflet position becomes visible.

The results clearly show that the optimal relaxation value increases with increasing leaflet position. For the $1 \%$ open valve, the optimal relaxation value is observed around 0.15 and this optimum increases to 0.36 for the $99 \%$ open valve. Also, the maximum allowable relaxation factor, in order to obtain convergence, increases as function of the leaflet position, ranging from 0.235 for the $1 \%$ open valve to 0.675 for the $99 \%$ open leaflets.

When using Aitken relaxation, a relaxation factor of 0.149 was also found in the first time step. In the following time steps, the Aitken relaxation factor rises steeply when the valve opens, until the stable value of approximately 0.375 is obtained, achieving convergence within 3 to 4 coupling iterations for each time step, as depicted in Figure 8.

It is noted that the value of the stable Aitken relaxation factor, i.e. approximately 0.375 , is of the same magnitude as the Aitken relaxation factors computed in Borazjani et al. (2008). This study also reported that the Aitken relaxation factors calculated during the closing phase are generally larger than those during the opening phase, due to a stabilizing effect caused by the flow. However, this is not seen in our results, since our computed Aitken relaxation factors are not very sensitive to the movement phase, as visualized in Figure 8.

3.5.1.2 Aitken relaxation versus quasi-Newton method. In this paragraph, the dynamic relaxation techniques are compared with each other. The needed number of coupling iterations (with $\varepsilon_{0}=1 \mathrm{E}-13 \mathrm{Nm}$ ) can be found in Table 1 for the Valsalva sinuses geometry and for the straight tube geometry. In order to make a fair comparison of the efficiency, the Aitken relaxation factor for the first coupling iteration $(k=0)$ is estimated from a quadratic extrapolation from previous time steps. Aitken relaxation needs on average 3.2 coupling iterations per time step for the straight tube geometry and 3.8 coupling iterations per time step for the Valsalva sinuses geometry. However, when using the new quasi-Newton method, convergence is achieved in 2.3 coupling iterations per time step for the straight tube and 2.7 coupling iterations for the geometry with the Valsalva sinuses. Therefore, the Aitken relaxation method needs on average $40 \%$ more coupling iterations than the quasi-Newton method. 
Subsequently, the sensitivity to the FSI convergence threshold is compared in Table 1. For the Aitken relaxation, a positive relation is found between the needed number of coupling iterations and the order of magnitude of the FSI convergence threshold. When the FSI convergence threshold increases by an order of magnitude, this results on average in 0.25 fewer coupling iterations.

For the quasi-Newton method, a positive relation is also found, resulting in an increase of the needed number of coupling iterations with increasing order of magnitude of the FSI convergence threshold. However, when the FSI convergence threshold increases by an order of magnitude, on average 0.075 fewer coupling iterations are needed. Thus, its slope is less steep than is the case with Aitken relaxation. This is because when the FSI convergence threshold decreases by one order of magnitude, this leads to an increase of the number of times steps in which the residual drop of two orders of magnitude is not achieved and thus to an increase of the needed extra coupling iterations with (perpendicular) acceleration perturbations. With $\varepsilon_{0}=1 \mathrm{E}-13 \mathrm{Nm}$, for example, such an extra coupling iteration was initiated in $12 \%$ of the time steps (as described in Section 3.3). With $\varepsilon_{0}=1 \mathrm{E}-12 \mathrm{Nm}$, this percentage rised to $33 \%$, leading to $46 \%$ for $\varepsilon_{0}=1 \mathrm{E}-11 \mathrm{Nm}$. This increase can be visualized in the plots of the convergence process for $\varepsilon_{0}=1 \mathrm{E}-11 \mathrm{Nm}$ (Figure 9) and $\varepsilon_{0}=1 \mathrm{E}-13 \mathrm{Nm}$ (Figure 10). Figure 9 shows that a perpendicular acceleration perturbation vector was initiated in the third coupling iteration in three time steps (i.e. time step 5, 9 and 10) and in the fourth coupling iteration in one time step (i.e. time step 7).

\subsubsection{Loose coupling}

The analysis of the loose coupling method is started by examining the stability of the solution process. When no relaxation is used (i.e. $\omega_{i}^{n+1}=1$ ), the simulation diverges within a few time steps. Therefore, the necessity of relaxation is acknowledged. The relaxation factor is subsequently lowered, until convergence is found. A factor value lower than or equal to 0.36 is seen to be stable. This threshold value is consistent with Borazjani et al. (2008), but is in contrast to the values used in (Redaelli et al. 2004; Nobili et al. 2007) in which instability was found in the first time steps as soon as the relaxation factor exceeded 0.07 . This discrepancy signals the case-specificity of the relaxation factor and, therefore, expresses the need for a dynamic factor.

Simulations of the opening phase for the straight tube geometry have been conducted with a constant time step size $(\Delta t=0.0005 \mathrm{~s})$ and $\omega_{i}{ }^{n+1}$, subsequently, equal to $0.1,0.25$ and 0.325 . The grid- and time step converged solution calculated with the strong coupling methods is accepted as the reference solution for the valve movement in the straight tube geometry. These strong coupling leaflet movements are compared with the solutions obtained by loose coupling, as visualized in Figure 11. The results show that the leaflet movement, obtained by the loose coupling, is characterised by spurious oscillations and is lagged in time. Subsequently, the pressure field, the velocity field and the velocity vectors at the b-datum gap (i.e. the gap between the closed leaflets) are depicted for subsequent time steps in Figure 12 for the loose coupling (with $\omega_{i}^{n+1}=0.325$ ) and in Figure 13 for the strong coupling (quasi-Newton method). It shows that, in contrast to strong coupling, the flow fields obtained by the loose coupling also contain oscillations.

These results will be discussed in Section 4.2.2. 


\section{Discussion}

In this section, the leaflet kinematics observed in the numerical simulations of Section 3.3 are discussed. Subsequently, the comparison between the existing schemes in Section 3.5 is commented.

\subsection{Numerical simulation}

The results show that the presented algorithm is stable and efficient. Furthermore, Figure 5 shows that the maximum opening position of the valve leaflets is not reached in the second geometry with Valsalva sinuses. Such incomplete opening for the ATS Open Pivot ${ }^{\mathrm{TM}}$ Standard Heart Valve in a divergent geometry is explained due to the greater sensitivity of the leaflet movement to the flow field compared to other BMHV designs, since the leaflets extend farther in the flow downstream of the orifice than is the case in other designs (Feng et al. 1999). Therefore, the valve does not open completely in the divergent transvalvular flow caused by the Valsalva sinuses enlargement, since the leaflets tend to align with the streamlines. In the straight tube, however, a full opening of the valve leaflets is observed.

This incomplete opening also explains the difference in the closing phase between the two geometries, since it is found that the valve in the Valsalva sinuses geometry is closed sooner. This is because the leaflets in the straight tube reach the completely open position and therefore need to rotate over a greater angle in order to close. Hence, they have a greater closing volume (Feng et al. 2000). Nevertheless, the moment in time at which the leaflets start to close is approximately the same for both geometries. These conclusions seem to be in contrast with Bang et al. (2006), who simulated the dynamics of a BMHV in a straight tube and compared it with a geometry containing symmetrically placed Valsalva sinuses. It was found that the valve with the Valsalva sinuses closed later than the straight tube, since there is more blood in the distal part of the heart valve. However, this seemingly contradiction can be explained by noting that opposed to our case, the valve was completely open in Bang et al. (2006) before it started to close (it was not the ATS Open Pivot ${ }^{\mathrm{TM}}$ Standard Heart Valve). The closing volume in the half-open position with Valsalva sinuses is thus always smaller than the closing volume of a valve in a completely open position and therefore the half-open valve always closes sooner, as is described in the experimental results performed by Feng et al. (2000) for different types of BMHVs.

Also, for the Valsalva sinuses geometry, it is observed that the two leaflets show differences in movement. As mentioned above, it can be understood that this asynchrony is triggered by the presence of the asymmetric geometry downstream of the valve. In the straight tube geometry, no differences in movement between the two leaflets are seen. The asynchronous leaflet movement caused by the asymmetrically placed Valsalva sinuses is consistent with De Tullio et al. (2009) and Hong et al. (2009).

\subsection{Evaluation of the existing coupling schemes}

\subsubsection{Strong coupling}

The results for the fixed relaxation factor show that the maximum allowable factor value increases as function of the opening angle (and hence the gap size between leaflet and casing). This is also the case for the optimal value of the relaxation factor. Such variation of the optimal relaxation value as a function of the leaflet position makes the selection of the fixed relaxation factor a trade-off between efficiency and 
stability. This can be explained as follows. The optimal value in the closed valve (i.e. $\left.\omega_{i}{ }^{n+1, k}=0.15\right)$ results in 16 needed coupling iterations. However, with this value, approximately 23 coupling iterations per time step are needed to achieve convergence as soon as the valve is a few degrees open, leading to 33 coupling iterations for the almost fully open case. The optimal value in the closed valve is thus far from optimal for the entire time cycle. Moreover, when setting the fixed relaxation factor equal to the optimal value of the open valve (i.e. $\omega_{i}^{n+1, k}=0.36$ ), this quickly leads to divergence in the closed position. Therefore, a fixed relaxation value is a trade-off value that needs to allow stability throughout the entire time cycle.

Furthermore, when using Aitken relaxation, the same optimal relaxation factors were achieved at each leaflet position, as depicted in Figure 8. Therefore, the results indicate that the dynamic (Aitken) relaxation technique outperforms the fixed relaxation in efficiency, since this dynamic factor is optimized for each specific time step (and coupling iteration) of the time cycle.

Subsequently, the quasi-Newton method is compared with the Aitken relaxation. In the results, it is found that the Aitken relaxation method needs on average $40 \%$ more coupling iterations than the quasi-Newton method. This increase in efficiency can be understood since in the quasi-Newton method the mutual influence of each leaflet is explicitly calculated. Therefore, the quasi-Newton method uses a relaxation matrix with four dynamic components, in contrast to only one dynamic Aitken relaxation factor. This makes the estimation of the angular accelerations between subsequent coupling iterations more accurate, thus resulting in faster convergence. It is concluded that the Aitken $\Delta^{2}$ relaxation method is more expensive than the quasi-Newton method.

\subsubsection{Loose coupling}

When comparing the leaflet movements obtained by strong coupling with the solutions obtained by loose coupling, as visualized in Figure 11, three comments can be made.

First, loose coupling is characterized by spurious oscillations in the leaflet movements, which are caused by a subsequent undershooting and overshooting in time, as also seen by Borazjani et al. (2008). These spurious oscillations originate from a sudden change at a time level and emerge due to the lack of feedback within a time step since this implies that the oscillations cannot be damped out within the subsequent coupling iterations of one time step but will be damped out in the subsequent time steps. A sudden change at a time level can have a physical cause, for example when a leaflet starts to move from a resting state (i.e. zero acceleration and velocity), or when the movement of the leaflet is suddenly blocked by the blocking mechanism.

Secondly, the results clearly show that the leaflet movement obtained with loose coupling is lagging in time, as also reported in Redaelli et al. (2004). This is due to the fact that the solution of the flow field at time step $n$ is used to calculate the movement of time step $n+1$.

Finally, the result depends on the chosen value of the relaxation parameter $\omega_{i}{ }^{n+1}$. It is observed that with increasing relaxation factor $\omega_{i}{ }^{n+1}$, the magnitude of the spurious oscillations increases and the time delay becomes smaller (Redaelli et al. 2004). So when the relaxation factor increases, the fit with the reference solution becomes better. However, when this factor becomes too large, then the solution diverges. This raises the question on how to define an optimal relaxation factor, and on how such an optimal value can be found a priori. Similar to the fixed strong 
coupling relaxation, it can thus be concluded that the choice of the loose coupling relaxation factor lacks a physical meaning.

Subsequently, an evaluation of the pressure and velocity magnitude contours and the velocity vectors (in the b-datum gap) is made in Figures 12 to 13.

For the loose coupling (with $\omega_{i}^{n+1}=0.325$ ), it is seen that the flow fields are changing rapidly in subsequent time steps. Due to the oscillations in the leaflet movement (as visualized in Figure 11), a significant fluid volume needs to flow from one side of the leaflet to the other side between subsequent time steps, leading to oscillations in the flow field and the pressure fields. So, the over- and undershooting of the leaflet movement feeds back to an unphysical over- and undershooting of the pressure gradient and the velocity magnitudes (in the b-datum gap). Some remarks can be made. First, in case of loose coupling, (unphysical) large velocity magnitudes can be found in the gaps, leading to unphysical large shear stresses. Therefore, when one wants to examine the blood damage, the loose coupling method is not recommended. Secondly, in Figure 11 it is observed that increasing the (loose coupling) relaxation factor leads to a better agreement with the physical solution. However, this is certainly not the case for the flow fields. With increasing loose coupling relaxation factor, the amplitude of the oscillations in the pressure gradients and the velocity magnitudes becomes larger. With large (unstable) relaxation factor, even backflow through the gaps could be observed.

\section{Conclusion}

A strong coupling algorithm for the partitioned simulation of a BMHV is presented. The convergence of the coupling iterations between the flow solver and the leaflet motion solver is accelerated using the Jacobian with the derivatives of the pressure and viscous moments acting on the leaflets with respect to the leaflet accelerations. This Jacobian is numerically calculated from the coupling iterations. An error analysis is performed to derive a criterion for the selection of such useable coupling iterations. Furthermore, the efficiency of the algorithm is increased by the extrapolation and reuse of data and the use of a variable time step size.

Subsequently, the algorithm is successfully tested and implemented for two 3D cases of a BMHV. The algorithm converges within a few coupling iterations. The results show that the leaflets in an asymmetric geometry move asynchronously. In such cases, an algorithm with two degrees of freedom is thus required.

Finally, a comparison is made with existing coupling schemes. It is observed that the developed coupling scheme outperforms these existing schemes in needed coupling iterations per time step and CPU time.

\section{Acknowledgements}

Sebastiaan Annerel is funded by a BOF-grant (Special Research Fund) from Ghent University Association. Joris Degroote gratefully acknowledges a postdoctoral fellowship of the Research Foundation - Flanders (FWO). 


\section{References}

Annerel S, Degroote J, Claessens T, Vierendeels J. 2010. Evaluation of a new Implicit Coupling Algorithm for the Partitioned Fluid-Structure Interaction Simulation of Bileaflet Mechanical Heart Valves. IOP Conf. Ser.: Mater. Sci. Eng. 10: 012124.

Aoyagi S, Arinaga K, Fukunaga S, Tayama E, Kosuga T, Akashi H. 2006. Leaflet Movement of the ATS Valve in the Aortic Position: Unique Behavior Observed in 19-mm Valves. Ann Thorac Surg. 82:853-857.

Astorino M, Gerbeau J-F, Pantz O, Traoré K-F. 2009. Fluid-structure interaction and multibody contact: Application to aortic valves. Comput Methods Appl Mech Engrg. 198:3603-3612.

Bang JS, Yoo SM, Kim CN. 2006. Characteristics of Pulsatile Blood Flow Through the Curved Bileaflet Mechanical Heart Valve Installed in Two Different Types of Blood Vessels: Velocity and Pressure of Blood Flow. ASAIO J. 52:234-242.

Bentall H, De Bono A. 1968. A technique for complete replacement of the ascending aorta. Thorax 23:338-339.

Borazjani I, Ge L, Sotiropoulos F. 2008. Curvilinear immersed boundary method for simulating fluid structure interaction with complex 3D rigid bodies. J Comput Phys. 227(16):7587-7620.

Causin P, Gerbeau J-F, Nobile F. 2005. Added-mass effect in the design of partitioned algorithms for fluid-structure problems. Comput Methods Appl Mech Engrg. 194:4506-4527.

Choi CR, Kim CN. 2009. Numerical Analysis on the Hemodynamics and Leaflet dynamics in a Bileaflet Mechanical Heart Valve Using a Fluid-structure Interaction Method. ASAIO J. 55(5):428-437.

Dahl SK, Vierendeels J, Degroote J, Annerel S, Hellevik LR, Skallerud B. 2010. FSIsimulation of asymmetric mitral valve dynamics during diastolic filling. Comput Method Biomec. DOI: 10.1080/10255842.2010.517200.

Degroote J, Bruggeman P, Haelterman R, Vierendeels J. 2008. Stability of a coupling technique for partitioned solvers in FSI applications. Comput Struct. 86:2224-2234.

Degroote J, Annerel S, Vierendeels J. 2010. Stability analysis of Gauss-Seidel iterations in a partitioned simulation of fluid-structure interaction. Comput Struct. 88:263-271.

De Tullio MD, Cristallo A, Balaras E, Verzicco R. 2009. Direct numerical simulation of the pulsatile flow through an aortic bileaflet mechanical heart valve. J Fluid Mech. 622:259-290.

Dos Santos ND, Gerbeau J-F, Bourgat J-F. 2008. A partitioned fluid-structure algorithm for elastic thin valves with contact. Comput Methods Appl Mech Engrg. 197:1750-1761.

Dumont K, Vierendeels J, Segers P, Van Nooten G, Verdonck P. 2005. Predicting ATS Open Pivot $^{\mathrm{TM}}$ Heart Valve Performance with Computational Fluid Dynamic. J Heart Valve Dis. 14:393-399.

Dumont K, Vierendeels J, Kaminsky R, Van Nooten G, Verdonck P, Bluestein D. 2007. Comparison of the Hemodynamic and Thrombogenic Performance of Two Bileaflet Mechanical Heart Valves Using a CFD/FSI Model. J Biomech Eng. 129:558-565.

Feng Z, Umezu M, Fujimoto T, Tsukahara T, Nurishi M, Kawaguchi D, Masuda S. 1999. Analysis of ATS leaflet behaviour by in vitro experiment. J Artif Organs. 2:46-52.

Feng Z, Umezu M, Fujimoto T, Tsukahara T, Nurishi M, Kawaguchi D. 2000. In Vitro Hydrodynamic Characteristics Among Three Bileaflet Valves in the Mitral Position. J Artif Organs. 24(5):346-352.

Feng Z, Nakamura T, Fujimoto T, Umezu M. 2001. Influence of valve size on the hydrodynamic performance of the ATS valve. J Artif Organs. 4:303-307.

Guivier C, Deplano V, Pibarot P. 2007. New insights into the assessment of the prosthetic valve performance in the presence of subaortic stenosis through a fluid-structure interaction model. J Biomech. 40:2283-2290. 
Guivier-Curien C, Deplano V, Bertrand E. 2009. Validation of a numerical 3-D fluid-structure interaction model for a prosthetic valve based on experimental PIV measurements. Med Eng Phys. 31:986-993.

Hong T-H, Choi C-R, Kim C-N. 2009. Characteristics of Hemodynamics in a Bileaflet Mechanical Heart Valve using an Implicit FSI Method. World Academy of Science, Engineering and Technology. 49:679-684.

Irons B M, Tuck RC. 1969. A version of the Aitken accelerator for computer iteration. Int J Numer Meth Eng. 1:275-277.

Küttler U, Wall W A. 2008. Fixed-point fluid-structure interaction solvers with dynamic relaxation. Comput Mech. 43:61-72.

Le Tallec P, Mouro J. 2001. Fluid structure interaction with large structural displacements. Comput Methods Appl Mech Engrg. 190:3039-3067.

Makhijani VB, Yang HQ, Dionne PJ, Thubrikar MJ. 1997. Three-Dimensional Coupled Fluid-Structure Simulation of Pericardial Bioprosthetic Aortic Valve Function. ASAIO J. 43(5):M387-M392.

Mok DP, Wall WA, Ramm E. 2001. Accelerated iterative substructuring schemes for instationary fluid-structure interaction. Computational Fluid and Solid Mechanics (K. Bathe, ed.), Elsevier, p. 1325-1328.

Morsi YS, Yang WW, Wong CS, Das S. 2007. Transient fluid-structure coupling for simulation of a trileaflet heart valve using weak coupling. J Artif Organs. 10:96-103.

Nobili M, Passoni G, Redaelli A. 2007. Two fluid-structure approaches for 3D simulation of St. Jude Medical bileaflet valve opening. J Appl Biomater Biom. 5:49-59.

Nobili M, Morbiducci U, Ponzini R, Del Gaudio C, Balducci A, Grigioni M, Maria Montevecchi F, Redaelli A. 2008. Numerical simulation of the dynamics of a bileaflet prosthetic heart valve using a fluid-structure interaction approaches. J Biomech. 41:2539-2550.

Penrose JMT, Staples CJ. 2002. Implicit fluid-structure coupling for simulation of cardiovascular problems. Int J Numer Meth Fl. 40:467-478.

Peskin C. 1972. Flow Patterns Around Heart Valves: A Numerical Method. J Comput Phys. 10:252-271.

Redaelli A, Bothorel H, Votta E, Soncini M, Morbiducci U, Del Gaudio C, Balducci A, Grigioni M. 2004. 3-D Simulation of the St. Jude Medical Bileaflet Valve Opening Process: Fluid-Structure Interaction Study and Experimental Validation. J Heart Valve Dis. 13:804-813.

Reul, H, Vahlbruch A, Giersiepen M, Schmitz-Rode Th, Hirtz V, Effert S. 1990. The geometry of the aortic root in health, at valve disease and after valve replacement. J Biomech. 23:181-191.

Sezai A, Umeda T, Hata M, Niino T, Unosawa S, Tokai K, Kasamaki Y, Minami K. 2009. A transesophageal Echocardiographic and Cine-Fluoroscopic Evaluation of an ATS Prosthetic Valve Opening. Surg Today. 39:300-305.

Sotiropoulos F, Borazjani I. 2009. A review of state-of-the-art numerical methods for simulating flow through mechanical heart valves. Med Biol Eng Comput. 47:245-56.

Tai CH, Liew KM, Zhao Y. 2007. Numerical simulation of 3D fluid-structure interaction flow using an immersed object method with overlapping grid. Comput Struct. 85:749-762.

Van Loon R, Anderson PD, de Hart J, Baaijens FPT. 2004. A combined fictitious domain/adaptive meshing method for fluid-structure interaction in heart valves. Int $\mathrm{J}$ Numer Meth Fl. 46:533-544.

Vierendeels J, Dumont K, Dick E, Verdonck P. 2005. Analysis and Stabilization of FluidStructure Interaction Algorithm for Rigid-Body Motion. AIAA J. 43:2549-2557.

Vierendeels J, Dumont K, Verdonck P. 2008. A partitioned strongly coupled fluid-structure interaction method to model heart valve dynamics. J Comput Appl Math. 215:602609.

Xia GH, Zhao Y, Yeo JH. 2009. Parallel unstructured multigrid simulation of 3D unsteady flows and fluid-structure interaction in mechanical heart valve using immersed membrane method. Comput Fluids 38(1):71-79. 


\section{Appendix 1: Error analysis}

The Jacobian update criterion $\varepsilon_{2}$ (Equation (28)) is derived from an error propagation analysis, as mentioned in Section 2.3.2. These errors are initiated as numerical errors in the flow solver. It is assumed that the input to the flow solver (i.e. the angular accelerations) is free of error and that all the numerical errors emerge at the output of the flow solver (i.e. the moments). Furthermore, these numerical errors are assumed to be independent of each other.

The moment can be written as the sum of its exact value (denoted by the bar) and its absolute error $(A E)$ :

$$
M=\bar{M} \pm A E(M)
$$

Since the convergence of the moments is checked, the worst case (i.e. with maximum error) can be proposed in which the numerical error is of the order of the flow solver convergence threshold $\varepsilon_{1}$ :

$$
\max _{i, j}\left(A E\left(M_{i}^{j}\right)\right)=\varepsilon_{1}
$$

The maximum absolute error of the differences in moments can then be calculated as being equal to $\sqrt{2} \cdot \varepsilon_{1}$ :

$$
\begin{aligned}
A E\left(\Delta M_{i}^{j}\right) \leq & \max _{i, j}\left(A E\left(\Delta M_{i}^{j}\right)\right) \\
& =\max _{i, j}\left(A E\left(M_{i}^{j}-M_{i}^{r e f}\right)\right) \\
& =\sqrt{\max _{i, j}\left(A E\left(M_{i}^{j}\right)\right)^{2}+\max _{i}\left(A E\left(M_{i}^{r e f}\right)\right)^{2}} \\
& =\sqrt{2} \cdot \varepsilon_{1}
\end{aligned}
$$

These errors are propagated to the calculated Jacobian components (Equation (23)) and the absolute errors of these components are calculated:

$$
\begin{aligned}
& A E\left(\frac{\Delta M_{1}}{\Delta \ddot{\theta}_{1}}\right)=\frac{\sqrt{\left(A E\left(\Delta M_{1}^{a}\right)\right)^{2} \cdot\left(\Delta \ddot{\theta}_{2}^{b}\right)^{2}+\left(A E\left(\Delta M_{1}^{b}\right)\right)^{2} \cdot\left(\Delta \ddot{\theta}_{2}^{a}\right)^{2}}}{\left|\Delta \ddot{\theta}_{2}^{b} \cdot \Delta \ddot{\theta}_{1}^{a}-\Delta \ddot{\theta}_{2}^{a} \cdot \Delta \ddot{\theta}_{1}^{b}\right|} \\
& A E\left(\frac{\Delta M_{1}}{\Delta \ddot{\theta}_{2}}\right)=\frac{\sqrt{\left(A E\left(\Delta M_{1}^{b}\right)\right)^{2} \cdot\left(\Delta \ddot{\theta}_{1}^{a}\right)^{2}+\left(A E\left(\Delta M_{1}^{a}\right)\right)^{2} \cdot\left(\Delta \ddot{\theta}_{1}^{b}\right)^{2}}}{\left|\Delta \ddot{\theta}_{2}^{b} \cdot \Delta \ddot{\theta}_{1}^{a}-\Delta \ddot{\theta}_{2}^{a} \cdot \Delta \ddot{\theta}_{1}^{b}\right|} \\
& A E\left(\frac{\Delta M_{2}}{\Delta \ddot{\theta}_{1}}\right)=\frac{\sqrt{\left(A E\left(\Delta M_{2}^{a}\right)\right)^{2} \cdot\left(\Delta \ddot{\theta}_{2}^{b}\right)^{2}+\left(A E\left(\Delta M_{2}^{b}\right)\right)^{2} \cdot\left(\Delta \ddot{\theta}_{2}^{a}\right)^{2}}}{\left|\Delta \ddot{\theta}_{2}^{b} \cdot \Delta \ddot{\theta}_{1}^{a}-\Delta \ddot{\theta}_{2}^{a} \cdot \Delta \ddot{\theta}_{1}^{b}\right|} \\
& A E\left(\frac{\Delta M_{2}}{\Delta \ddot{\theta}_{2}}\right)=\frac{\sqrt{\left(A E\left(\Delta M_{2}^{b}\right)\right)^{2} \cdot\left(\Delta \ddot{\theta}_{1}^{a}\right)^{2}+\left(A E\left(\Delta M_{2}^{a}\right)\right)^{2} \cdot\left(\Delta \ddot{\theta}_{1}^{b}\right)^{2}}}{\left|\Delta \ddot{\theta}_{2}^{b} \cdot \Delta \ddot{\theta}_{1}^{a}-\Delta \ddot{\theta}_{2}^{a} \cdot \Delta \ddot{\theta}_{1}^{b}\right|}
\end{aligned}
$$

which can be simplified as:

$$
\begin{aligned}
& A E\left(\frac{\Delta M_{1}}{\Delta \ddot{\theta}_{1}}\right) \leq \sqrt{2} \cdot \varepsilon_{1} \cdot \frac{\sqrt{\left(\Delta \ddot{\theta}_{2}^{b}\right)^{2}+\left(\Delta \ddot{\theta}_{2}^{a}\right)^{2}}}{\left|\Delta \ddot{\theta}_{2}^{b} \cdot \Delta \ddot{\theta}_{1}^{a}-\Delta \ddot{\theta}_{2}^{a} \cdot \Delta \ddot{\theta}_{1}^{b}\right|} \\
& A E\left(\frac{\Delta M_{1}}{\Delta \ddot{\theta}_{2}}\right) \leq \sqrt{2} \cdot \varepsilon_{1} \cdot \frac{\sqrt{\left(\Delta \ddot{\theta}_{1}^{a}\right)^{2}+\left(\Delta \ddot{\theta}_{1}^{b}\right)^{2}}}{\left|\Delta \ddot{\theta}_{2}^{b} \cdot \Delta \ddot{\theta}_{1}^{a}-\Delta \ddot{\theta}_{2}^{a} \cdot \Delta \ddot{\theta}_{1}^{b}\right|}
\end{aligned}
$$




$$
\begin{aligned}
& A E\left(\frac{\Delta M_{2}}{\Delta \ddot{\theta}_{1}}\right) \leq \sqrt{2} \cdot \varepsilon_{1} \cdot \frac{\sqrt{\left(\Delta \ddot{\theta}_{2}^{b}\right)^{2}+\left(\Delta \ddot{\theta}_{2}^{a}\right)^{2}}}{\left|\Delta \ddot{\theta}_{2}^{b} \cdot \Delta \ddot{\theta}_{1}^{a}-\Delta \ddot{\theta}_{2}^{a} \cdot \Delta \ddot{\theta}_{1}^{b}\right|} \\
& A E\left(\frac{\Delta M_{2}}{\Delta \ddot{\theta}_{2}}\right) \leq \sqrt{2} \cdot \varepsilon_{1} \cdot \frac{\sqrt{\left(\Delta \ddot{\theta}_{1}^{a}\right)^{2}+\left(\Delta \ddot{\theta}_{1}^{b}\right)^{2}}}{\left|\Delta \ddot{\theta}_{2}^{b} \cdot \Delta \ddot{\theta}_{1}^{a}-\Delta \ddot{\theta}_{2}^{a} \cdot \Delta \ddot{\theta}_{1}^{b}\right|}
\end{aligned}
$$

Equation (17) gives in full notation for the angular accelerations of each leaflet, with the finite difference approximation of the Jacobian:

$$
\left\{\begin{array}{c}
\ddot{\theta}_{1}^{n+1, k+1}=\frac{\frac{\Delta M_{1}}{\Delta \ddot{\theta}_{2}} M_{2}^{n+1, k}-\frac{\Delta M_{2}}{\Delta \ddot{\theta}_{2}} M_{1}^{n+1, k}-\frac{\Delta M_{1}}{\Delta \ddot{\theta}_{2}} \frac{\Delta M_{2}}{\Delta \ddot{\theta}_{1}} \ddot{\theta}_{1}^{n+1, k}-I_{2} \frac{\Delta M_{1}}{\Delta \ddot{\theta}_{2}} \ddot{\theta}_{2}^{n+1, k}+I_{2} M_{1}^{n+1, k}+\frac{\Delta M_{2}}{\Delta \ddot{\theta}_{2}} \frac{\Delta M_{1}}{\Delta \ddot{\theta}_{1}} \ddot{\theta}_{1}^{n+1, k}-I_{2} \frac{\Delta M_{1}}{\Delta \ddot{\theta}_{1}} \ddot{\theta}_{1}^{n+1, k}}{I_{1} I_{2}-I_{1} \frac{\Delta M_{2}}{\Delta \ddot{\theta}_{2}}-I_{2} \frac{\Delta M_{1}}{\Delta \ddot{\theta}_{1}}-\frac{\Delta M_{1}}{\Delta \ddot{\theta}_{2}} \frac{\Delta M_{2}}{\Delta \ddot{\theta}_{1}}+\frac{\Delta M_{2}}{\Delta \ddot{\theta}_{2}} \frac{\Delta M_{1}}{\Delta \ddot{\theta}_{1}}} \\
\ddot{\theta}_{2}^{n+, k+1}=\frac{-I_{1} \frac{\Delta M_{2}}{\Delta \ddot{\theta}_{1}} \ddot{\theta}_{1}^{n+1, k}+\frac{\Delta M_{2}}{\Delta \ddot{\theta}_{2}} \frac{\Delta M_{1}}{\Delta \ddot{\theta}_{1}} \ddot{\theta}_{2}^{n+1, k}-I_{1} \frac{\Delta M_{2}}{\Delta \ddot{\theta}_{2}} \ddot{\theta}_{2}^{n+1, k}-\frac{\Delta M_{1}}{\Delta \ddot{\theta}_{1}} M_{2}^{n+1, k}+I_{1} M_{2}^{n+1, k}+M_{1}^{n+1, k} \frac{\Delta M_{2}}{\Delta \ddot{\theta}_{1}}-\frac{\Delta M_{1}}{\Delta \ddot{\theta}_{2}} \frac{\Delta M_{2}}{\Delta \ddot{\theta}_{1}} \ddot{\theta}_{2}^{n+1, k}}{I_{1} I_{2}-I_{1} \frac{\Delta M_{2}}{\Delta \ddot{\theta}_{2}}-I_{2} \frac{\Delta M_{1}}{\Delta \ddot{\theta}_{1}}-\frac{\Delta M_{1}}{\Delta \ddot{\theta}_{2}} \frac{\Delta M_{2}}{\Delta \ddot{\theta}_{1}}+\frac{\Delta M_{2}}{\Delta \ddot{\theta}_{2}} \frac{\Delta M_{1}}{\Delta \ddot{\theta}_{1}}}
\end{array}\right.
$$

The error can now be propagated into previous equation.

Therefore, the following is used:

$$
\left|\Delta \ddot{\theta}_{2}^{b} \cdot \Delta \ddot{\theta}_{1}^{a}-\Delta \ddot{\theta}_{2}^{a} \cdot \Delta \ddot{\theta}_{1}^{b}\right| \leq \max _{i=1,2}\left(\left(\Delta \ddot{\theta}_{i}^{b}\right)^{2}+\left(\Delta \ddot{\theta}_{i}^{a}\right)^{2}\right)
$$

So that

$$
\begin{aligned}
A E\left(M_{i}^{n+1, k}\right)^{2} & =\varepsilon_{1}^{2}=\varepsilon_{1}{ }^{2} \cdot\left(\frac{\left|\Delta \ddot{\theta}_{2}^{b} \cdot \Delta \ddot{\theta}_{1}^{a}-\Delta \ddot{\theta}_{2}^{a} \cdot \Delta \ddot{\theta}_{1}^{b}\right|}{\left|\Delta \ddot{\theta}_{2}^{b} \cdot \Delta \ddot{\theta}_{1}^{a}-\Delta \ddot{\theta}_{2}^{a} \cdot \Delta \ddot{\theta}_{1}^{b}\right|}\right)^{2} \\
& \leq \varepsilon_{1}{ }^{2} \cdot \frac{\max _{i=1,2}\left(\left(\Delta \ddot{\theta}_{i}^{b}\right)^{2}+\left(\Delta \ddot{\theta}_{i}^{a}\right)^{2}\right) \cdot\left|\Delta \ddot{\theta}_{2}^{b} \cdot \Delta \ddot{\theta}_{1}^{a}-\Delta \ddot{\theta}_{2}^{a} \cdot \Delta \ddot{\theta}_{1}^{b}\right|}{\left(\Delta \ddot{\theta}_{2}^{b} \cdot \Delta \ddot{\theta}_{1}^{a}-\Delta \ddot{\theta}_{2}^{a} \cdot \Delta \ddot{\theta}_{1}^{b}\right)^{2}} \\
& =\frac{1}{2} \cdot \max _{i, j}\left(A E\left(\frac{\Delta M_{i}}{\Delta \ddot{\theta}_{j}}\right)\right)^{2} \cdot\left|\Delta \ddot{\theta}_{2}^{b} \cdot \Delta \ddot{\theta}_{1}^{a}-\Delta \ddot{\theta}_{2}^{a} \cdot \Delta \ddot{\theta}_{1}^{b}\right|
\end{aligned}
$$

The error propagation for the relative error of the angular accelerations for the next coupling iteration, with "relative error" defined as the absolute error of the angular accelerations divided by the value of the angular accelerations, then results in: 


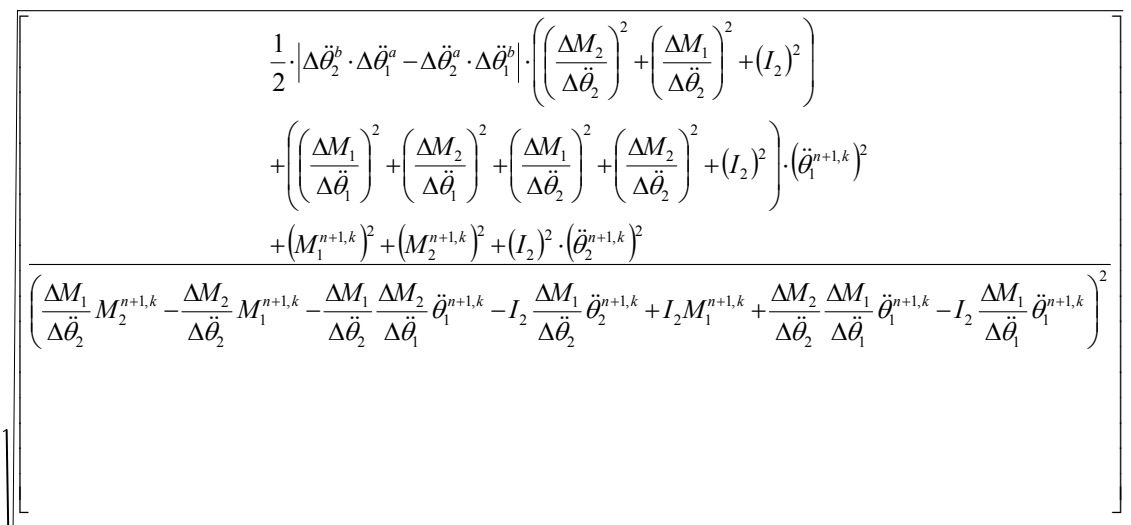

$\left[+\left[\frac{\left(\frac{\Delta M_{2}}{\Delta \ddot{\theta}_{1}}\right)^{2}+\left(\frac{\Delta M_{1}}{\Delta \ddot{\theta}_{2}}\right)^{2}+\left(\frac{\Delta M_{1}}{\Delta \ddot{\theta}_{1}}\right)^{2}+\left(\frac{\Delta M_{2}}{\Delta \ddot{\theta}_{2}}\right)^{2}+\left(I_{2}\right)^{2}+\left(I_{1}\right)^{2}}{\left(I_{1} I_{2}-I_{1} \frac{\Delta M_{2}}{\Delta \ddot{\theta}_{2}}-I_{2} \frac{\Delta M_{1}}{\Delta \ddot{\theta}_{1}}-\frac{\Delta M_{1}}{\Delta \ddot{\theta}_{2}} \frac{\Delta M_{2}}{\Delta \ddot{\theta}_{1}}+\frac{\Delta M_{2}}{\Delta \ddot{\theta}_{2}} \frac{\Delta M_{1}}{\Delta \ddot{\theta}_{1}}\right)^{2}}\right]\right.$

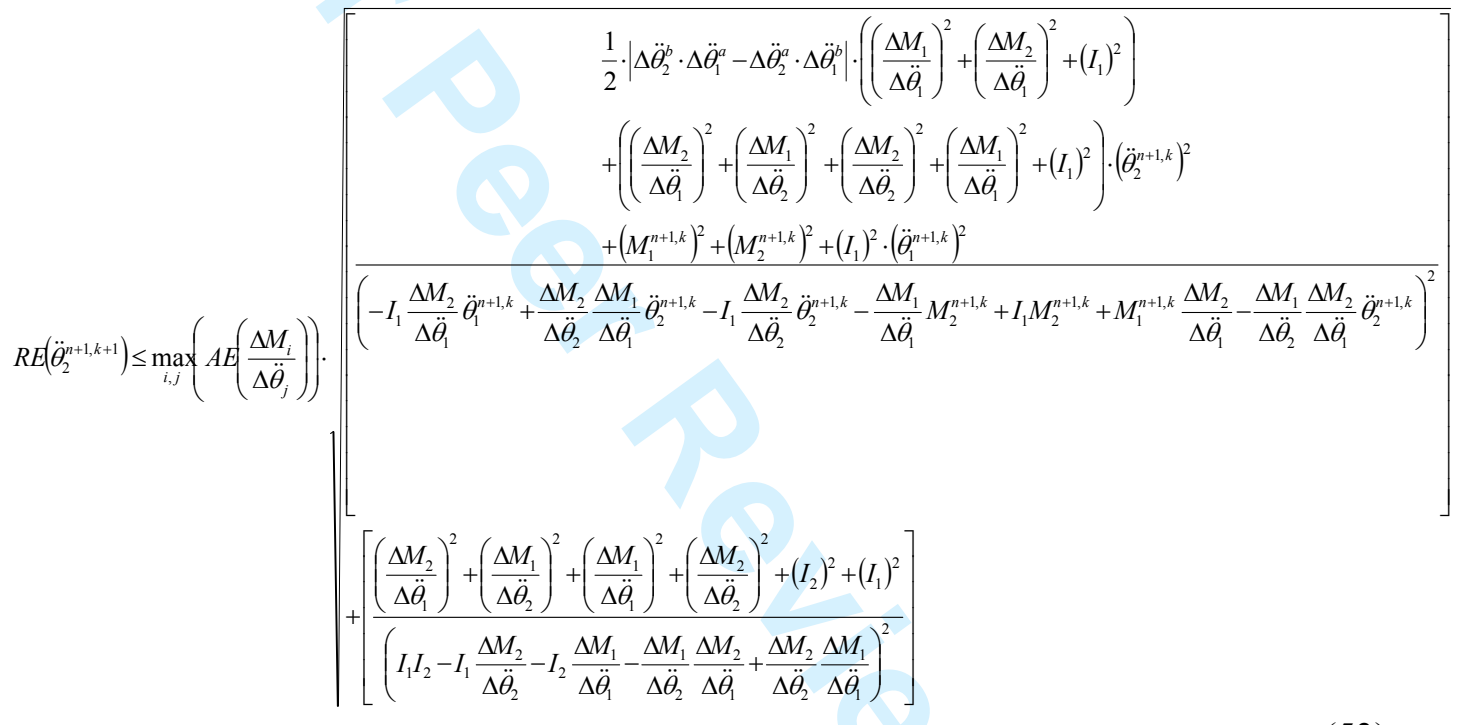

The root terms at the right-hand side of previous equation can be simplified in notation as the factors $f_{1}$ and $f_{2}$ :

$$
\begin{aligned}
& R E\left(\ddot{\theta}_{1}^{n+1, k+1}\right)=\frac{A E\left(\ddot{\theta}_{1}^{n+1, k+1}\right)}{\ddot{\theta}_{1}^{n+1, k+1}} \leq \max _{i, j}\left(A E\left(\frac{\Delta M_{i}}{\Delta \ddot{\theta}_{j}}\right)\right) \cdot f_{1}\left(\frac{\Delta M_{1}}{\Delta \ddot{\theta}_{1}}, \frac{\Delta M_{1}}{\Delta \ddot{\theta}_{2}}, \frac{\Delta M_{2}}{\Delta \ddot{\theta}_{1}}, \frac{\Delta M_{2}}{\Delta \ddot{\theta}_{2}}, I_{1}, I_{2}, M_{1}^{n+1, k}, M_{2}^{n+1, k}, \ddot{\theta}_{1}^{n+1, k}, \ddot{\theta}_{2}^{n+1, k}\right) \\
& \left.\operatorname{RE}\left(\ddot{\theta}_{2}^{n+1, k+1}\right)=\frac{A E\left(\ddot{\theta}_{2}^{n+1, k+1}\right)}{\ddot{\theta}_{2}^{n+1, k+1}} \leq \max _{i, j} A E\left(\frac{\Delta M_{i}}{\Delta \ddot{\theta}_{j}}\right)\right) \cdot f_{2}\left(\frac{\Delta M_{1}}{\Delta \ddot{\theta}_{1}}, \frac{\Delta M_{1}}{\Delta \ddot{\theta}_{2}}, \frac{\Delta M_{2}}{\Delta \ddot{\theta}_{1}}, \frac{\Delta M_{2}}{\Delta \ddot{\theta}_{2}}, I_{1}, I_{2}, M_{1}^{n+1, k}, M_{2}^{n+1, k}, \ddot{\theta}_{1}^{n+1, k}, \ddot{\theta}_{2}^{n+1, k}\right)
\end{aligned}
$$

From these two equations, the Jacobian update threshold $\varepsilon_{2}$ can now be deduced, based on which it will be decided whether or not to update the Jacobian. The Jacobian will be updated when the relative error of the calculation of the angular accelerations for the next coupling iteration is smaller than this threshold $\varepsilon_{2}$. Thus,

$$
\begin{aligned}
& \operatorname{RE}\left(\ddot{\theta}_{1}^{n+1, k+1}\right) \leq \varepsilon_{2} \\
& \Leftrightarrow \max _{i, j}\left(A E\left(\frac{\Delta M_{i}}{\Delta \ddot{\theta}_{j}}\right)\right) \cdot f_{1}\left(\frac{\Delta M_{1}}{\Delta \ddot{\theta}_{1}}, \frac{\Delta M_{1}}{\Delta \ddot{\theta}_{2}}, \frac{\Delta M_{2}}{\Delta \ddot{\theta}_{1}}, \frac{\Delta M_{2}}{\Delta \ddot{\theta}_{2}}, I_{1}, I_{2}, M_{1}^{n+1, k}, M_{2}^{n+1, k}, \ddot{\theta}_{1}^{n+1, k}, \ddot{\theta}_{2}^{n+1, k}\right) \leq \varepsilon_{2}
\end{aligned}
$$

and 


$$
\begin{aligned}
& \operatorname{RE}\left(\ddot{\theta}_{2}^{n+1, k+1}\right) \leq \varepsilon_{2} \\
& \Leftrightarrow \max _{i, j}\left(A E\left(\frac{\Delta M_{i}}{\Delta \ddot{\theta}_{j}}\right)\right) \cdot f_{2}\left(\frac{\Delta M_{1}}{\Delta \ddot{\theta}_{1}}, \frac{\Delta M_{1}}{\Delta \ddot{\theta}_{2}}, \frac{\Delta M_{2}}{\Delta \ddot{\theta}_{1}}, \frac{\Delta M_{2}}{\Delta \ddot{\theta}_{2}}, I_{1}, I_{2}, M_{1}^{n+1, k}, M_{2}^{n+1, k}, \ddot{\theta}_{1}^{n+1, k}, \ddot{\theta}_{2}^{n+1, k}\right) \leq \varepsilon_{2}
\end{aligned}
$$

with

$$
\max _{i, j}\left(A E\left(\frac{\Delta M_{i}}{\Delta \ddot{\theta}_{j}}\right)\right)=\sqrt{2} \cdot \varepsilon_{1} \cdot \frac{\max _{i=1,2}\left(\sqrt{\left(\Delta \ddot{\theta}_{i}^{a}\right)^{2}+\left(\Delta \ddot{\theta}_{i}^{b}\right)^{2}}\right)}{\left|\Delta \ddot{\theta}_{2}^{b} \cdot \Delta \ddot{\theta}_{1}^{a}-\Delta \ddot{\theta}_{2}^{a} \cdot \Delta \ddot{\theta}_{1}^{b}\right|}
$$

So the criterion is related to the direction and magnitude of the acceleration perturbation vectors, the flow solver convergence threshold $\varepsilon_{1}$ and the factors $f_{1}$ and $f_{2}$.

The factors $f_{1}$ and $f_{2}$ cannot be calculated exactly since they contain terms that are still unkown at that time (for example, the Jacobian components of time step $n+1$ ). However, this problem can be circumvented in two ways.

First, the factors $f_{1}$ and $f_{2}$ can be calculated by using the results of the previous time step (for example the angular accelerations, the moments and the Jacobian components of time step $n$ ), but this raises problems in the first time step (due to the zero-initialisation).

However, such estimation is not necessary since the influence of these factors $f_{1}$ and $f_{2}$ remains (almost) constant in time. This is explained by investigating the order of magnitudes of each of the components in the factors $f_{1}$ and $f_{2}$. From Equation (53), it can be shown that the factors $f_{1}$ and $f_{2}$ are in the order of magnitude of the inverse of the maximal Jacobian component:

$$
f_{i} \sim O\left(\max _{i, j}\left(\frac{\Delta M_{i}}{\Delta \ddot{\theta}_{j}}\right)^{-1}\right)
$$

A sensitivity analysis of this maximum Jacobian component is performed for the two cases described in Section 3.2 and it shows that this maximum value is not varying much in time, since the difference between the highest value (that occurred in an entire time cycle) and the smallest value (that occurred in an entire time cycle) is smaller than one order of magnitude. Therefore, the maximum of the Jacobian components remains approximately constant in time. Furthermore, this maximum can be written as

$$
O\left(\max _{i, j}\left(\frac{\Delta M_{i}}{\Delta \ddot{\theta}_{j}}\right)\right) \approx O\left(I_{i}\right) \cdot \text { Constant }
$$

so that the factors $f_{1}$ and $f_{2}$ can be approximated by

$$
f_{i} \sim O\left(\frac{1}{I_{i}}\right) \cdot \frac{1}{\text { Constant }}
$$

Inserting this in Equation (55) and Equation (56) leads to:

or with Equation (57):

$$
\max _{i, j}\left(A E\left(\frac{\Delta M_{i}}{\Delta \ddot{\theta}_{j}}\right)\right) \cdot O\left(\frac{1}{I_{i}}\right) \cdot \frac{1}{\text { Constant }} \leq \varepsilon_{2}
$$

$$
\frac{\max _{i=1,2}\left(\sqrt{\left(\Delta \ddot{\theta}_{i}^{a}\right)^{2}+\left(\Delta \ddot{\theta}_{i}^{b}\right)^{2}}\right)}{\left|\Delta \ddot{\theta}_{2}^{b} \cdot \Delta \ddot{\theta}_{1}^{a}-\Delta \ddot{\theta}_{2}^{a} \cdot \Delta \ddot{\theta}_{1}^{b}\right|} \cdot \varepsilon_{1} \cdot O\left(\frac{1}{I_{i}}\right) \cdot \frac{\sqrt{2}}{\text { Constant }} \leq \varepsilon_{2}
$$

With a redefinition of the threshold $\varepsilon_{2}$, this can be written as 


$$
\frac{\max _{i=1,2}\left(\sqrt{\left(\Delta \ddot{\theta}_{i}^{a}\right)^{2}+\left(\Delta \ddot{\theta}_{i}^{b}\right)^{2}}\right)}{\left|\Delta \ddot{\theta}_{2}^{b} \cdot \Delta \ddot{\theta}_{1}^{a}-\Delta \ddot{\theta}_{2}^{a} \cdot \Delta \ddot{\theta}_{1}^{b}\right|} \cdot \frac{\varepsilon_{1}}{\min _{i=1,2}\left(I_{i}\right)} \leq \varepsilon_{2}
$$

which is the final form of the Jacobian update criterion, as it is referred to in Section 2.3.2. The appropriate value of the threshold $\varepsilon_{2}$ can then be assessed (and selected) through a sensitivity analysis, as is done in Section 3.4.1.

\section{Appendix 2: Update of the Jacobian}

The update of the Jacobian at coupling iteration $k$ of time step $n+1$, can be categorized into three cases, depending on the acceleration perturbation vectors $\left(\boldsymbol{\Delta} \ddot{\boldsymbol{\theta}}^{a}\right.$ and $\left.\Delta \ddot{\boldsymbol{\theta}}^{b}\right)$ obtained in previous coupling iterations, as is briefly discussed in Section 2.3.2.

\section{Vectors $\Delta \ddot{\theta}^{a}=0$ and $\Delta \ddot{\theta}^{b}=0$}

This means that none of the previous coupling iterations of that time step resulted in useable data to update the Jacobian (i.e. the acceleration perturbation and moment data are equal to zero).

The data generated in the current coupling iteration $k$ will be evaluated on its usability by checking the Jacobian update criterion (Equation (28)). For doing so, the vector $\Delta \ddot{\boldsymbol{\theta}}^{a}$ is set as the difference between the accelerations of coupling iteration $k$ and the accelerations of the reference coupling iteration (i.e. $k=0$ ):

$$
\Delta \ddot{\boldsymbol{\theta}}^{a}=\left[\begin{array}{c}
\Delta \ddot{\theta}_{1}^{n+1, k} \\
\Delta \ddot{\theta}_{2}^{n+1, k}
\end{array}\right]=\left[\begin{array}{l}
\ddot{\theta}_{1}^{n+1, k}-\ddot{\theta}_{1}^{n+1,0} \\
\ddot{\theta}_{2}^{n+1, k}-\ddot{\theta}_{2}^{n+1,0}
\end{array}\right]
$$

For the second vector (i.e. $\Delta \ddot{\boldsymbol{\theta}}^{b}$ ), three possibilities can be distinguished:

(1) $\boldsymbol{\Delta} \ddot{\boldsymbol{\theta}}^{b}=\boldsymbol{\Delta} \ddot{\boldsymbol{\theta}}^{a, n}$, with $\boldsymbol{\Delta} \ddot{\boldsymbol{\theta}}^{a, n}$ the vector $\boldsymbol{\Delta} \ddot{\boldsymbol{\theta}}^{a}$ used in previous time step $n$;

(2) $\Delta \ddot{\boldsymbol{\theta}}^{b}=\boldsymbol{\Delta} \ddot{\boldsymbol{\theta}}^{b, n}$, with $\Delta \ddot{\boldsymbol{\theta}}^{b, n}$ the vector $\Delta \ddot{\boldsymbol{\theta}}^{b}$ used in previous time step $n$;

(3) $\boldsymbol{\Delta} \ddot{\boldsymbol{\theta}}^{b}=\boldsymbol{\Delta} \ddot{\boldsymbol{\theta}}^{a} \perp$, with $\boldsymbol{\Delta} \ddot{\boldsymbol{\theta}}^{a} \perp$ the vector perpendicular to $\Delta \ddot{\boldsymbol{\theta}}^{a}$ :

$$
\left\{\begin{array}{l}
\Delta \ddot{\theta}_{1}^{a \perp}=-\Delta \ddot{\theta}_{2}^{a} \\
\Delta \ddot{\theta}_{2}^{a \perp}=\Delta \ddot{\theta}_{1}^{a}
\end{array}\right.
$$

The moments corresponding with $\Delta \ddot{\boldsymbol{\theta}}^{a \perp}$ are estimated by using the Jacobian of previous coupling iteration $k-1$ :

$$
\left\{\begin{array}{l}
\Delta M_{1}^{a \perp}=\left(\frac{\Delta M_{1}}{\Delta \ddot{\theta}_{1}}\right)_{k-1} \cdot \Delta \ddot{\theta}_{1}^{a \perp}+\left(\frac{\Delta M_{1}}{\Delta \ddot{\theta}_{2}}\right)_{k-1} \cdot \Delta \ddot{\theta}_{2}^{a \perp} \\
\Delta M_{2}^{a \perp}=\left(\frac{\Delta M_{2}}{\Delta \ddot{\theta}_{1}}\right)_{k-1} \cdot \Delta \ddot{\theta}_{1}^{a \perp}+\left(\frac{\Delta M_{2}}{\Delta \ddot{\theta}_{2}}\right)_{k-1} \cdot \Delta \ddot{\theta}_{2}^{a \perp}
\end{array}\right.
$$

The possibility for $\Delta \ddot{\boldsymbol{\theta}}^{b}$ that results in the smallest left-hand side of the Jacobian update criterion (Equation (28)) is chosen.

When this smallest value is smaller than or equal to $\varepsilon_{2}$ (i.e. Equation (28) is fulfilled), then the update of the Jacobian is done by Equation (23), with

$$
\boldsymbol{\Delta} \boldsymbol{M}^{a}=\left[\begin{array}{l}
\Delta M_{1}^{n+1, k} \\
\Delta M_{2}^{n+1, k}
\end{array}\right]=\left[\begin{array}{l}
M_{1}^{n+1, k}-M_{1}^{n+1,0} \\
M_{2}^{n+1, k}-M_{2}^{n+1,0}
\end{array}\right]
$$

and $\boldsymbol{\Delta} \boldsymbol{M}^{\boldsymbol{b}}$ set equal to the moment data corresponding with the chosen $\boldsymbol{\Delta} \ddot{\boldsymbol{\theta}}^{b}$. After this Jacobian update, the vector $\boldsymbol{\Delta} \ddot{\boldsymbol{\theta}}^{b}$ is discarded by setting $\boldsymbol{\Delta} \ddot{\boldsymbol{\theta}}^{b}=\mathbf{0}$ (and $\boldsymbol{\Delta} \boldsymbol{M}^{\boldsymbol{b}}=\mathbf{0}$ ), since 
$\Delta \boldsymbol{M}^{\boldsymbol{b}}$ is not derived from coupling iterations in the current time step and hence does not need to be stored.

However, when the smallest value for the left-hand side is greater than $\varepsilon_{2}$ (i.e. Equation (28) is not fulfilled), the Jacobian will not be updated. Both vectors $\Delta \ddot{\boldsymbol{\theta}}^{a}$ and $\boldsymbol{\Delta} \ddot{\boldsymbol{\theta}}^{b}$ (and their moment data $\Delta \boldsymbol{M}^{\boldsymbol{a}}$ and $\boldsymbol{\Delta} \boldsymbol{M}^{\boldsymbol{b}}$ ) are then discarded by setting them equal to $\mathbf{0}$.

\section{Vector $\Delta \ddot{\theta}^{a} \neq 0$ and vector $\Delta \ddot{\theta}^{b}=0$}

An appropriate vector $\boldsymbol{\Delta} \ddot{\theta}^{a}$ (with moment data $\Delta \boldsymbol{M}^{a}$ ) is already obtained in a previous coupling iteration.

The data generated in this coupling iteration $k$ will first be evaluated on its usability by checking the Jacobian update criterion (Equation (28)). For doing so, the vector $\Delta \ddot{\boldsymbol{\theta}}^{b}$ is set as the difference between the accelerations of coupling iteration $k$ and the accelerations of the reference coupling iteration (i.e. $k=0$ ):

$$
\Delta \ddot{\boldsymbol{\theta}}^{\boldsymbol{b}}=\left[\begin{array}{c}
\Delta \ddot{\theta}_{1}^{n+1, k} \\
\Delta \ddot{\theta}_{2}^{n+1, k}
\end{array}\right]=\left[\begin{array}{l}
\ddot{\theta}_{1}^{n+1, k}-\ddot{\theta}_{1}^{n+1,0} \\
\ddot{\theta}_{2}^{n+1, k}-\ddot{\theta}_{2}^{n+1,0}
\end{array}\right]
$$

Also, the data concerning the moments is set:

$$
\boldsymbol{\Delta} \boldsymbol{M}^{b}=\left[\begin{array}{l}
\Delta M_{1}^{n+1, k} \\
\Delta M_{2}^{n+1, k}
\end{array}\right]=\left[\begin{array}{l}
M_{1}^{n+1, k}-M_{1}^{n+1,0} \\
M_{2}^{n+1, k}-M_{2}^{n+1,0}
\end{array}\right]
$$

When the Jacobian update criterion (Equation (28)) is satisfied, the Jacobian components are updated by solving Equation (23) with $\boldsymbol{\Delta} \ddot{\boldsymbol{\theta}}^{a}$ and $\boldsymbol{\Delta} \ddot{\boldsymbol{\theta}}^{b}$ (and their corresponding moment data $\Delta \boldsymbol{M}^{a}$ and $\boldsymbol{\Delta} \boldsymbol{M}^{b}$ ).

If the Jacobian update criterion is not fulfilled, then the Jacobian is not updated and the vector $\boldsymbol{\Delta} \ddot{\boldsymbol{\theta}}^{\boldsymbol{b}}$ (and its moment data $\boldsymbol{\Delta} \boldsymbol{M}^{\boldsymbol{b}}$ ) is not used. The vector $\boldsymbol{\Delta} \ddot{\boldsymbol{\theta}}^{\boldsymbol{b}}$ is then discarded (i.e. $\boldsymbol{\Delta} \ddot{\boldsymbol{\theta}}^{\boldsymbol{b}}=$ $\mathbf{0}$ and $\left.\boldsymbol{\Delta} \boldsymbol{M}^{\boldsymbol{b}}=\mathbf{0}\right)$.

\section{Vector $\Delta \ddot{\theta}^{a} \neq 0$ and vector $\Delta \ddot{\theta}^{b} \neq 0$}

Two vectors $\boldsymbol{\Delta} \ddot{\boldsymbol{\theta}}^{a}$ and $\boldsymbol{\Delta} \ddot{\boldsymbol{\theta}}^{\boldsymbol{b}}$ (with corresponding $\boldsymbol{\Delta} \boldsymbol{M}^{\boldsymbol{a}}$ and $\boldsymbol{\Delta} \boldsymbol{M}^{\boldsymbol{b}}$ ), appropriate for the update of the Jacobian, have already been generated in previous coupling iterations. So, in a previous coupling iteration, the Jacobian was already updated with that data. Therefore, the Jacobian is kept.

\section{Appendix 3: Acceleration of the new coupling iteration in case of a Jacobian of bad quality}

When the quality of the (last used) Jacobian is insufficient, the angular accelerations of the next coupling iteration $k+1$ are calculated in such a way that it will become possible to calculate a good Jacobian in the next coupling iteration by the acceleration and moment data generated in this next coupling iteration.

In order to calculate such appropriate angular accelerations, three cases can be distinguished, depending on $\boldsymbol{\Delta} \ddot{\boldsymbol{\theta}}^{a}$ and $\boldsymbol{\Delta} \ddot{\boldsymbol{\theta}}^{b}$ (and thus depending on the amount of data that are already acquired in previous coupling iterations), as is briefly described in Section 2.3.4:

\section{Vectors $\Delta \ddot{\theta}^{a}=0$ and $\Delta \ddot{\theta}^{b}=0$}

This means that the current and previous coupling iterations did not resulted in useable data for the update of the Jacobian. So, in the next coupling iteration $k+1$, one of the leaflets will be perturbed with a significant parameter $\delta$, since this will result in an appropriate $\Delta \ddot{\boldsymbol{\theta}}^{a}$ (and 
$\Delta \boldsymbol{M}^{a}$ ) in this next coupling iteration, as is described in (Annerel et al. 2010; Dahl et al. 2010). For example,

$$
\left\{\begin{array} { l } 
{ \Delta \ddot { \theta } _ { 1 } ^ { n + 1 , k + 1 } = \delta } \\
{ \Delta \ddot { \theta } _ { 2 } ^ { n + 1 , k + 1 } = 0 }
\end{array} \Rightarrow \left\{\begin{array}{l}
\ddot{\theta}_{1}^{n+1, k+1}=\ddot{\theta}_{1}^{n+1, k=0}+\delta \\
\ddot{\theta}_{2}^{n+1, k+1}=\ddot{\theta}_{2}^{n+1, k=0}
\end{array}\right.\right.
$$

The method is not sensitive to the exact value of this parameter $\delta$ (Vierendeels et al. 2005). As noted above, it should be chosen so that it is large enough to result in a significant change of the moments (i.e. $\Delta \boldsymbol{M}^{\boldsymbol{a}}$ must become significantly large with respect to the flow solver convergence threshold). However, it should not be chosen too large, because this could result in a failing mesh motion. In the cases simulated in Section $3,|\delta|$ is equal to $10 \mathrm{rad} / \mathrm{s}^{2}$. The mathematical sign of $\delta$ depends on the movement phase (opening or closing) and the specific leaflet (left or right).

\section{Vector $\Delta \ddot{\theta}^{a} \neq 0$ and vector $\Delta \ddot{\theta}^{b}=0$}

The data of one of the current or previous coupling iterations (stored as $\boldsymbol{\Delta} \ddot{\boldsymbol{\theta}}^{a}$ with $\boldsymbol{\Delta} \boldsymbol{M}^{\boldsymbol{a}}$ ) can thus still be used to update the Jacobian in the next coupling iteration.

In order to be sure that the perturbation of the leaflet accelerations in the next coupling iteration will generate data that will result in a good update of the Jacobian (i.e. the data of the next coupling iteration $k+1$ are useable as $\boldsymbol{\Delta} \ddot{\boldsymbol{\theta}}^{\boldsymbol{b}}$ with $\boldsymbol{\Delta} \boldsymbol{M}^{\boldsymbol{b}}$ ), the leaflets are perturbed in a direction that is perpendicular to vector $\Delta \ddot{\boldsymbol{\theta}}^{a}$, for example,

$$
\left\{\begin{array} { l } 
{ \Delta \ddot { \theta } _ { 1 } ^ { n + 1 , k + 1 } = - \Delta \ddot { \theta } _ { 2 } ^ { a } } \\
{ \Delta \ddot { \theta } _ { 2 } ^ { n + 1 , k + 1 } = \Delta \ddot { \theta } _ { 1 } ^ { a } }
\end{array} \Rightarrow \left\{\begin{array}{l}
\ddot{\theta}_{1}^{n+1, k+1}=\ddot{\theta}_{1}^{n+1,0}-\Delta \ddot{\theta}_{2}^{a} \\
\ddot{\theta}_{2}^{n+1, k+1}=\ddot{\theta}_{2}^{n+1,0}+\Delta \ddot{\theta}_{1}^{a}
\end{array}\right.\right.
$$

or

$$
\left\{\begin{array} { l } 
{ \Delta \ddot { \theta } _ { 1 } ^ { n + 1 , k + 1 } = \Delta \ddot { \theta } _ { 2 } ^ { a } } \\
{ \Delta \ddot { \theta } _ { 2 } ^ { n + 1 , k + 1 } = - \Delta \ddot { \theta } _ { 1 } ^ { a } }
\end{array} \Rightarrow \left\{\begin{array}{l}
\ddot{\theta}_{1}^{n+1, k+1}=\ddot{\theta}_{1}^{n+1,0}+\Delta \ddot{\theta}_{2}^{a} \\
\ddot{\theta}_{2}^{n+1, k+1}=\ddot{\theta}_{2}^{n+1,0}-\Delta \ddot{\theta}_{1}^{a}
\end{array}\right.\right.
$$

However, it must be checked whether or not these perturbations exceed the limited position of the leaflets. So, when one of these perpendicular perturbations is blocked, then one of the two leaflets is perturbed with the small parameter $\delta$ :

$$
\left\{\begin{array} { l } 
{ \Delta \ddot { \theta } _ { 1 } ^ { n + 1 , k + 1 } = \delta } \\
{ \Delta \ddot { \theta } _ { 2 } ^ { n + 1 , k + 1 } = 0 }
\end{array} \Rightarrow \left\{\begin{array}{l}
\ddot{\theta}_{1}^{n+1, k+1}=\ddot{\theta}_{1}^{n+1,0}+\delta \\
\ddot{\theta}_{2}^{n+1, k+1}=\ddot{\theta}_{2}^{n+1,0}
\end{array}\right.\right.
$$

or

$$
\left\{\begin{array} { l } 
{ \Delta \ddot { \theta } _ { 1 } ^ { n + 1 , k + 1 } = 0 } \\
{ \Delta \ddot { \theta } _ { 2 } ^ { n + 1 , k + 1 } = \delta }
\end{array} \Rightarrow \left\{\begin{array}{l}
\ddot{\theta}_{1}^{n+1, k+1}=\ddot{\theta}_{1}^{n+1,0} \\
\ddot{\theta}_{2}^{n+1, k+1}=\ddot{\theta}_{2}^{n+1,0}+\delta
\end{array}\right.\right.
$$

depending on which of the two perturbations is resulting in the smallest left-hand side of the Jacobian update criterion (Equation (28)).

\section{Vector $\Delta \ddot{\theta}^{a} \neq 0$ and vector $\Delta \ddot{\theta}^{b} \neq 0$}

No extra data are needed, since all the data that are used to update the Jacobian, depend already on time step $n+1$. The slow residual drop rate is hence inherent to the problem and cannot be resolved efficiently by generating extra data. The Jacobian is thus kept. The angular accelerations of the next coupling iteration are thus calculated by Equation (17) with the Jacobian and this next coupling iteration is subsequently initiated. 


\section{Table captions}

Table 1. Influence of FSI convergence threshold on the performance of the quasiNewton and the Aitken relaxation method, with $\varepsilon_{1}=\varepsilon_{0} / 100$ and $\varepsilon_{2}=0.001$.

Table 2. Variations of $\varepsilon_{2}$ with $\varepsilon_{1}$ fixed to $\varepsilon_{0} / 100$ and $\varepsilon_{0}$ equal to $1 \mathrm{E}-13 \mathrm{Nm}$, for the Valsalva sinuses geometry, with the quasi-Newton method.

Table 3. Variations of $\varepsilon_{1}$ with $\varepsilon_{2}$ fixed to 0.001 and $\varepsilon_{0}$ equal to $1 \mathrm{E}-13 \mathrm{Nm}$, for the Valsalva sinuses geometry, with the quasi-Newton method.

Table 4. Variations of $\varepsilon_{1}$ and $\varepsilon_{2}$ with $\varepsilon_{0}$ equal to $1 \mathrm{E}-10 \mathrm{Nm}$, for the Valsalva sinuses geometry, with the quasi-Newton method.

\section{Figure captions}

Figure 1. Simplified flow chart of a FSI coupling algorithm with two degrees of freedom. $n=$ time step, $k=$ coupling iteration step, $i=$ leaflet number.

Figure 2. Flow chart of the new quasi-Newton coupling algorithm with two degrees of freedom. $n=$ time step, $k=$ coupling iteration step, $i=$ leaflet number.

Figure 3. View on the first geometry, with straight tube downstream of the valve: seen isometric (left), from front side $(u p)$, from top side (middle) and from inlet (down).

Figure 4. View on the second geometry, with Valsalva sinuses downstream of the valve: seen isometric (left), from front side (up), from top side (middle) and from inlet (down).

Figure 5. Angular position of the leaflets (relative to maximal opening angle) and the aortic flow pulse velocity. The left and right leaflet of the straight tube geometry move symmetrically.

Figure 6. Velocity Magnitude Contours in $\mathrm{m} / \mathrm{s}$ for the straight tube geometry (left) and the Valsalva sinuses geometry (right), visualized on a longitudinal section perpendicular to the leaflet rotation axes, at $t=0.025 \mathrm{~s}(a), t=0.125 \mathrm{~s}(b), t=0.250 \mathrm{~s}$ $(c)$ and $t=0.375 \mathrm{~s}(d)$, represented by respectively the purple, red, green and blue dot in Figure 5.

Figure 7. Needed number of coupling iterations for a time step, as a function of the fixed relaxation factor value and the leaflet position.

Figure 8. Range of the used Aitken relaxation factor values as a function of the angular leaflet position (relative to the fully open position), for the straight tube geometry.

Figure 9. Comparison between the convergence process of quasi-Newton and Aitken relaxation, with $\varepsilon_{0}=1 \mathrm{E}-11 \mathrm{Nm}$ : logarithmic plot of the absolute values of the residuals for the left leaflet, at ten subsequent time steps (between $t=0.02 \mathrm{~s}$ and $t=0.021 \mathrm{~s}$ ). 
Figure 10. Comparison between the convergence process of quasi-Newton and Aitken relaxation, with $\varepsilon_{0}=1 \mathrm{E}-13 \mathrm{Nm}$ : logarithmic plot of the absolute values of the residuals for the left leaflet, at ten subsequent time steps (between $t=0.02 \mathrm{~s}$ and $t=0.021 \mathrm{~s}$ ).

Figure 11. Angular leaflet position (a), angular leaflet velocity $(b)$ and angular leaflet acceleration $(c)$ during the opening phase (left: $t=0 \mathrm{~s} . .0 .05 \mathrm{~s}$; right: $t=0 \mathrm{~s} . .0 .01 \mathrm{~s})$ for strong and loose coupling. Quasi-Newton $(-)$, loose $\omega_{i}^{n+1}=0.1(-\cdots)$, loose $\omega_{i}^{n+1}=0.25$ $(--)$, loose $\omega_{i}^{n+1}=0.325(\cdots)$.

Figure 12. Plots of the pressure field in $\mathrm{Pa}$ (left), velocity field in $\mathrm{m} / \mathrm{s}$ (middle left), a zoom of the velocity field (middle right) and the velocity vectors at the b-datum gap (right) for loose coupling $\left(\omega_{i}^{n+1}=0.325\right)$, visualized on a longitudinal section perpendicular to the leaflet rotation axes, taken at subsequent time steps $(t=0.004 \mathrm{~s}$ $(a), t=0.0045 \mathrm{~s}(b), t=0.005 \mathrm{~s}(c), t=0.0055 \mathrm{~s}(d), t=0.006 \mathrm{~s}(e)$ and $t=0.0065 \mathrm{~s}(f))$.

Figure 13. Plots of the pressure field in Pa (left), velocity field in $\mathrm{m} / \mathrm{s}$ (middle-left), zoom of the velocity field (middle-right) and the velocity vectors at the b-datum gap (right) for strong coupling (quasi-Newton), visualized on a longitudinal section perpendicular to the leaflet rotation axes, taken at subsequent time steps $(t=0.004 \mathrm{~s}$ $(a), t=0.0045 \mathrm{~s}(b), t=0.005 \mathrm{~s}(c), t=0.0055 \mathrm{~s}(d), t=0.006 \mathrm{~s}(e)$ and $t=0.0065 \mathrm{~s}(f))$. 


\begin{tabular}{|c|c|c|c|c|c|c|}
\hline \multirow{2}{*}{$\begin{array}{l}\text { FSI convergence } \\
\text { threshold } \varepsilon_{0} \\
\text { VALSALVA } \\
\text { SINUSES }\end{array}$} & \multirow[t]{2}{*}{ Method } & \multicolumn{5}{|c|}{ Averaged needed number of coupling iterations per time step } \\
\hline & & $\begin{array}{c}\text { Opening } \\
(t=0 s . . \\
0.0725 s)\end{array}$ & $\begin{array}{c}\text { Open } \\
(\mathrm{t}=\mathbf{0 . 0 7 2 5 s} . . \\
\mathbf{0 . 3 2 5 s})\end{array}$ & $\begin{array}{c}\text { Closing } \\
(\mathrm{t}=\mathbf{0 . 3 2 5 s} . . \\
0.38 \mathrm{~s})\end{array}$ & $\begin{array}{c}\text { Closed } \\
(\mathrm{t}=\mathbf{0 . 3 8 \mathrm { s } . .} \text { 1s })\end{array}$ & $\begin{array}{c}\text { Total } \\
(\mathrm{t}=0 \mathrm{0} \text {.. 1s) }\end{array}$ \\
\hline \multirow{2}{*}{$\begin{array}{l}\text { 1E-10Nm } \\
\left(\sim M_{\text {ref }} / 10\right)\end{array}$} & Aitken & 4.411 & 3.953 & 4.957 & 1 & 2.936 \\
\hline & quasi-Newton & 3.573 & 3.304 & 3.687 & 1 & 2.415 \\
\hline \multirow{2}{*}{$\begin{array}{l}\text { 1E-11Nm } \\
\left(\sim M_{\mathrm{ref}} / 100\right)\end{array}$} & Aitken & 4.925 & 4.286 & 5.415 & 1 & 3.180 \\
\hline & quasi-Newton & 3.921 & 3.577 & 3.909 & 1 & 2.571 \\
\hline \multirow{2}{*}{$\begin{array}{c}\mathbf{1 E - 1 2 N m} \\
\left(\sim M_{\mathrm{ref}} / 1000\right)\end{array}$} & Aitken & 5.598 & 4.988 & 5.772 & 1 & 3.499 \\
\hline & quasi-Newton & 4.051 & 3.617 & 4.009 & 1 & 2.621 \\
\hline \multirow{2}{*}{$\begin{array}{c}\text { 1E-13Nm } \\
\left(\sim M_{\mathrm{ref}} / 10000\right)\end{array}$} & Aitken & 6.055 & 5.857 & 6.060 & 1 & 3.795 \\
\hline & quasi-Newton & 4.109 & 3.643 & 4.104 & 1 & 2.656 \\
\hline \multirow{3}{*}{$\begin{array}{c}\mathbf{1 E - 1 4 N m} \\
\left(\sim M_{\mathrm{ref}} / 100000\right) \\
\end{array}$} & Aitken & 6.229 & 6.012 & 6.474 & 1 & 3.936 \\
\hline & quasi-Newton & 4.158 & 3.893 & 4.154 & 1 & 2.719 \\
\hline & & & & & & \\
\hline $\begin{array}{l}\text { STRAIGHT } \\
\text { TUBE }\end{array}$ & & $\begin{array}{c}\text { Opening } \\
(\mathrm{t}=0 \mathrm{~s} . . \\
0.0725 s)\end{array}$ & $\begin{array}{c}\text { Open } \\
(\mathrm{t}=\mathbf{0 . 0 7 2 5 s} . . \\
0.325 \mathrm{~s})\end{array}$ & $\begin{array}{c}\text { Closing } \\
(\mathrm{t}=\mathbf{0 . 3 2 5 \mathrm { s }} . . \\
0.3925 \mathrm{~s}) \\
\end{array}$ & $\begin{array}{c}\text { Closed } \\
(\mathrm{t}=\mathbf{0 . 3 9 2 5 \mathrm { s }} . . \\
1 \mathrm{~s})\end{array}$ & $\begin{array}{c}\text { Total } \\
(\mathrm{t}=\mathbf{0} \text { s .. 1s) }\end{array}$ \\
\hline \multirow{2}{*}{$\begin{array}{c}\text { 1E-13Nm } \\
\left(\sim M_{\mathrm{ref}} / 10000\right)\end{array}$} & Aitken & 6.147 & 1 & 6.025 & 1 & 3.152 \\
\hline & quasi-Newton & 4.064 & 1 & 3.984 & 1 & 2.279 \\
\hline
\end{tabular}

Table 1. Influence of FSI convergence threshold on the performance of the quasi-Newton and the Aitken relaxation method, with $\varepsilon_{1}=\varepsilon_{0} / 100$ and $\varepsilon_{2}=0.001$. 


\begin{tabular}{r|c|c|c|c|c}
\hline Value of $\boldsymbol{\varepsilon}_{\mathbf{2}}$ & $\begin{array}{c}\text { Total } \\
\text { number of } \\
\text { coupling } \\
\text { iterations }\end{array}$ & $\begin{array}{c}\text { Total } \\
\text { number of } \\
\text { time steps }\end{array}$ & $\begin{array}{c}\text { Averaged } \\
\text { needed number } \\
\text { of coupl. it. per } \\
\text { time step }\end{array}$ & $\begin{array}{c}\text { Wall-clock time } \\
\text { per coupling } \\
\text { iteration } \\
\text { (in min) }\end{array}$ & $\begin{array}{c}\text { Wall-clock time } \\
\text { per time step } \\
\text { (in min) }\end{array}$ \\
\hline 0.00001 & 830 & 177 & 4.689 & 1.107 & 5.189 \\
0.0001 & 792 & 177 & 4.475 & 1.130 & 5.057 \\
0.001 & 724 & 177 & 4.090 & 1.105 & 4.522 \\
0.01 & DIVERGENCE & DIVERGENCE & DIVERGENCE & DIVERGENCE & DIVERGENCE \\
0.1 & DIVERGENCE & DIVERGENCE & DIVERGENCE & DIVERGENCE & DIVERGENCE \\
1 & DIVERGENCE & DIVERGENCE & DIVERGENCE & DIVERGENCE & DIVERGENCE \\
\hline
\end{tabular}

Table 2. Variations of $\varepsilon_{2}$ with $\varepsilon_{1}$ fixed to $\varepsilon_{0} / 100$ and $\varepsilon_{0}$ equal to $1 \mathrm{E}-13 \mathrm{Nm}$, for the Valsalva sinuses geometry, with the quasi-Newton method. 


\begin{tabular}{r|c|c|c|c|c}
\hline $\begin{array}{c}\text { Value of } \\
\boldsymbol{\varepsilon}_{1} / \boldsymbol{\varepsilon}_{\mathbf{0}}\end{array}$ & $\begin{array}{c}\text { Total } \\
\text { number of } \\
\text { coupling } \\
\text { iterations }\end{array}$ & $\begin{array}{c}\text { Total } \\
\text { number of } \\
\text { time steps }\end{array}$ & $\begin{array}{c}\text { Averaged } \\
\text { needed number } \\
\text { of coupl. it. per } \\
\text { time step }\end{array}$ & $\begin{array}{c}\text { Wall-clock time } \\
\text { per coupling } \\
\text { iteration } \\
\text { (in min) }\end{array}$ & $\begin{array}{c}\text { Wall-clock time } \\
\text { per time step } \\
\text { (in min) }\end{array}$ \\
\hline 1 & 859 & 177 & 4.853 & 0.847 & 4.113 \\
0.1 & 796 & 177 & 4.497 & 0.963 & 4.330 \\
0.01 & 724 & 177 & 4.090 & 1.105 & 4.522 \\
0.001 & 725 & 177 & 4.096 & 1.268 & 5.194 \\
0.0001 & 714 & 177 & 4.034 & 1.450 & 5.847 \\
\hline
\end{tabular}

Table 3. Variations of $\varepsilon_{1}$ with $\varepsilon_{2}$ fixed to 0.001 and $\varepsilon_{0}$ equal to $1 \mathrm{E}-13 \mathrm{Nm}$, for the Valsalva sinuses geometry, with the quasi-Newton method. 


\begin{tabular}{r|c|c|c|c|c|c}
\hline $\begin{array}{c}\text { Value of } \\
\boldsymbol{\varepsilon}_{\mathbf{1}} / \boldsymbol{\varepsilon}_{\mathbf{0}}\end{array}$ & $\begin{array}{c}\text { Value of } \\
\boldsymbol{\varepsilon}_{\mathbf{2}}\end{array}$ & $\begin{array}{c}\text { Total } \\
\text { number of } \\
\text { coupling } \\
\text { iterations }\end{array}$ & $\begin{array}{c}\text { Total } \\
\text { number of } \\
\text { time steps }\end{array}$ & $\begin{array}{c}\text { Averaged } \\
\text { needed } \\
\text { number of } \\
\text { coupl. it. per } \\
\text { time step }\end{array}$ & $\begin{array}{c}\text { Wall-clock } \\
\text { time per } \\
\text { coupling } \\
\text { iteration } \\
\text { (in min) }\end{array}$ & $\begin{array}{c}\text { Wall-clock } \\
\text { time per time } \\
\text { step (in min) }\end{array}$ \\
\hline 0.1 & 0.001 & DIVERGENCE & DIVERGENCE & DIVERGENCE & DIVERGENCE & DIVERGENCE \\
0.001 & 0.001 & 643 & 177 & 3.633 & 0.947 & 3.441 \\
0.0001 & 0.001 & 625 & 177 & 3.531 & 1.069 & 3.774 \\
\hline 0.01 & 0.001 & 642 & 177 & 3.627 & 0.841 & 3.051 \\
\hline 0.01 & 0.01 & 642 & 177 & 3.627 & 0.841 & 3.051 \\
\hline 0.01 & 0.0001 & DIVERGENCE & DIVERGENCE & DIVERGENCE & DIVERGENCE & DIVERGENCE \\
\hline
\end{tabular}

Table 4. Variations of $\varepsilon_{1}$ and $\varepsilon_{2}$ with $\varepsilon_{0}$ equal to $1 \mathrm{E}-10 \mathrm{Nm}$, for the Valsalva sinuses geometry, with the quasi-Newton method. 


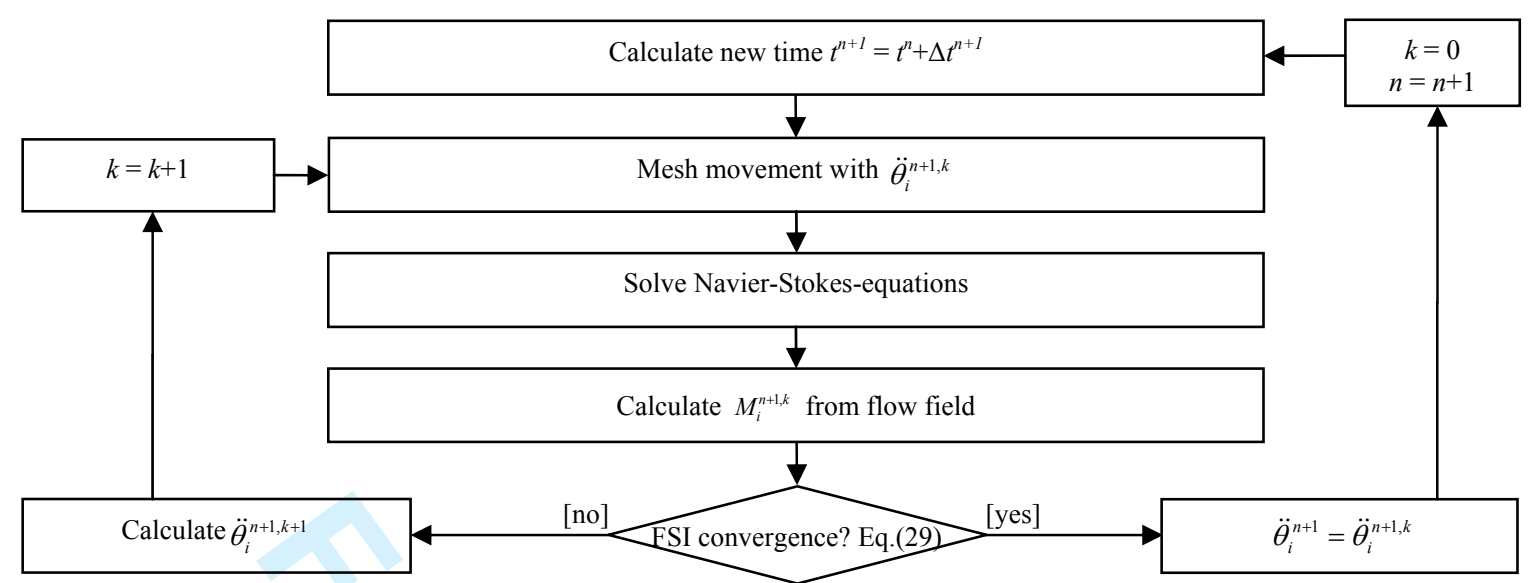

Figure 1. Simplified flow chart of a FSI coupling algorithm with two degrees of freedom. $n=$ time step, $k=$ coupling iteration step, $i=$ leaflet number. 
2

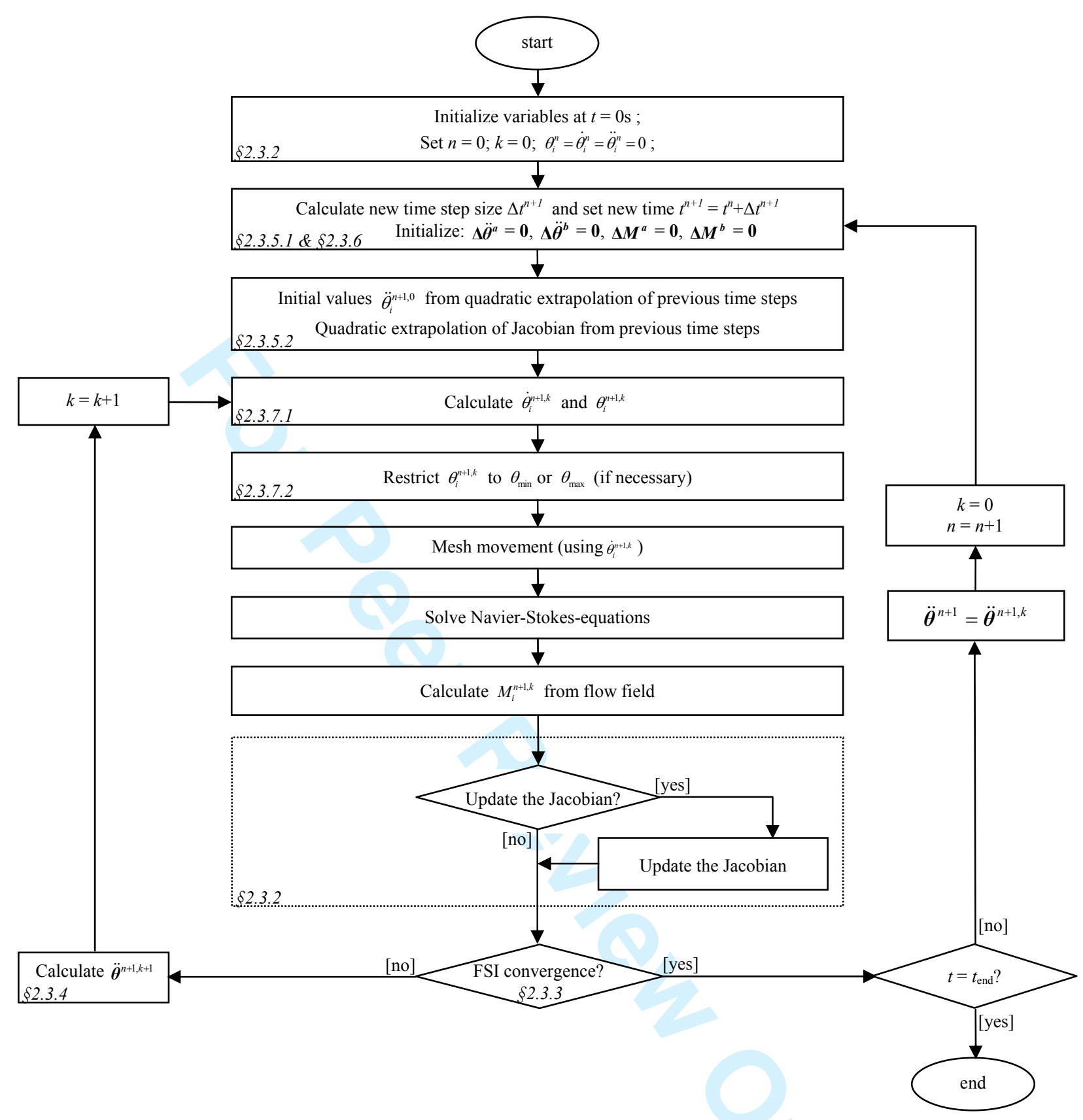

Figure 2. Flow chart of the new quasi-Newton coupling algorithm with two degrees of freedom. $n=$ time step, $k=$ coupling iteration step, $i=$ leaflet number. 


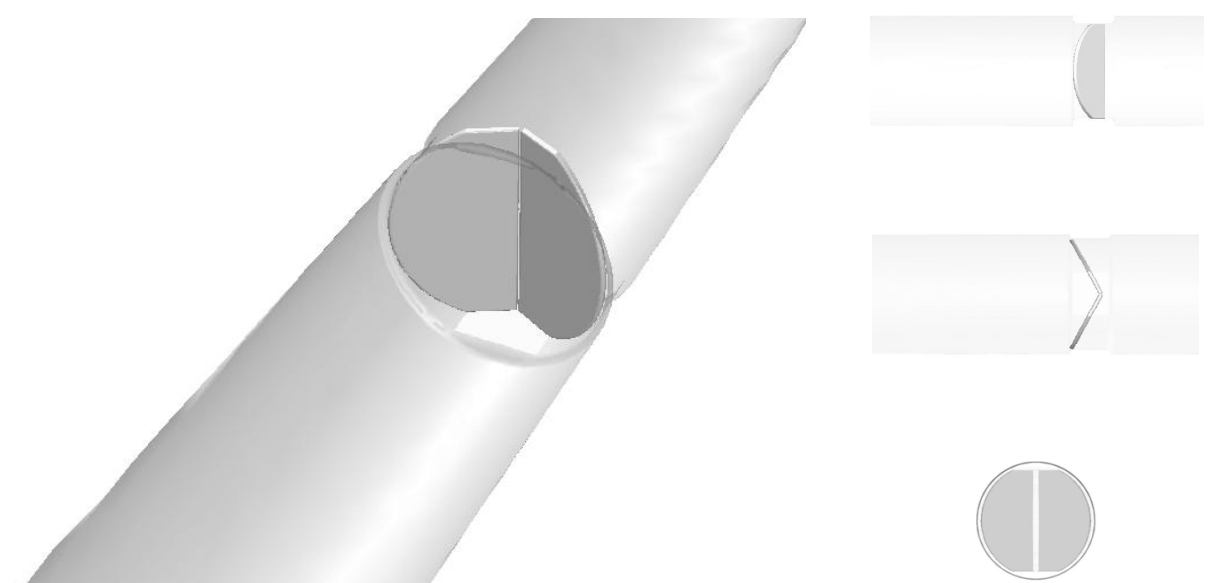

Figure 3. View on the first geometry, with straight tube downstream of the valve: seen isometric (left), from front side (up), from top side (middle) and from inlet (down). 
Figure 4. View on the second geometry, with Valsalva sinuses downstream of the valve: seen isometric (left), from front side (up), from top side (middle) and from inlet (down). 


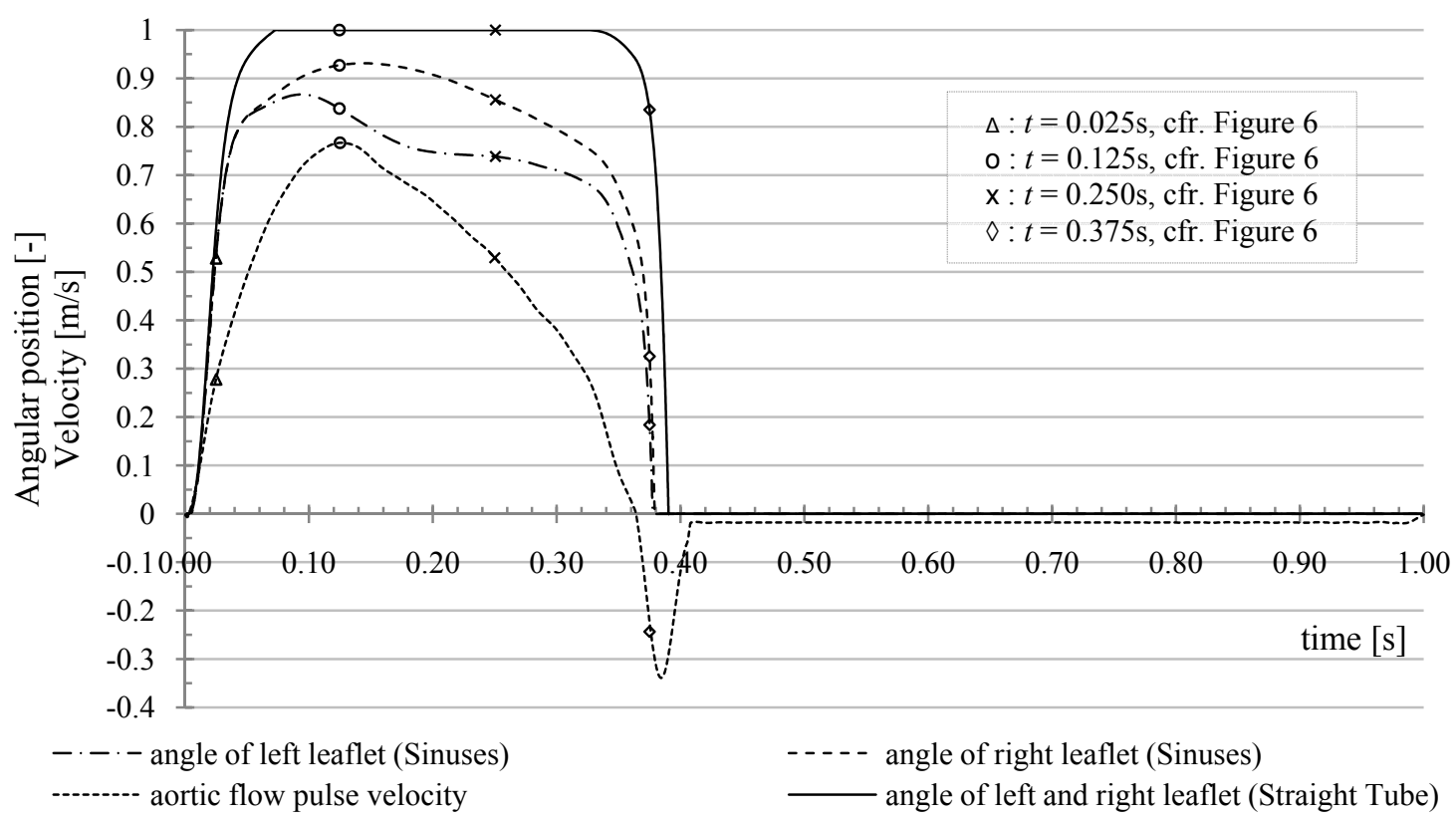

Figure 5. Angular position of the leaflets (relative to maximal opening angle) and the aortic flow pulse velocity. The left and right leaflet of the straight tube geometry move symmetrically. 
(a)
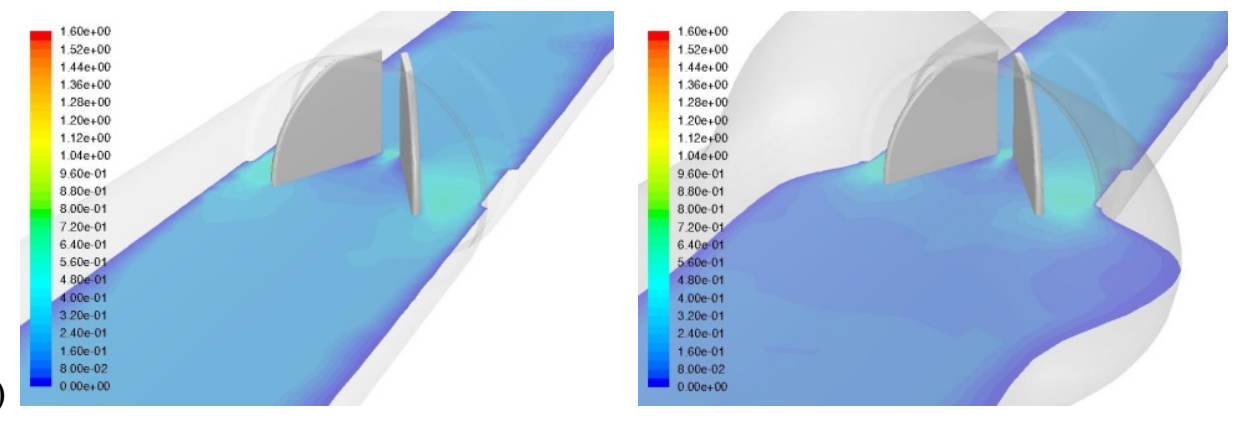

(b)
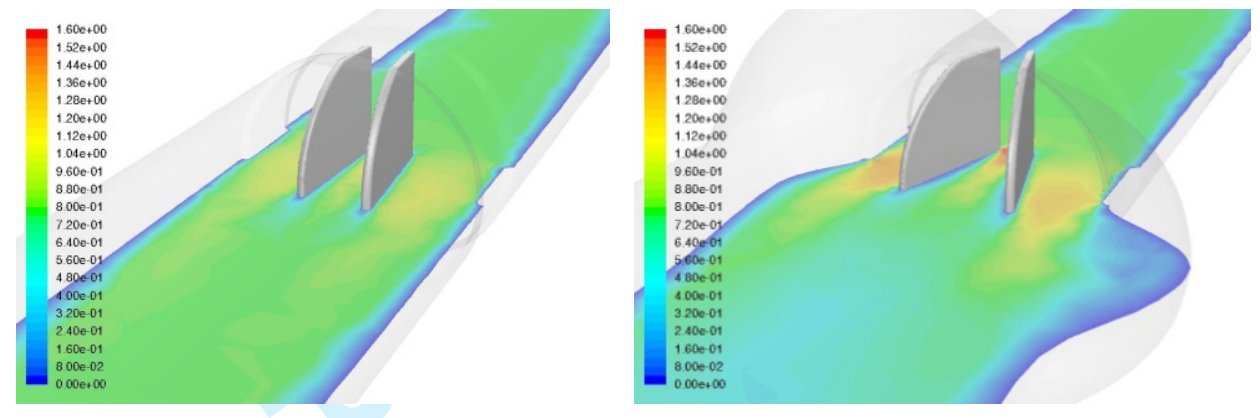

(c)
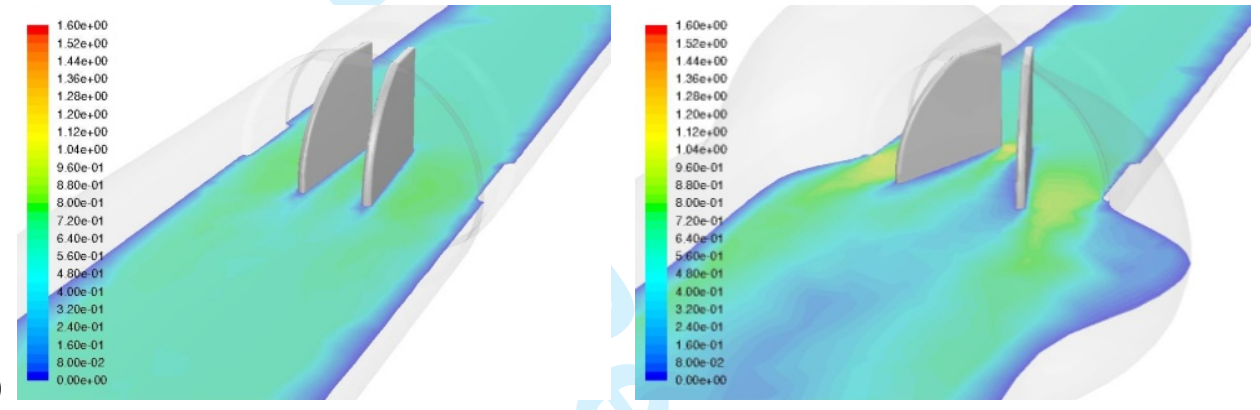

(d)
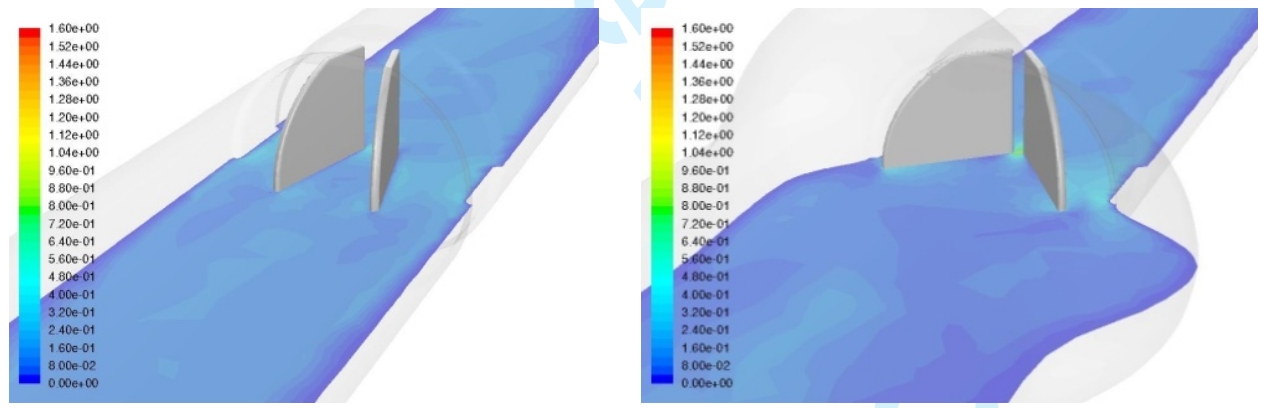

Figure 6. Velocity Magnitude Contours in $\mathrm{m} / \mathrm{s}$ for the straight tube geometry (left) and the Valsalva sinuses geometry (right), visualized on a longitudinal section perpendicular to the leaflet rotation axes, at $t=0.025 \mathrm{~s}(a), t=0.125 \mathrm{~s}(b), t=0.250 \mathrm{~s}(c)$ and $t=0.375 \mathrm{~s}(d)$, represented by respectively the purple, red, green and blue dot in Figure 5. 


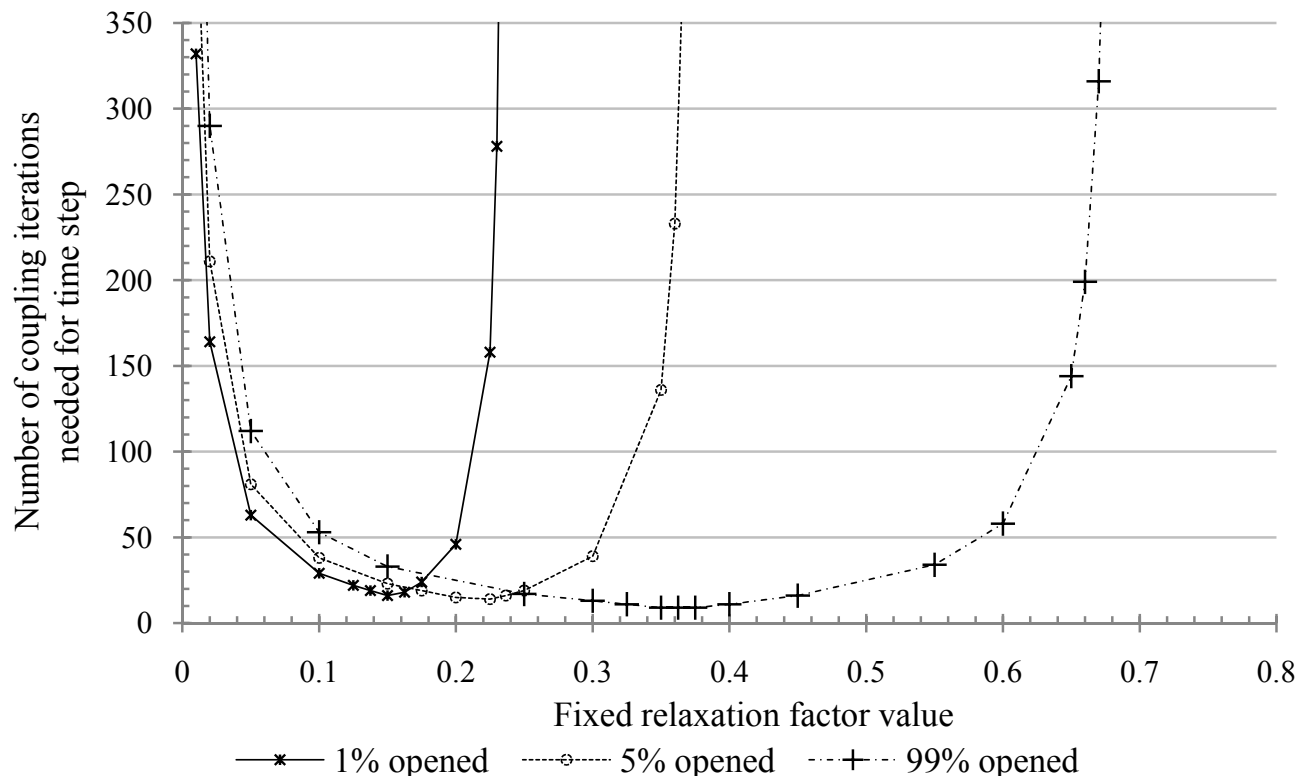

Figure 7. Needed number of coupling iterations for a time step, as a function of the fixed relaxation factor value and the leaflet position. 


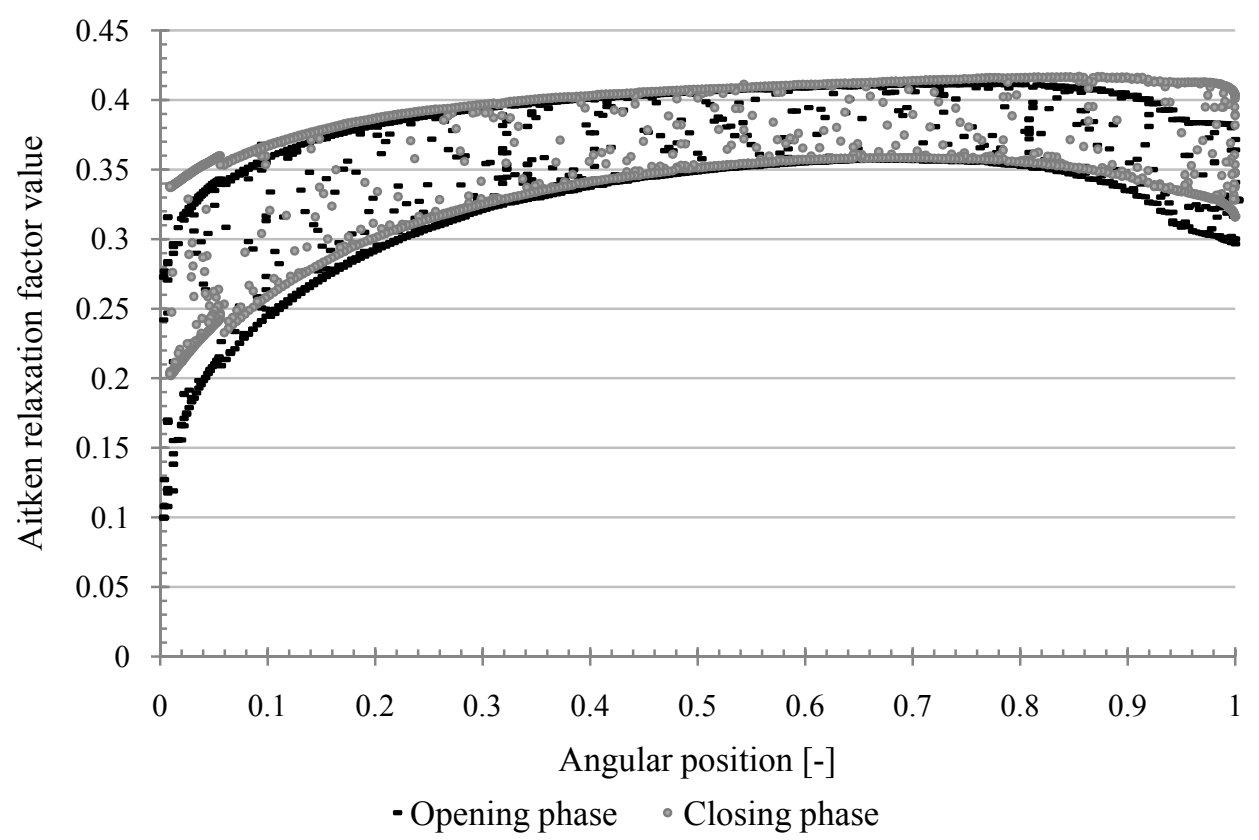

Figure 8. Range of the used Aitken relaxation factor values as a function of the angular leaflet position (relative to the fully open position), for the straight tube geometry. 


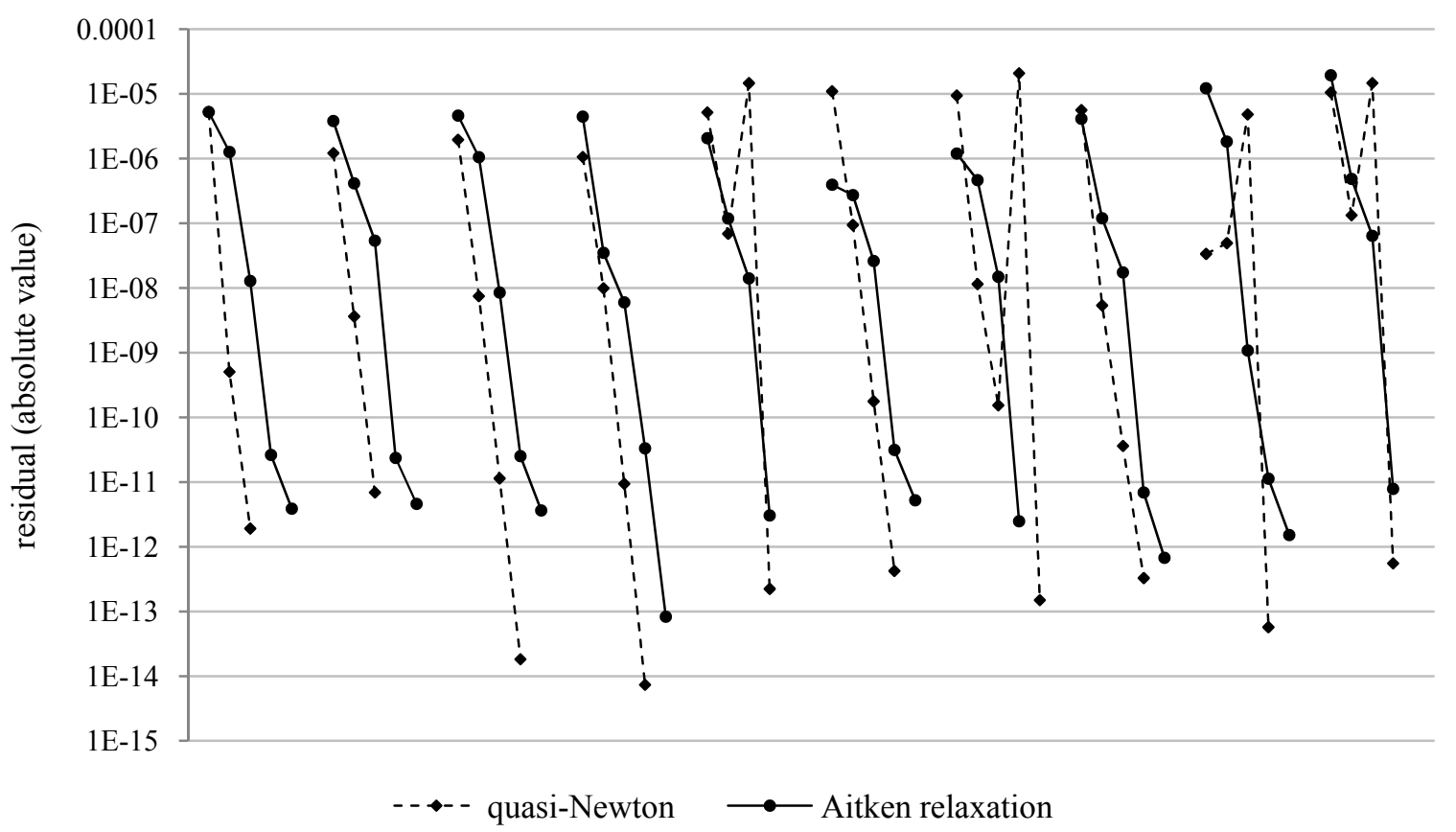

Figure 9. Comparison between the convergence process of quasi-Newton and Aitken relaxation, with $\varepsilon_{0}=1 \mathrm{E}-11 \mathrm{Nm}$ : logarithmic plot of the absolute values of the residuals for the left leaflet, at ten subsequent time steps (between $t=0.02 \mathrm{~s}$ and $t=0.021 \mathrm{~s}$ ). 
Figure 10. Comparison between the convergence process of quasi-Newton and Aitken
relaxation, with $\varepsilon_{0}=1 \mathrm{E}-13 \mathrm{Nm}$ : logarithmic plot of the absolute values of the residuals for the

Figure 10. Comparison between the convergence process of quasi-Newton and Aitken
relaxation, with $\varepsilon_{0}=1 \mathrm{E}-13 \mathrm{Nm}$ : logarithmic plot of the absolute values of the residuals for the left leaflet, at ten subsequent time steps (between $t=0.02 \mathrm{~s}$ and $t=0.021 \mathrm{~s}$ ).

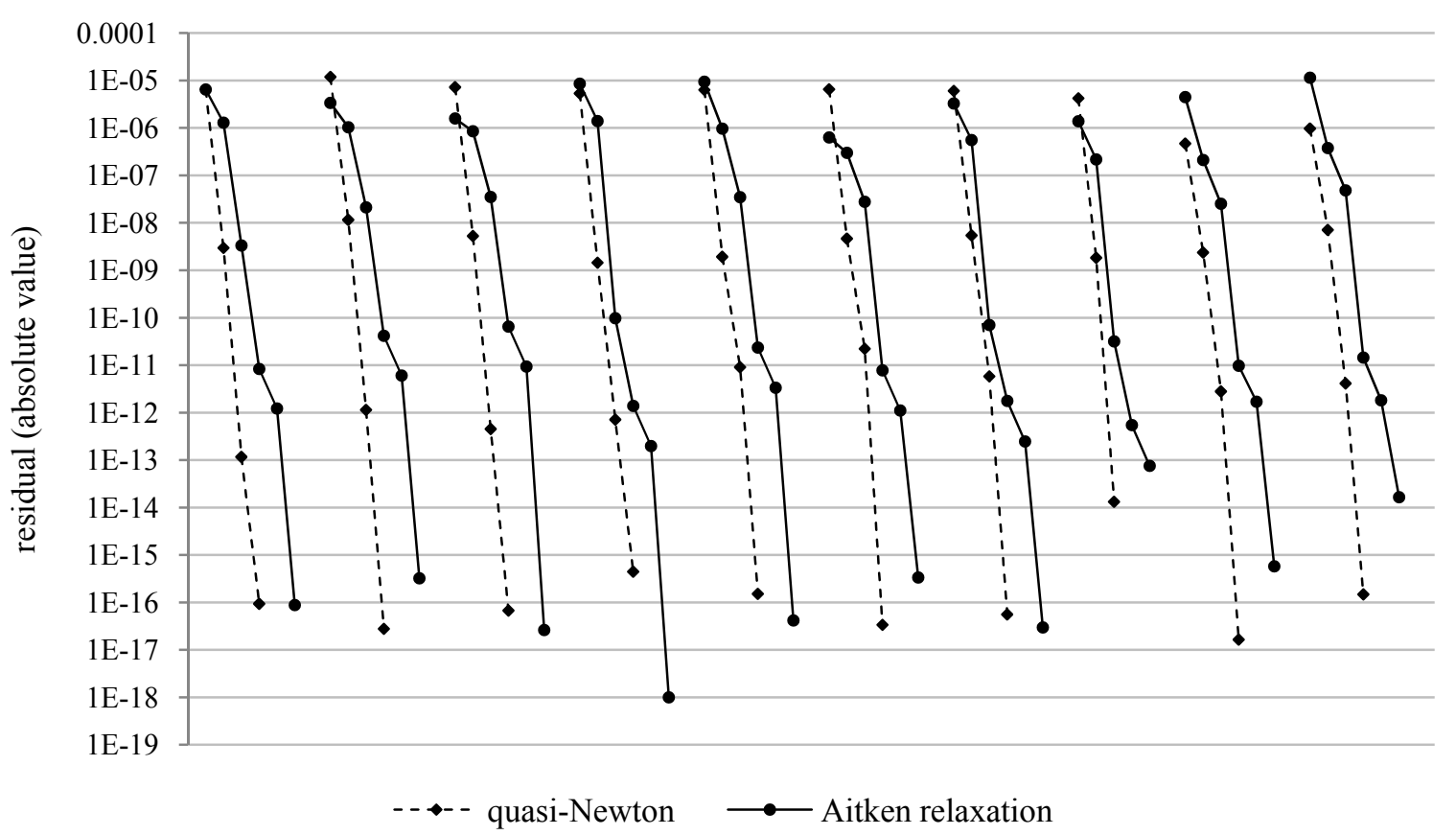


(a)

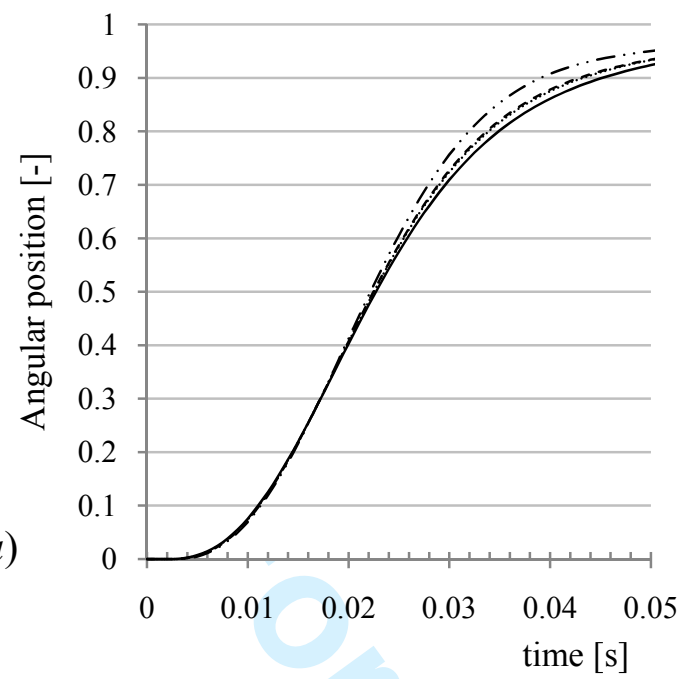

(b)

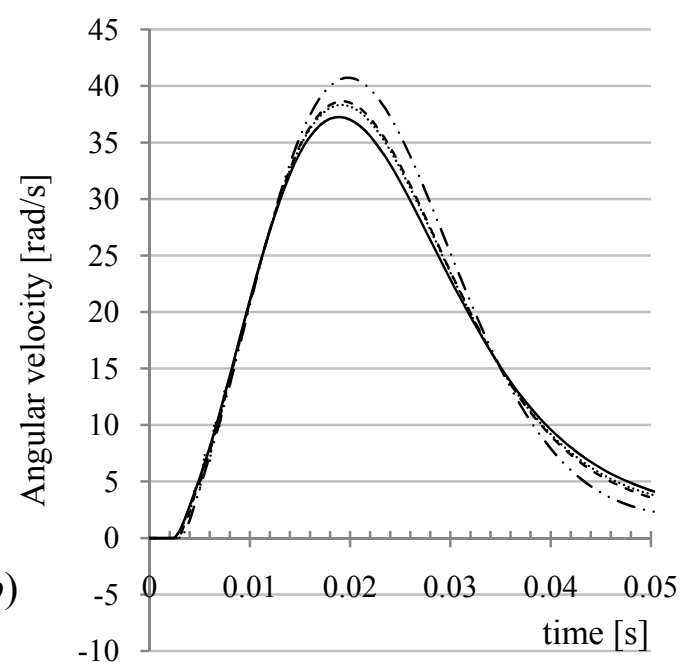

(c)

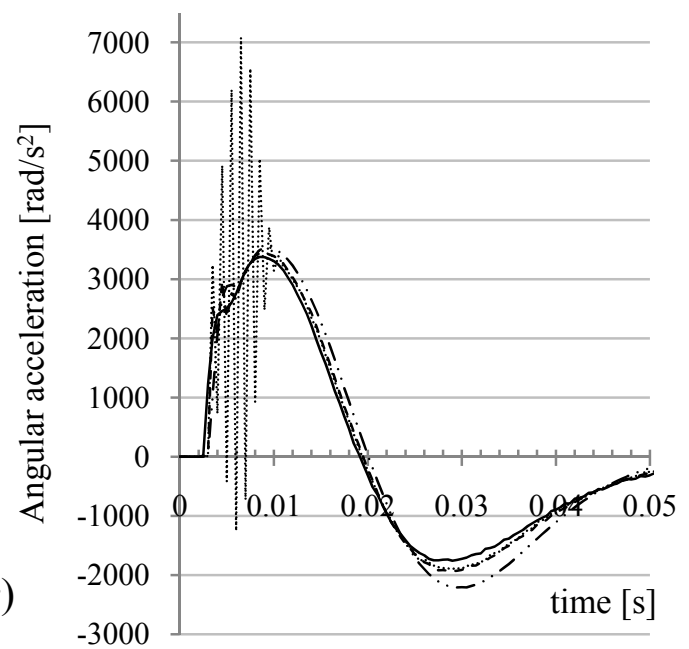

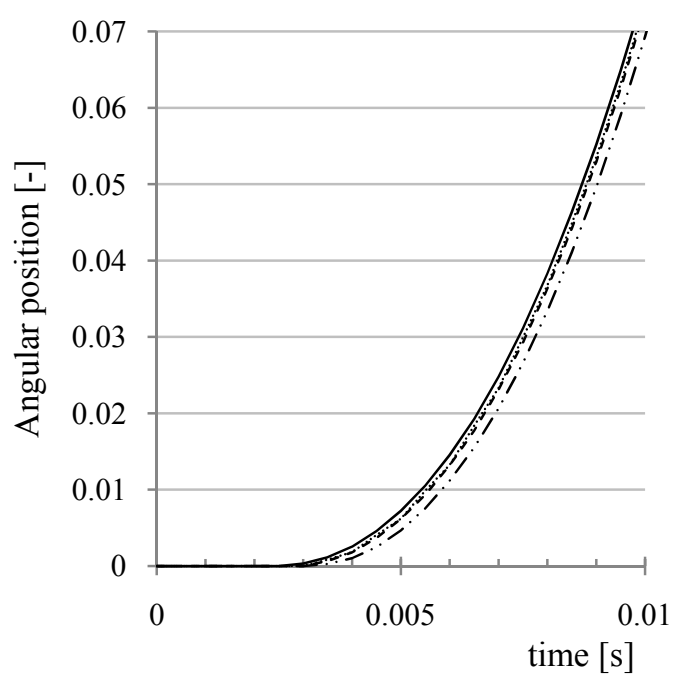
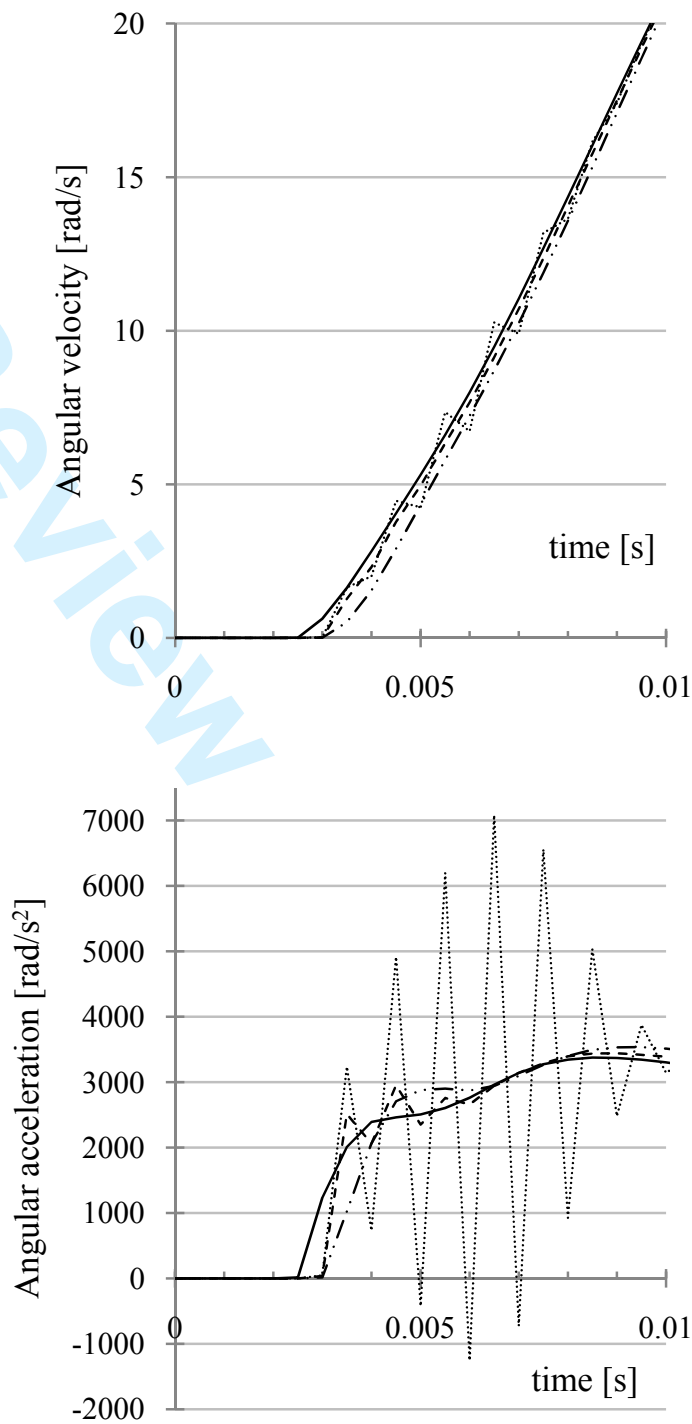

Figure 11. Angular leaflet position ( $a$ ), angular leaflet velocity $(b)$ and angular leaflet acceleration (c) during the opening phase (left: $t=0 \mathrm{~s} . .0 .05 \mathrm{~s}$; right: $t=0 \mathrm{~s} . .0 .01 \mathrm{~s})$ for strong and loose coupling. Quasi-Newton (-), loose $\omega_{i}^{n+1}=0.1(-\cdots)$, loose $\omega_{i}^{n+1}=0.25(---)$, loose $\omega_{i}^{n+1}=0.325(\cdots)$. 
(a)
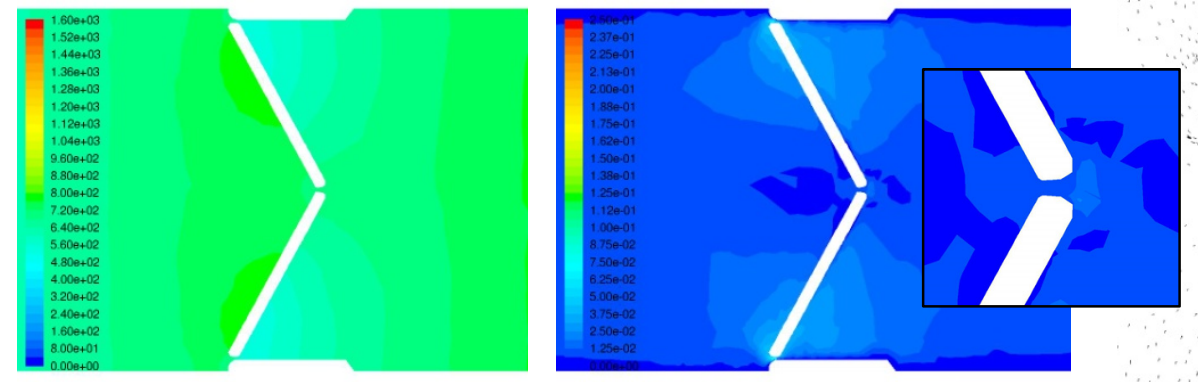

(b)
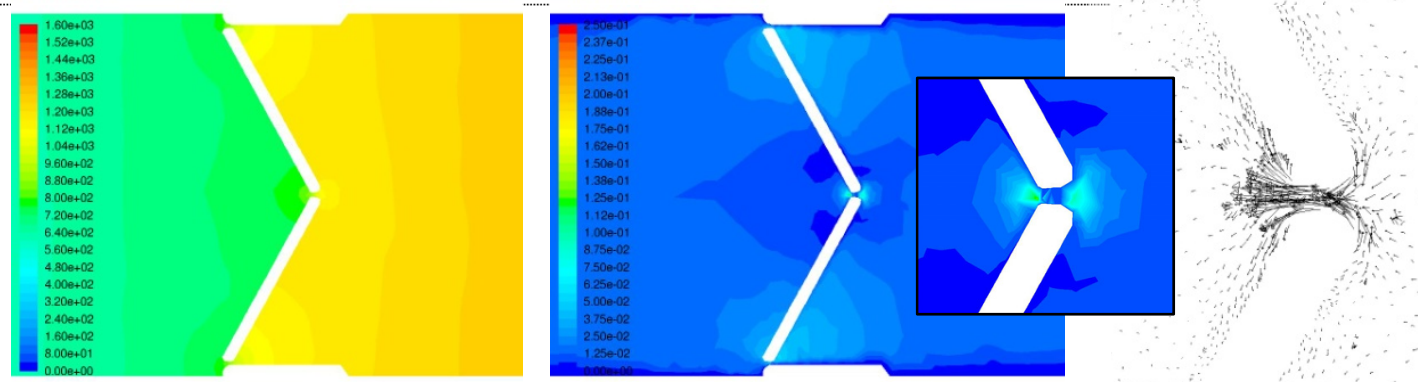

(c)
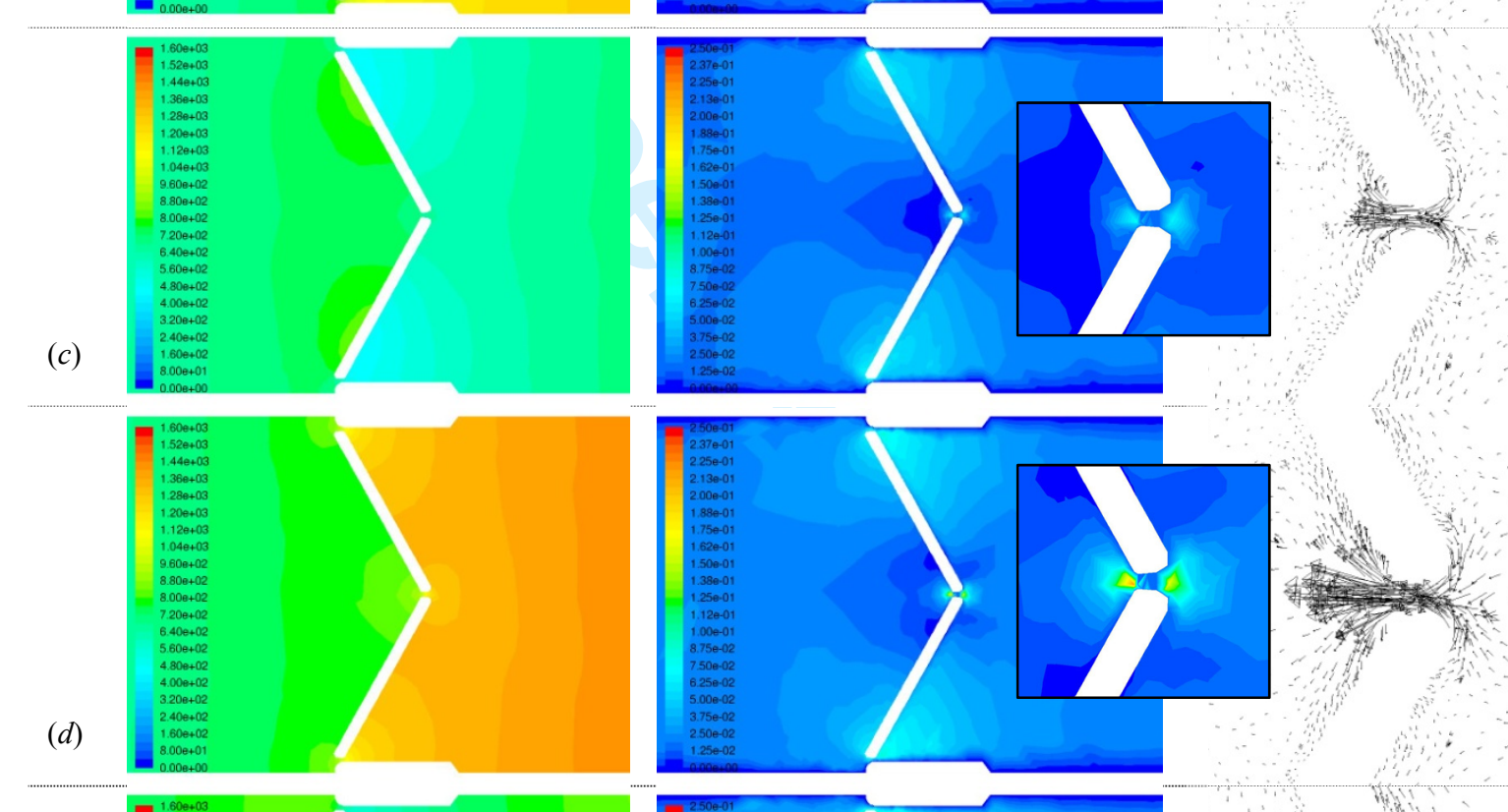

(e)
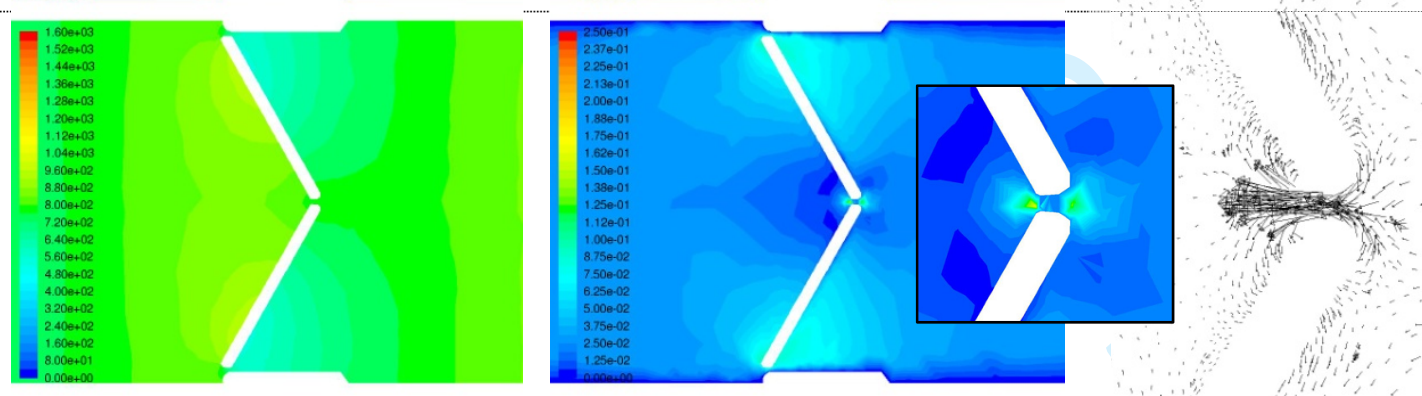

(f)
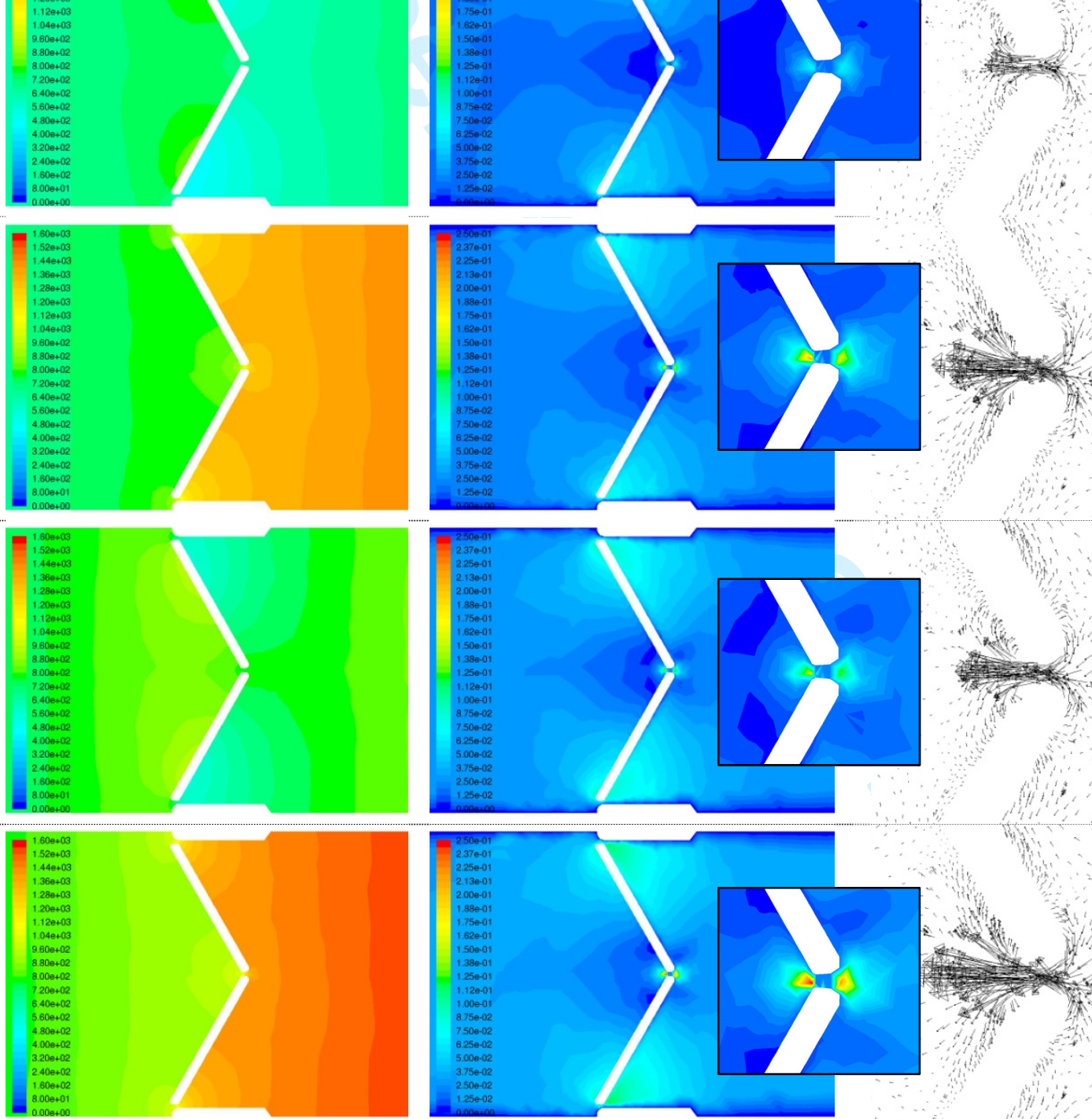

Figure 12. Plots of the pressure field in Pa (left), velocity field in $\mathrm{m} / \mathrm{s}$ (middle left), a zoom of the velocity field (middle right) and the velocity vectors at the b-datum gap $(r i g h t)$ for loose coupling $\left(\omega_{i}^{n+1}=0.325\right)$, visualized on a longitudinal section perpendicular to the leaflet rotation axes, taken at subsequent time steps $(t=0.004 \mathrm{~s}(a), t=0.0045 \mathrm{~s}(b), t=0.005 \mathrm{~s}(c), t=0.0055 \mathrm{~s}(d), t=0.006 \mathrm{~s}(e)$ and $t=0.0065 \mathrm{~s}(f))$. 
(a)
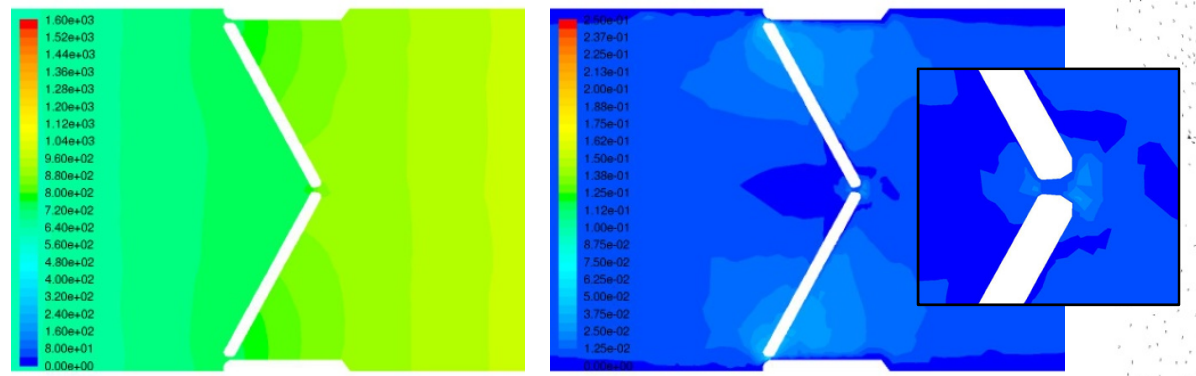

(b)
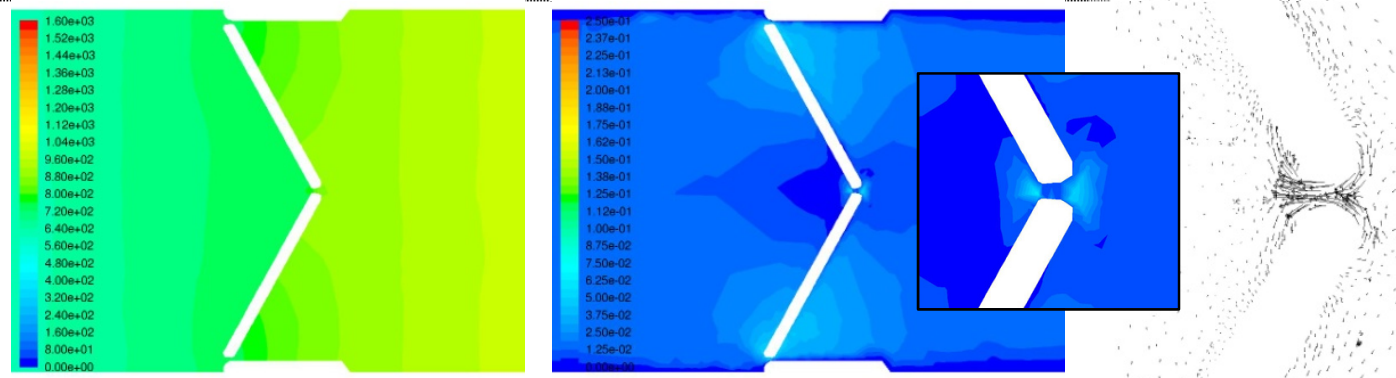

(c)
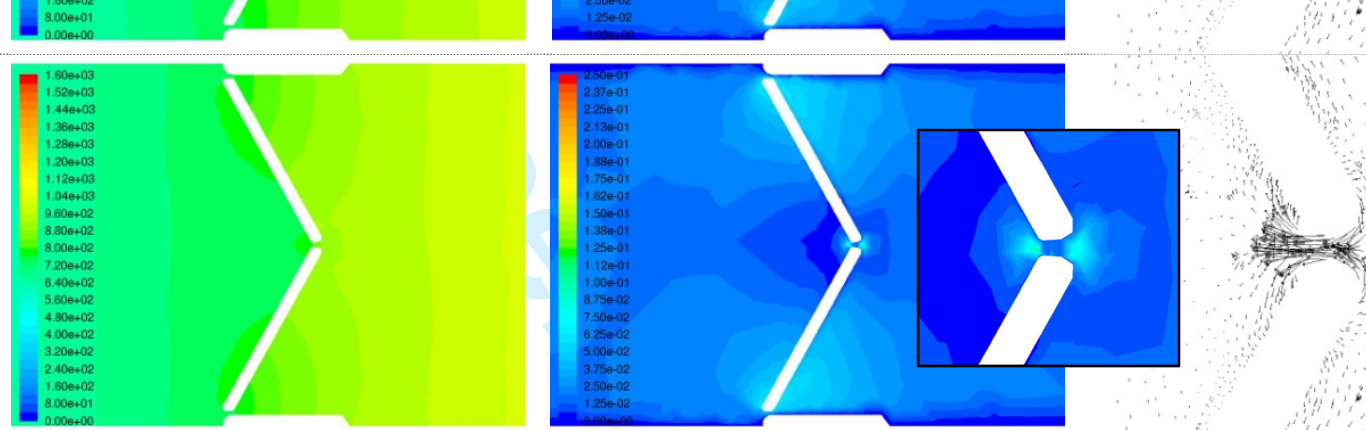

(d)
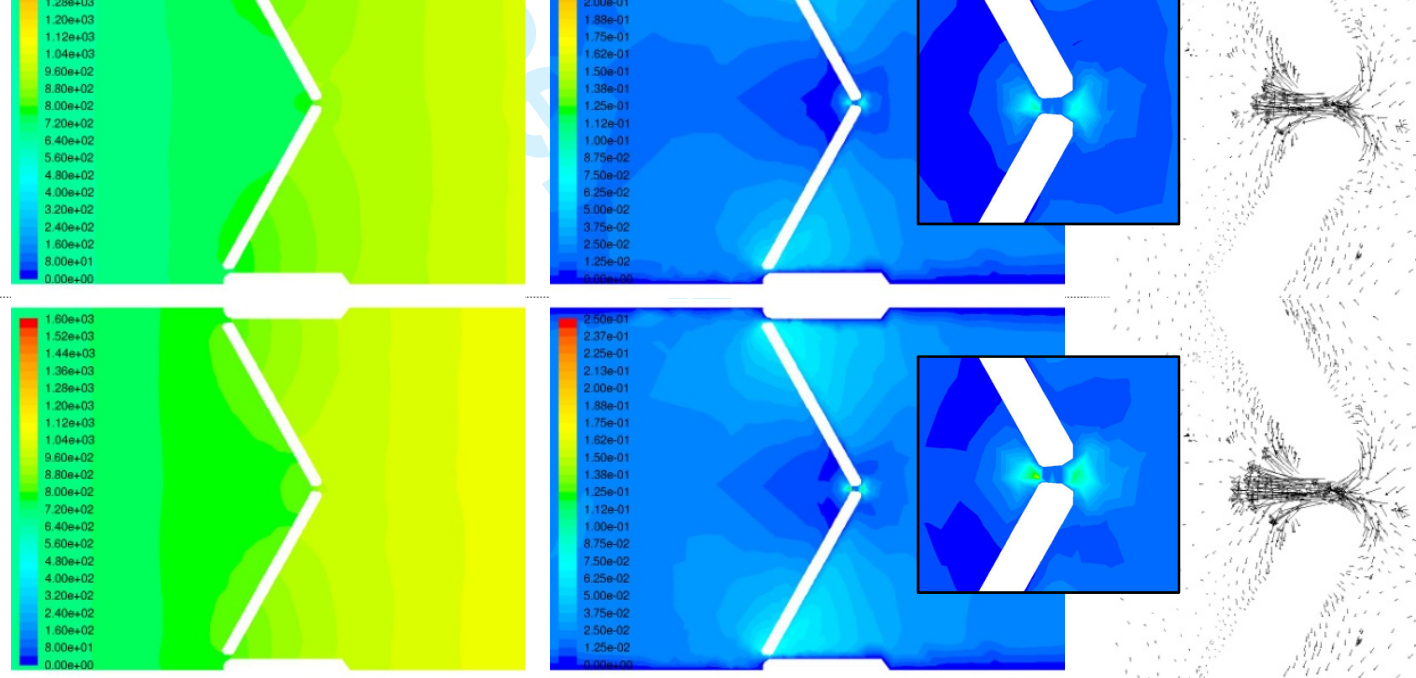

(e)
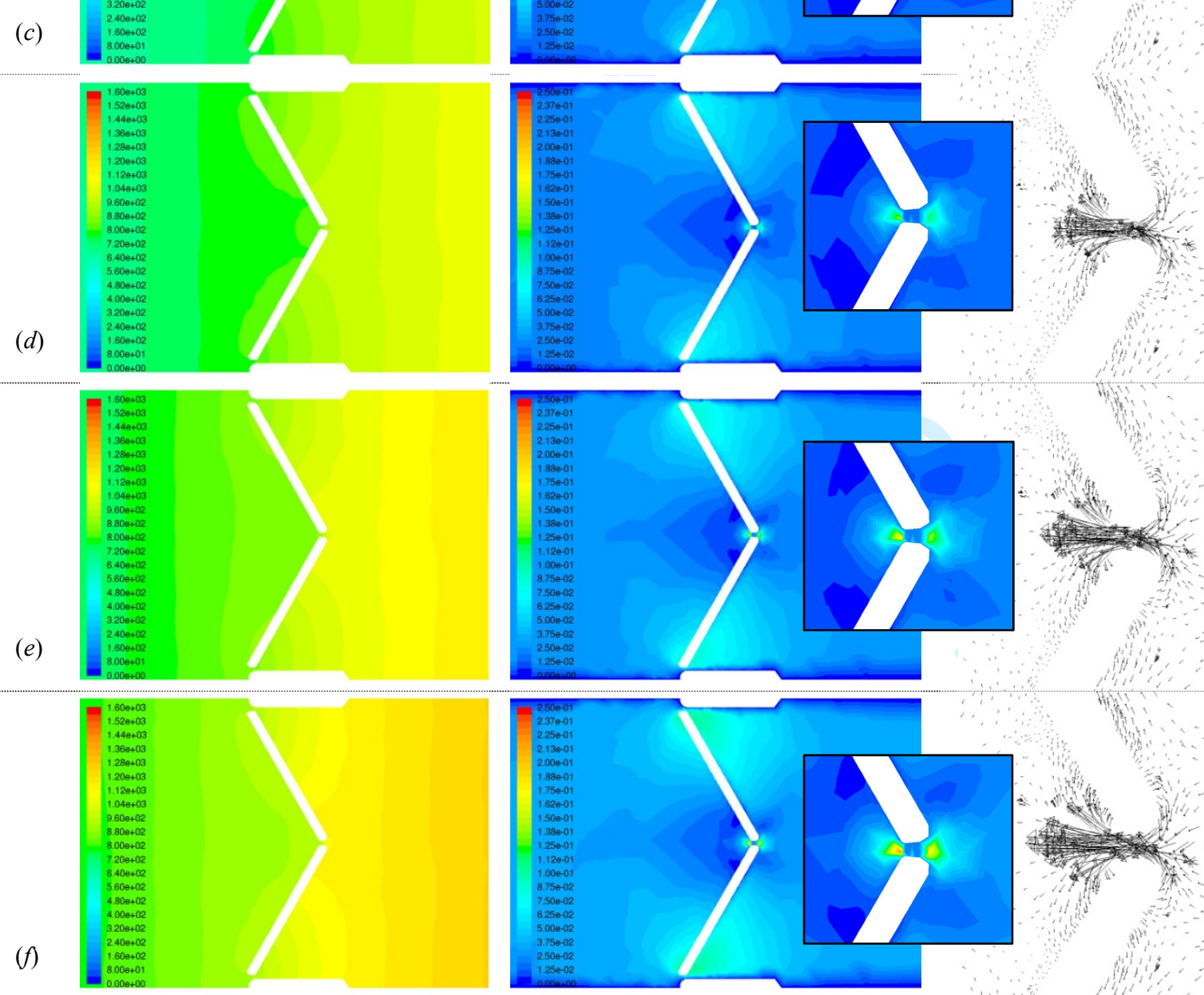

Figure 13. Plots of the pressure field in Pa (left), velocity field in $\mathrm{m} / \mathrm{s}$ (middle-left), zoom of the velocity field (middle-right) and the velocity vectors at the b-datum gap (right) for strong coupling (quasi-Newton), visualized on a longitudinal section perpendicular to the leaflet rotation axes, taken at subsequent time steps $(t=0.004 \mathrm{~s}(a), t=0.0045 \mathrm{~s}(b), t=0.005 \mathrm{~s}(c), t=0.0055 \mathrm{~s}(d), t=0.006 \mathrm{~s}(e)$ and $t=0.0065 \mathrm{~s}(f))$. 